\title{
SINGULAR BOHR-SOMMERFELD RULES FOR 2D INTEGRABLE SYSTEMS
}

\author{
By YVES COLIN DE VERDIÈRE AND SAN VŨ NGỌC
}

\begin{abstract}
This article gives Bohr-Sommerfeld rules for semiclassical completely integrable systems with two degrees of freedom with non-degenerate singularities (Morse-Bott singularities) under the assumption that the energy level of the first Hamiltonian is non-singular. The more singular case of focusfocus singularities was treated in previous works by San Vũ Ngọc. The case of one degree of freedom has been studied by Colin de Verdière and Parisse.

The results are applied to some famous examples: the geodesics of the ellipsoid, the $1: 2$-resonance, and Schrödinger operators on the sphere $S^{2}$. A numerical test shows that the semiclassical Bohr-Sommerfeld rules match very accurately the "purely quantum" computations.
\end{abstract}

๑) 2003 Éditions scientifiques et médicales Elsevier SAS

RÉSUMÉ. - Cet article fournit les règles de Bohr-Sommerfeld pour les systèmes complètement intégrables semi-classiques à deux degrés de liberté ayant des singularités non dégénérées (à la MorseBott), sous la condition que le niveau d'énergie du premier Hamiltonien soit non critique. Le cas plus singulier des singularités de type foyer-foyer a été traité dans des travaux antérieurs de San Vũ Ngọc. Le cas d'un seul degré de liberté a été étudié par Colin de Verdière et Parisse.

Les résultats sont appliqués à quelques exemples célèbres : les géodésiques de l'ellipsoïde, la résonance $1: 2$ et les opérateurs de Schrödinger sur la sphère $S^{2}$. Un test numérique montre que les règles de BohrSommerfeld semi-classiques sont en parfait accord avec les calculs "purement quantiques".

() 2003 Éditions scientifiques et médicales Elsevier SAS

\section{Introduction}

In this paper, we describe extensions of results of [10] to completely integrable semiclassical systems with 2 degrees of freedom. If $\widehat{H}_{1}, \widehat{H}_{2}$ are two commuting $h$-pseudodifferential operators on a 2-manifold $X$, we introduce the momentum map $F=\left(H_{1}, H_{2}\right): T^{\star} X \rightarrow \mathbb{R}^{2}$ where $H_{j}$ $(j=1,2)$ is the principal symbol of $\widehat{H}_{j}$; we assume that $F$ is a proper map. If $o=(0,0)$ is not a critical value of $H$, the existence and the construction of solutions of the system $\widehat{H}_{j} u=\mathrm{O}\left(h^{\infty}\right)$ has a long history and existence of solutions is well known to depend on the Bohr-Sommerfeld rules involving action integrals and Maslov indices of loops generating the homology of the fibre of $F$ which is a 2 -torus.

We will assume that $o$ is a critical value and more precisely that the critical points are of Morse-Bott type. A very simple classification of such points with the corresponding normal forms is given in [25]. The focus-focus case has been described in [27]. We will be interested in the case where the set of critical points of $H$ is a 2-dimensional manifold with a transversally hyperbolic (saddle) singularity: it means that 0 is not a critical value of $H_{1}$ and that $H_{2}$ 
restricted to Poincaré sections of the flow of $H_{1}$ admits critical points of saddle type. The set of critical values of $F$ is then a 1-dimensional submanifold of $\mathbb{R}^{2}$. The main result of our paper is a description of singular Bohr-Sommerfeld rules in this situation. These rules give necessary and sufficient conditions for existence of solutions of the system $\widehat{H}_{j} u=\mathrm{O}\left(h^{\infty}\right)$, $j=1,2$, and approximations of the solutions. More precisely, we show the existence near the singular fibre $\Lambda_{o}=F^{-1}(o)$ of a Hamiltonian $H_{p}$ with periodic flow which allows to reduce the classical study to the 1-dimensional case on the reduced phase space. This reduced phase space can be singular because the $S^{1}$ action induced by the flow of $H_{p}$ is not principal in general; nontrivial isotropy group isomorphic to $\mathbb{Z} / 2 \mathbb{Z}$ may appear. The possibility of this singularity and the fact that one is no longer working on a cotangent bundle make the semiclassical "reduction" more delicate.

We provide a description of $\Lambda_{o}$ : the topological type can be rather unusual like a Klein bottle!

The precise description of the quantisation rules is given in Theorem 2.7. There is one rule giving a quantum number associated to the periodic orbits of $H_{p}$ and another rule given in terms of the graph $G$ which is the quotient of $\Lambda_{o}$ by the $S^{1}$-action associated with $H_{p}$. In the spirit of [27], we interpret these rules as a universal regularisation of the usual Bohr-Sommerfeld rules for tori as these tori degenerate, leading to our main Theorems 2.19 and 2.20. These statements, in addition to proving the validity of the singular Bohr-Sommerfeld rules, allow us to have a description of the joint spectrum inside a fixed neighbourhood of $o$; however, some technical difficulties appear which are due to the possible non-connectedness of the fibres of $F$, especially in the $C^{\infty}$ category.

At the end we describe three examples for which we provide explicit calculation and numerical checking:

1. High energy limit for eigenvalues of the Laplacian on ellipsoids.

2. Semi-excited states for anharmonic oscillators with a resonance $2: 1$.

3. High energy limit for the Schrödinger spectrum on the 2 -sphere.

For the last two examples, numerical computations of eigenvalues of large matrices are compared with the eigenvalues obtained from the singular Bohr-Sommerfeld rules. We observe a very good accuracy of the results even for not very big quantum numbers.

\section{Classical mechanics}

The goal of this section is to give a description of the Lagrangian fibres of a 2-degrees-offreedom integrable system having non-degenerate rank-one singularities of hyperbolic type, in a neighbourhood of the critical fibre.

Let $(M, \omega)$ be a symplectic manifold of dimension 4 , and let $H_{1}, H_{2}$ be two Poisson commuting Hamiltonian functions in $C^{\infty}(M, \mathbb{R})$. The corresponding momentum map will be denoted by $F=\left(H_{1}, H_{2}\right)$; we shall always assume $F$ to be proper, $H_{1}$ to be non-singular on the level set $\Lambda_{o}:=F^{-1}(o)$ (for some point $o \in \mathbb{R}^{2}$ ). Moreover, we assume that $\Lambda_{o}$ is connected and that the critical points of $F$ on $\Lambda_{o}$ are transversally non-degenerate, in the following sense:

For any Poincaré section $\Sigma$ of the Hamiltonian flow of $H_{1}$, the restriction of $H_{2}$ to $\Sigma$ is a Morse function.

The main results of this section are

- The description of the topology of the fibre $\Lambda_{o}$ (Section 1.2).

- The construction of partial action-angle coordinates in a full neighbourhood of $\Lambda_{o}$; we show in particular that there exists a Hamiltonian $H_{p}$ defined in some neighbourhood of $\Lambda_{o}$ that 
Poisson commutes with $H_{j}, j=1,2$, and all orbits of which are periodic (Theorem 1.6). Moreover, up to finite covering, a neighbourhood of $\Lambda_{o}$ is symplectomorphic to a product of $T^{*} S^{1}$ by a "global" Poincaré section.

- The construction of normal forms for the system near each connected component of the critical set of $F$ (Theorem 1.13).

The topology of $\Lambda_{o}$ can in principle be obtained from Fomenko's description [14], and the first two points appear in Nguyên Tiên Dung [23]. However, we felt that these results deserved an independent description with detailed arguments.

\subsection{Notation}

For convenience of the reader we group here some of our notation: our integrable system is given by the momentum map $F=\left(H_{1}, H_{2}\right): M \rightarrow \mathbb{R}^{2}$. Because $F$ is proper, it is a momentum map for a Hamiltonian action of $\mathbb{R}^{2}$ on $M . \quad o=\left(a_{o}, b_{o}\right)$ is a critical value of $F, U$ small disk around $o$ in $\mathbb{R}^{2}$. The smooth energy level is $S_{o}=H_{1}^{-1}\left(a_{o}\right) . \Omega$ is $F^{-1}(U) ; \Lambda_{o}=F^{-1}(o)$. $\gamma=\bigcup_{i=1}^{N} \gamma_{i}$ is the critical set of $F$ in $\Lambda_{o} . \Gamma=\bigcup \Gamma_{i}$ is the critical set in $\Omega . \Lambda_{o} \backslash \gamma=\bigcup \Lambda_{\{i, j\}}^{k}$ where $\Lambda_{\{i, j\}}^{k}$ is a smooth Lagrangian cylinder whose closure is $\Lambda_{\{i, j\}}^{k} \bigcup \gamma_{i} \bigcup \gamma_{j}$. For any Hamiltonian $H_{\text {something }}$ we will denote by $\mathcal{X}_{\text {something }}$ the associated Hamiltonian vector field. $G$ is a graph with $N$ vertices associated to $\Lambda_{o}$. The Hessian of $H_{2}$ restricted to $\Sigma$ is $\mathcal{H}_{\Sigma}\left(H_{2}\right)$. The absolute value of its determinant (with respect to the density induced by the symplectic for on $\Sigma$ ) is independent on $\Sigma$ and denoted by $\left|\mathcal{H}_{\Sigma}\left(H_{2}\right)\right|$.

\subsection{Topology of $\Lambda_{o}$}

Proposition 1.1. - The critical set $\gamma$ of $F$ in $S_{o}$ is a compact submanifold of dimension 1 of $\Lambda_{o}$ which is a finite union of disjoint periodic orbits $\gamma_{i}(i=1, \ldots, N)$ of $\mathcal{X}_{1}$. The $\gamma_{i}$ admits orbit-cylinders $\Gamma_{i}$ which consists of $\gamma_{i}(a)$, a close to $a_{o}$.

Proof. - Locally the reduced manifold of $S_{o}:=\left\{H_{1}=a_{o}\right\}$ is symplectomorphic to a Poincaré section $\Sigma$ of $\mathcal{X}_{1}$. Because $\mathcal{X}_{1}$ is transversal to $\Sigma$ in $S_{o}$, a neighbourhood $V$ of $\Sigma$ in $S_{o}$ is diffeomorphic to $\Sigma \times I$, for a small interval $I$, in such a way that the trajectories of $\mathcal{X}_{1}$ are of the form $\{\sigma\} \times I, \sigma \in \Sigma$. Because $H_{2}$ is constant under the $\mathcal{X}_{1}$-flow, $H_{2}(\{\sigma\} \times I)=\left(H_{2}\right)_{\mid \Sigma}(\sigma)$. By hypothesis, $H_{2}$ restricted to $\Sigma$ admits a non-degenerated critical point at $\sigma:=\gamma \cap \Sigma$. Since the rank of $F$ is invariant by the flow of $\mathcal{X}_{1}$, we must have $\gamma \cap V=\{\sigma\} \times I$, which says that $\gamma$ is a smooth manifold of dimension 1. Because, by definition, $\gamma$ is closed in the compact set $\Lambda_{o}$ and hence is compact, it must be a union of circles $\gamma=\bigcup \gamma_{i}$. Then each of these circles must be an $\mathcal{X}_{1}$-orbit, and only a finite number $N$ of such critical circles arises due to the properness of $F$.

The non-degeneracy of $\left(H_{2}\right)_{\uparrow \Sigma}$ implies that the isolated critical point $\sigma=\sigma\left(a_{o}\right)$ depends smoothly on $a$ close to $a_{o}$. Therefore the above description extends to any leaf of the foliation $H_{1}=a, a$ close to $a_{o}$, yielding a smooth family of circles $\gamma_{i}(a)$. Since a small neighbourhood of $S_{o}$ in $M$ is diffeomorphic to $S_{o} \times \mathbb{R}$ such that $a$ is a coordinate for the second factor, the union $\Gamma_{i}=\bigcup_{a \text { close to } a_{o}} \gamma_{i}(a)$ is diffeomorphic to a cylinder $S^{1} \times\left(\mathbb{R}, a_{o}\right)$.

If the critical point $\sigma$ is a local maximum or minimum of $H_{2}, \Lambda_{o}$ reduces to one elliptic periodic orbit, a situation which was studied a long time ago by several people (see [7]). We will from now assume that this critical point is a saddle point. Then one can have several critical circles in $\gamma$; we show now how they can be connected to each other inside $\Lambda_{o}$.

PROPOSITION 1.2. $-\Lambda_{o} \backslash \gamma$ is a union of $\mathbb{R}^{2}$-orbits that are cylinders. Each cylinder contains in its closure 1 or $2 \gamma_{i}$ 's. We will denote by $\Lambda_{\{i, j\}}=\bigcup_{k}\left\{\Lambda_{\{i, j\}}^{k}\right\}$ the set of cylinders that connect $\gamma_{i}$ and $\gamma_{j}$. 


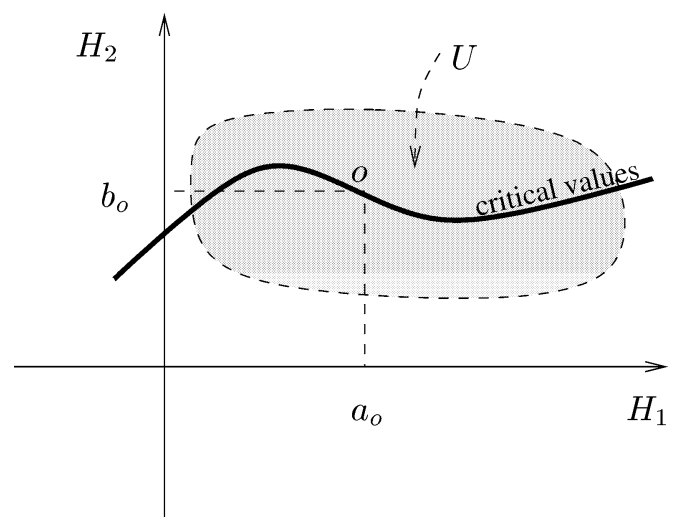

Fig. 1. Image of the momentum map. The set of critical values near $o$ is a smooth curve parameterised by $H_{1}$. If the critical point is a saddle point, the regular values are on both sides of the curve of critical values. If it is a maximum or a minimum, only one side is occupied.

Proof. - Since $\gamma$ is $\mathbb{R}^{2}$-invariant, $\Lambda_{o} \backslash \gamma$ is a union of orbits, on all of which the action is non-singular. These orbits are therefore 2 -dimensional quotients of $\mathbb{R}^{2}$, hence tori, cylinders or planes. Because $\Lambda_{o}$ is connected and contains singular points, any orbit contains critical points in its closure, which excludes tori. The local structure near each $\gamma_{i}$ will show independently the existence of periodic sub-orbits, leading to the non-triviality of the stabilisers of the $\mathbb{R}^{2}$-action, which excludes planes. Hence all orbits are cylinders.

Now, the closure of such a cylinder consists of singular orbits: there are 1 or 2 of them.

Definition 1.1.- We define the graph $G$ as follows: $G$ has $N$ vertices (where $N$ is the number of critical circles in $\Lambda_{o}$ ), and there are exactly $\left|\Lambda_{\{i, j\}}\right|$ different edges connecting the vertices $i$ and $j$.

Let $\Sigma$ be a Poincaré section at a point $m \in \gamma_{i}$. By hypothesis, $\Sigma \cap \Lambda_{o}$ is diffeomorphic to a "hyperbolic cross", the union of the local stable and unstable manifolds $W^{ \pm}(m)$ for the flow of $\mathcal{X}_{H_{2 \uparrow \Sigma}}$. Let $\Omega$ be a small neighbourhood of $\gamma_{i}$, and define $W^{ \pm}\left(\gamma_{i}\right)$ as the union of the connected components of $\left(\Lambda_{o} \backslash \gamma_{i}\right) \cap \Omega$ intersecting $W^{ \pm}(m)$. These manifolds do not depend on the choice of $m$.

PROPOSITION 1.3. - Either $W^{+}\left(\gamma_{i}\right)$ and $W^{-}\left(\gamma_{i}\right)$ are diffeomorphic to the disjoint union of 2 cylinders or both are diffeomorphic to 1 cylinder. In the first case the vertex $i$ of $G$ has degree 4 while in the second it has degree 2.

Proof. - The 2-manifold $\widetilde{W}^{+}\left(\gamma_{i}\right)=W^{+}\left(\gamma_{i}\right) \cup \gamma_{i}$ is a bundle on $\gamma_{i}$ whose fibre is an interval. There are exactly 2 possibilities up to diffeomorphism: the trivial and the Moebius bundle. In the first case, removing $\gamma_{i}$ gives 2 cylinders, while in the second it gives only 1 cylinder. Both bundles are isomorphic because the sum of their tangent bundle on $\gamma_{i}$ is a $\mathbb{R}^{2}$-bundle that is orientable as a symplectic bundle.

DEFINITION 1.2. - In the first case, $\gamma_{i}$ is called direct, in the second case, it is called reverse. 


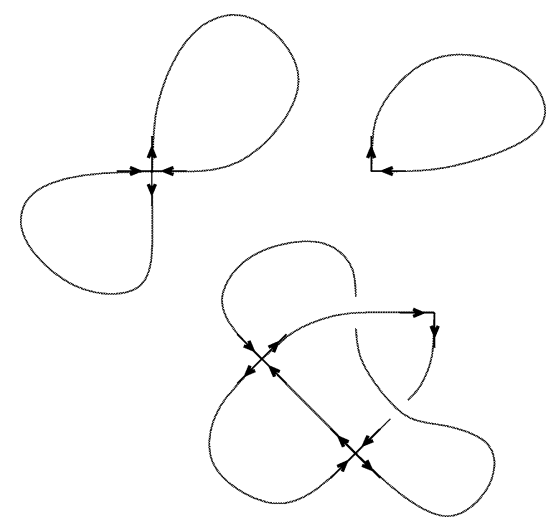

Fig. 2. The vertices of $G$ are of degree 2 or 4 .



a) direct case

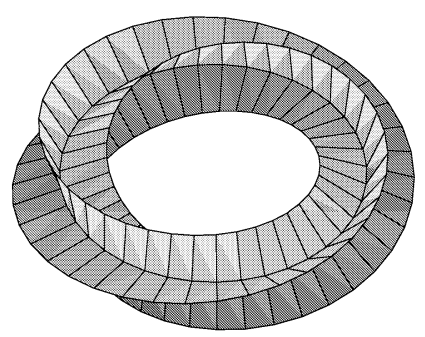

b) reverse case

Fig. 3. The neighbourhood of a critical circle.

\subsection{The classical commutant}

LEMMA 1.4. - For any smooth function $K$ commuting with $H_{1}$ and $H_{2}$, the vector field $\mathcal{X}_{K}$ can be uniquely written (in a neighbourhood of $\Lambda_{0}$ )

$$
\mathcal{X}_{K}=a \mathcal{X}_{1}+b \mathcal{X}_{2}
$$

for smooth functions $a$, $b$ commuting with $\mathrm{H}_{1}$ and $\mathrm{H}_{2}$.

Proof. - Near any non-singular point of $F$, apply the Darboux-Carathéodory theorem which makes $H_{1}=\xi$ and $H_{2}=\eta$ in a local symplectic coordinate chart $(x, y, \xi, \eta)$. Then $K=K(\xi, \eta)$, and the result follows.

Near a critical point in some $\gamma_{i}$, we use Theorem 1.5 below that reduces the situation to $H_{1}=\xi+a_{o}$ and $H_{2}=\Phi(\xi, y \eta)$, and hence to $\left(H_{1}=\xi, H_{2}=y \eta\right)$, for which we use the result of [25, Lemme 2.6] ([26, Lemme 2.2.7]).

THEOREM 1.5. - Around any point $m \in \gamma_{i}$, there exists a canonical chart $(x, y, \xi, \eta)$ in which

$$
\left\{\begin{array}{l}
H_{1}-a_{o}=\xi \\
H_{2}=\Phi(\xi, y \eta)
\end{array}\right.
$$

for some smooth function $\Phi$ defined near the origin, with

$$
\partial_{2} \Phi(0,0)>0
$$


Proof. - First construct a Darboux-Carathéodory chart for $H_{1}$, i.e. a system of symplectic coordinates $(x, y, \xi, \eta)$, with canonical form $\omega_{0}$, in which $\xi=H_{1}-a_{o}$ (this implies that $H_{2}$ is independent of $x$ ). In these coordinates, for any $x$, the plane $\{x\} \times\{0\} \times \mathbb{R}^{2}$ is a Poincare section for $H_{1}$, hence by hypothesis $(y, \eta) \rightarrow H_{2}(x, \xi, y, \eta)$ has, for each small $\xi$, a non-degenerate saddle point. The application of the Isochore Morse lemma [11] with parameter $\xi$ yields a local symplectomorphism $\phi_{\xi}(y, \eta)$ of the $(y, \eta)$-space, depending smoothly on $\xi$, such that

$$
H_{2}\left(x, \xi, \phi_{\xi}(y, \eta)\right)=\Phi(\xi, y \eta)
$$

for a function $\Phi$ with $\partial_{2} \Phi(0,0) \neq 0$. Applying the canonical transformation $(y, \eta) \rightarrow(-\eta, y)$ if necessary, one can assume that $\Phi$ satisfies condition (1) of the theorem. The map

$$
\phi:(x, \xi, y, \eta) \mapsto\left(x, \xi, \phi_{\xi}(y, \eta)\right)
$$

is a local diffeomorphism but need not be symplectic. A modification of $x$ shall solve the problem.

The 2-form $\omega_{1}:=\phi^{*}\left(\omega_{0}\right)$ splits as follows:

$$
\omega_{1}=\omega_{0}+d \xi \wedge \beta
$$

for some 1-form $\beta=\beta(\xi, y, \eta)$. From $d \omega_{j}=0$, one gets $d \xi \wedge d \beta=0$, which means $d_{(y, \eta)} \beta=0$. Hence there exists a smooth function $f(\xi, y, \eta)$ such that $d_{(y, \eta)} f=\beta$. Let $\phi_{1}$ be the diffeomorphism:

$$
\phi_{1}(x, \xi, y, \eta):=(x-f(\xi, y, \eta), \xi, y, \eta)
$$

so that

$$
\begin{aligned}
\phi_{1}^{*} \omega_{1} & =\phi_{1}^{*} \omega_{0}+\phi_{1}^{*}(d \xi \wedge \beta)=\phi_{1}^{*} \omega_{0}+d \xi \wedge \beta \\
& =\omega_{0}-d \xi \wedge d f+d \xi \wedge \beta=\omega_{0} .
\end{aligned}
$$

Thus $\phi \circ \phi_{1}$ is symplectic, and because it does not change $H_{1}=\xi-a_{o}$, it answers the question.

\subsection{A periodic Hamiltonian $H_{p}$ in $\Omega$}

The goal of this subsection is to prove the following Theorem 1.6 with the help of the three Lemmas 1.7, 1.8 and 1.9.

THEOREM 1.6 [23]. - There exists a unique (up to additive constant) Hamiltonian $H_{p}$ in $\Omega$ that fulfils the following conditions:

1. $H_{p}$ Poisson commutes with $H_{1}$ and $H_{2}$.

2. The flow of $H_{p}$ is $2 \pi$ periodic with minimal period $2 \pi$ outside $\Gamma$.

3. On $\Gamma_{i}$,

$$
\mathcal{X}_{p}=\alpha_{i}\left(H_{1}\right) \mathcal{X}_{1}
$$

with $\alpha_{i}$ a positive function.

Then the flow of $H_{p}$ is $2 \pi$-periodic on $\Gamma_{i}$ if the vertex $\{i\}$ is direct, and $\pi$-periodic in the reverse case.

Let us state now the following key lemmas:

$4^{\mathrm{e}}$ SÉRIE - TOME $36-2003-\mathrm{N}^{\circ} 1$ 
LEMMA 1.7. - Near each $\gamma_{i}$, there exists a unique (up to some additive constant if $a_{o} \neq 0$ ) Hamiltonian $H_{2, i}=H_{2}-\lambda_{i}\left(H_{1}\right) H_{1}$ which is critical on $\Gamma_{i}$.

Proof. - If $d H_{2}=f_{i}\left(H_{1}\right) d H_{1}$ on $\Gamma_{i}$, one gets the differential equation

$$
t \lambda_{i}^{\prime}+\lambda_{i}=f_{i}(t)
$$

which always admits a local solution.

DEFINITION 1.3. - A class of path $z \rightarrow\left[\gamma_{z}\right]$ (where [ ] means a homotopy or homology class) is called smooth if there exists locally a representative that smoothly depends on $z$.

LEMma 1.8. - Let us denote by $L_{z}$ the orbit of $z$ by the $\mathbb{R}^{2}$-action. There exists a unique mapping $z \rightarrow\left[\gamma_{z}\right]$ that is a smooth map from $\Omega$ into $H_{1}\left(L_{z}, \mathbb{Z}\right)$ and such that, if $z \in \gamma_{i}$, $\left[\gamma_{z}\right]=\nu\left[\gamma_{i}\right]$ where $\gamma_{i}$ is oriented according $\mathcal{X}_{1}$ and $\nu=1$ (resp. 2) if $\gamma_{i}$ is direct (resp. reverse).

Proof. - (a) The main point for this proof is to construct such a smooth family $\left[\gamma_{z}\right]$ near $\gamma_{i}$. By the Morse-Bott lemma (see Appendix 1.7) applied to the Hamiltonian $H_{2, i}$ of Lemma 1.7, there are a coordinate $x_{1}$ on the circle $\gamma_{i}=(\mathbb{R} / \mathbb{Z})$ and a fibre bundle $F_{ \pm}$of dimension 2 on $\gamma_{i}$ defined as quotient of the trivial bundle on $\mathbb{R}$ by identifying $\left(x_{1}, w\right) \in \gamma_{i} \times \mathbb{R}^{2}$ with $\left(x_{1}+1, \pm w\right)$ such that $H_{2, i}=x_{3} x_{4}$. We can assume that $\mathcal{X}_{1}$ has the same orientation as $\partial / \partial x_{1}$ on $\gamma_{i}$.

The above Morse-Bott lemma can be applied with the parameter $H_{1}$. Therefore, one gets coordinates $\left(x_{1}, x_{2}, x_{3}, x_{4}\right)$ on a full neighbourhood of $\gamma_{i}$ in $M$ by letting $x_{2}=H_{1}$.

We choose then $\gamma_{z}$ to be the path given in these coordinates by $t \rightarrow\left(t, x_{2}, x_{3}, x_{4}\right)$ with $t \in \mathbb{R} / \mathbb{Z}$ (direct case) and $t \in \mathbb{R} / 2 \mathbb{Z}$ (reverse case). These paths are drawn on the leaf $\left(H_{1}, H_{2, i}\right)=$ const, and hence on a leaf of $F=\left(H_{1}, H_{2}\right)$. The last assumption of Lemma 1.8 follows from Proposition 1.3.

(b) Far from $\gamma_{i}$, we construct $\gamma_{z}$ on $\Lambda_{o}$ by taking the cylinder equator with the orientation which in the affine structure of $\Lambda_{\{i, j\}}^{k}$ is given by projecting $\mathcal{X}_{1}$. Then we extend to the nearby Lagrangian leaves by local triviality of the foliation by orbits. Because these $\gamma_{z}$ are homotopic to the ones constructed in a) and lying on the same Lagrangian leaf, it is easy to realise this homotopy as an isotopy, thus yielding a smooth family of loops in $\Omega$.

(c) It remains now to use these $\gamma_{z}$ in order to define the action variable.

LEMMA 1.9. - The symplectic form $\omega$ is exact in $\Omega$, i.e. there exists $\alpha, 1$-form in $\Omega$ with $d \alpha=\omega$.

Proof. - The set $\Lambda_{o}$ is Lagrangian and any 2-cycle can be deformed inside $\Lambda_{o}$.

Proof of Theorem 1.6. - Put $H_{p}(z)=(1 / 2 \pi) \int_{\gamma_{z}} \alpha$. $H_{p}$ is smooth and commutes with $H_{1}$ and $H_{2}$ (because it is constant on $\mathbb{R}^{2}$-orbits). Moreover on $\mathbb{R}^{2}$-orbits that are tori the orbits of $\mathcal{X}_{p}$ are $2 \pi$-periodic with orbits homotopic to $\gamma_{z}$ (by usual action-angle coordinates). On the $\gamma_{i}$ 's, the period is $2 \pi$ in the direct case and $\pi$ in the reverse case.

The affine structure on the Lagrangian cylinders $\Lambda_{\{i, j\}}^{k}$ and condition (3) imply the uniqueness of $\mathcal{X}_{p}$ on $\Lambda_{o}$. Now suppose $H_{p}^{\prime}$ is another Hamiltonian with the same properties, and let $z$ be a point in $\Lambda_{o} \backslash \gamma$. Since the orbits under $\mathcal{X}_{p}$ and $\mathcal{X}_{p}^{\prime}$ of $z$ are equal, the orbits of points near $z$ in a same level set of $F$ (different from $\Lambda_{o}$ ) are homotopic. But these level sets are Liouville tori for which we know that $\mathcal{X}_{p}$ and $\mathcal{X}_{p}^{\prime}$ must be equal.

Remark 1.1. - Step (a) of the proof does not use the nature of $\Lambda_{\{i, j\}}^{k}$. Therefore, (a) + (c) gives a Hamiltonian $H_{p}$ verifying the conditions of Theorem 1.6 but in a neighbourhood of $\gamma_{i}$ 
only. This suffices to prove that the $\mathbb{R}^{2}$-action has non-trivial stabilisers, whence $\Lambda_{\{i, j\}}^{k}$ must be cylinders, thus finishing the proof of Proposition 1.2.

\section{5. $S^{1}$ reduction}

The flow of $\mathcal{X}_{p}$ yields a locally free Hamiltonian action of $S^{1}$ on $\Omega$, which is free outside $\Gamma$. The goal of this subsection is to complete the geometric description of our singular fibration with the help of this action. It is useful for the understanding of Section 2, but since our semiclassical framework will be based upon standard pseudodifferential quantisation, which requires cotangent bundles as phase spaces, some results of this section stand on their own and will not have (here) any semiclassical analogue. Note that our proof of the reduction Theorem 1.11 is new and will be used again for Theorem 1.13 (which will be quantised).

Let $c_{o}=H_{p}(m)=H_{p}\left(\Lambda_{o}\right)$. We denote by $W$ the reduced space

$$
W=H_{p}^{-1}\left(c_{o}\right) \cap \Omega / S^{1} .
$$

$W$ is a symplectic orbifold (see e.g. [3]). It is a smooth manifold if and only if the action is free, that is if and only if no vertex of reverse type are present in the graph $G$. Otherwise, it has singularities at the critical orbits $\gamma_{i}$. Since these critical orbits come in families depending on the value of $H_{1}$, yielding local orbit cylinders, and because $d H_{p}(m)=\lambda d H_{1}(m), \lambda \neq 0$, only one orbit of each local cylinder meets $H_{p}^{-1}\left(c_{o}\right)$, ensuring that the critical orbits give isolated singularities in $W$.

Let

$$
H_{q}=-b\left(H_{1}-a_{o}\right)+a\left(H_{2}-b_{o}\right),
$$

where $a>0$ and $b$ are the real constants such that, on $\Lambda_{o}, \mathcal{X}_{p}=a \mathcal{X}_{1}+b \mathcal{X}_{2}$ (cf. Lemma 1.4). Then $\Lambda_{o}=H_{p}^{-1}\left(c_{o}\right) \cap H_{q}^{-1}(0)$. (This still holds of course for a generic choice of $(a, b)$.) Since $H_{q}$ is $S^{1}$-invariant, it defines a smooth Hamiltonian function $\widetilde{H}_{q}$ on $W$. The graph $G$ can be viewed as the quotient of $\Lambda_{o}$ by $S^{1}$, and thus is identified to the level set $\widetilde{H}_{q}^{-1}(0)$.

Proposition 1.10. - If the $S^{1}$-action is free (i.e. all vertices of $G$ of degree 4), then a neighbourhood $\Omega$ of $\Lambda_{o}$ in $M$ is diffeomorphic to the direct product $S^{1} \times \mathbb{R} \times W$ (hence $\Lambda_{o}$ is diffeomorphic to the direct product $S^{1} \times G$ - these diffeomorphisms are equivariant with respect to the natural action of $S^{1}$ on itself).

Remark 1.2. - In this case, $W$ can be regarded as a "global" Poincaré section for $\mathcal{X}_{p}$.

Proof. - We choose now $\Omega$ to be of the form $\Omega_{o} \times I$ where $I$ is some open interval around $0, \Omega_{o}$ is a small invariant neighbourhood of $\Lambda_{o}$ in $H_{p}^{-1}\left(c_{o}\right)$, and $H_{p}\left(\Omega_{o} \times\{\xi\}\right)-c_{o}=\xi$. If the action is free, $\Omega_{o}$ is a principal $S^{1}$-bundle over $W$. It is topologically classified by its holonomy class in $H^{1}\left(W, S_{\mathfrak{d}}^{1}\right)$, where $S_{\mathfrak{d}}^{1}$ is the sheaf of germs of smooth functions on $W$ with values in $S^{1}$ (see [19]). Using the short exact sequence $0 \rightarrow \mathbb{Z} \rightarrow \mathbb{R} \rightarrow S^{1} \rightarrow 0$, and the fact that the sheaf $\mathbb{R}_{\mathfrak{d}}$ is fine, one gets an isomorphism

$$
H^{1}\left(W, S_{\mathfrak{d}}^{1}\right) \simeq H^{2}(W, \mathbb{Z})
$$

yielding the so-called Chern class of the bundle. But $W$ retracts onto $G$ and $G$ is 1-dimensional, so $H^{2}(W, \mathbb{Z})=0$, and $\Omega_{o}$ is a trivial bundle. 
THEOREM 1.11 [23]. - If the $S^{1}$-action is free, then $(\Omega, \omega)$ is symplectomorphic to a neighbourhood of $S^{1} \times\{0\} \times W$ in

$$
\left(T^{*} S^{1} \times W, d \xi \wedge d x+\pi^{*} \omega_{W}\right)
$$

with $H_{p}-c_{o}=\xi$. Here $\pi$ is the projection onto $W$ and $\omega_{W}$ is the symplectic form of $W$.

Proof. - First apply Proposition 1.10 and let $\xi=H_{p}-c_{o}$ be a coordinate for the $\mathbb{R}$ factor. Then choose the conjugate angle variable $x$ (pick up some coordinate $\theta$ in $S^{1}$, an origin $\theta_{0}$, and let $x(\theta)$ be the time required to go from $\theta_{0}$ to $\theta$ under the Hamiltonian action of $\xi$ ), so that $\{\xi, x\}=1$. Because $\omega$ is $S^{1}$-invariant, it does not depend on $x$; using the equivariant Darboux-Weinstein theorem [28], one can assume that $\omega=\omega_{\mid \xi=0}$, and so does not depend on $\xi$ either.

Because for any $\xi, \mathcal{X}_{p}$ is $\omega$-orthogonal to $S^{1} \times\{\xi\} \times W$, one easily checks by taking local coordinates on $W$ that

$$
\omega=d \xi \wedge d x+d \xi \wedge \pi^{*} \beta+\pi^{*} \omega_{W},
$$

where $\beta$ is a one-form on $W$, and $\omega_{W}$ a non-degenerate 2 -form on $W$. The closedness of $\omega$ (and its independence on $\xi$ ) implies $d \omega_{W}=0$ and $d \beta=0$, the latter yielding $d\left(\xi \pi^{*} \beta\right)=d \xi \wedge \pi^{*} \beta$. Let us now apply Moser's path method to get rid of this term. We let

$$
\omega_{t}:=d \xi \wedge d x+\pi^{*} \omega_{W}+t d\left(\xi \pi^{*} \beta\right)
$$

and wish to construct an isotopy $\varphi_{t}$ of diffeomorphisms of $\Omega$ such that $\varphi_{t}^{*} \omega_{t}=\omega_{0} . \varphi_{t}$ is then given as the flow of the vector field $X_{t}$ defined by $i_{X_{t}} \omega_{t}+\xi \pi^{*} \beta=0$. It is easy to check that $\omega_{t}$ is non-degenerate for all $t$ so that $X_{t}$ is uniquely defined. Moreover, because of its defining equation, $X_{t}$ is of the form $\xi \iota_{*} Y_{t}\left(\iota\right.$ is the inclusion $\left.W \hookrightarrow T^{*} S^{1} \times W\right)$, where $Y_{t}$ is a vector field on $W$ satisfying $i_{Y_{t}}\left(\omega_{t}\right)_{\uparrow W}+\beta=0$. Therefore $\varphi_{t}$ is of the form

$$
(x, \xi, w) \rightarrow\left(x, \xi, \phi_{\xi t}(w)\right)
$$

(where $\phi_{t}$ is the flow of $Y_{t}$ ) and hence preserves $x$ and $\xi$. If $Y_{t}$ can be integrated up to the time $t_{0}>0$, then $X_{t}$ can be integrated up to the time 1 for $\xi \leqslant t_{0}$. The diffeomorphism $\varphi_{1}$ then answers the question.

Remark 1.3. - The formula $\omega=d \xi \wedge d x+\pi^{*} \omega_{W}$ ensures that $\omega_{W}$ is the natural symplectic form on $W$ obtained by the reduction process.

THEOREM 1.12. - In the general case, there exists a smooth double covering $\Omega^{*}$ of $\Omega$ in which the action is free. The reduced manifold $W^{*}$ is a covering of $W$ that is ramified of degree 2 at the critical orbits $\gamma_{i}$.

Proof. - Choose $\Omega$ to be a relatively compact invariant neighbourhood of $\Lambda_{o}$ in $M$. Then $H_{p}^{-1}\left(c_{o}\right) \cap \Omega$ has a smooth non-empty invariant boundary, and because this boundary does not meet any critical orbit, the closure $\bar{W}=\overline{H_{p}^{-1}\left(c_{o}\right) \cap \Omega} / S^{1}$ is a relatively compact surface with a non-empty smooth boundary. Let $p_{i}, i=1, \ldots, \ell$, be the images under reduction of the critical circles $\gamma_{i}$ and let $\check{W}$ be the surface $\bar{W}$ after removal of small disks $D_{i}$ around each $p_{i}$. It is still a smooth surface with boundary, whose fundamental group is free [1], and generated by some $\mu_{1}, \ldots, \mu_{k}, \delta_{1}, \ldots, \delta_{\ell}$, with $\delta_{i}=\partial D_{i}$. Let $\mathcal{D} \subset \pi_{1}(\check{W})$ be the free subgroup generated by $\mu_{1}, \ldots, \mu_{k}, \delta_{1}^{2}, \ldots, \delta_{\ell}^{2}$, and let $\breve{W}^{*}$ be the corresponding smooth covering of $\check{W}$. Gluing back the disks $D_{i}$ defines a covering space $W^{*}$ of $W$ that is ramified of degree two at each $p_{i}$. Define now 
$\Omega^{*} \subset \Omega \times W^{*}$ such that the following diagram commutes:

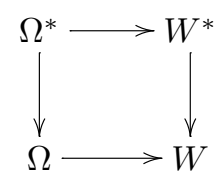

Since the local structure near $\gamma_{i}$ (see Theorem 1.13 in the next section) gives a model for the covering $\Omega^{*} \rightarrow \Omega, \Omega^{*}$ is naturally endowed with a smooth structure compatible with that of $\Omega$. The lifts of $\gamma_{i}$ are critical circles in $\Omega^{*}$ that become of direct type. As a result, all critical circles in $\Omega^{*}$ are of direct type, which means that the lifted $S^{1}$ action is free.

\subsection{Normal forms near $\gamma_{i}$}

Choose any Hamiltonian function $H_{q}$ near $\gamma_{i}$ that commutes with $H_{1}$ and $H_{2}$ and such that, for any $o$ in the local curve of critical values of $F$,

- $H_{q}\left(\Lambda_{o}\right)=0$;

- on $\Lambda_{o} \backslash \gamma_{i}, \mathcal{X}_{p}$ and $\mathcal{X}_{q}$ are linearly independent, and the automorphism

$$
\left(\mathcal{X}_{1}, \mathcal{X}_{2}\right) \rightarrow\left(\mathcal{X}_{p}, \mathcal{X}_{q}\right)
$$

is orientation preserving.

For instance, the previously defined $H_{q}$ (Eq. (7)) is a good choice, which is independent on $i$, but any generic linear combination $H_{q}=\alpha\left(H_{1}-a_{o}\right)+\beta\left(H_{2}-b_{o}\right), \beta>0$ would also do.

Then the following theorem states a simple normal form near $\gamma_{i}$ for the new system $\left(H_{p}, H_{q}\right)$. It will be the main tool for the semiclassical analysis, for it reduces the situation to the case of a cotangent bundle.

THEOREM 1.13. - There exist coordinates $(x, y)$ on $R=\mathbb{R} \times] A, B[$ that give local coordinates on $\widetilde{W}^{+}\left(\gamma_{i}\right)$ by taking the quotient by

$$
(x, y) \rightarrow(x+2 \pi, y)
$$

in the direct case and

$$
(x, y) \rightarrow(x+\pi,-y)
$$

in the reverse case and a canonical diffeomorphism of a neighbourhood of $\gamma_{i}$ into a neighbourhood of the " $\xi=c_{o}$-section" of $T^{*}\left(\widetilde{W}^{+}\left(\gamma_{i}\right)\right.$ ) (recall that $c_{o}=H_{p}\left(\Lambda_{o}\right)$ ) such that with respect to canonical coordinates we get

$$
\left\{\begin{array}{l}
H_{p}=\xi \\
H_{q}=\Phi(\xi, y \eta),
\end{array}\right.
$$

for some smooth function $\Phi$ defined near the origin, with $\partial_{2} \Phi(0,0)>0$.

Proof. -1 . We first wish to prove that the restriction of $H_{q}$ to the locally reduced manifold $W:=H_{p}^{-1}\left(c_{o}\right) / \exp t \mathcal{X}_{p}$ has a non-degenerate saddle point. Let $m \in \gamma_{i}$ and let $(s, \sigma, u, v)$ be local coordinates near $m$ such that $\sigma=H_{1}-a_{o}$ and the flow of $\mathcal{X}_{1}$ is just translation on the $s$ variable. Then because $d H_{p}(m)=\lambda d H_{1}(m)$ with $\lambda \neq 0$, the map

$$
(s, \sigma, u, v) \rightarrow\left(s, H_{p}-c_{o}, u, v\right)
$$


is a local diffeomorphism of $\left(\mathbb{R}^{4}, 0\right)$ that sends $(s, 0,0,0)$ to $(s, 0,0,0)$. Therefore one can take $(u, v)$ as local coordinates for $W$, and we wish to prove that $\left(H_{q}\right)_{\uparrow W}(u, v)$ has a non-degenerate saddle point at $(0,0)$.

Let $\mathcal{M}=\left(\begin{array}{ll}a & b \\ c & d\end{array}\right)$ be the matrix of smooth functions such that

$$
\left(d H_{1}, d H_{2}\right)=\mathcal{M} \cdot\left(d H_{p}, d H_{q}\right) .
$$

Since $H_{2}$ is of the form $H_{2}=F\left(H_{1}, u, v\right)$, one has

$$
d H_{2}=K \cdot d H_{1}+A,
$$

where $K=\partial_{1} F$ and $A=A\left(H_{1}, u, v\right)$ is a one-form on $\{0\} \times\{0\} \times \mathbb{R}^{2}$ depending smoothly on $H_{1}$, that vanishes at $m$ and whose differential at $m$ is a non-degenerate quadratic form of hyperbolic type. Using $\mathcal{M}$ one gets

$$
(c-K a) d H_{p}=(K b-d) d H_{q}+A .
$$

The claim is that $(K b-d)(m) \neq 0$. Indeed, $(c-K a)$ and $(K b-d)$ cannot simultaneously vanish because $\mathcal{M}$ is invertible. It suffices then to see that $A(m)=0$ whereas by hypothesis $d H_{p}(m) \neq 0$.

Therefore, we have, on $T W \subset \operatorname{ker} d H_{p}$,

$$
d\left(H_{q}\right)_{\uparrow T W}=-\frac{1}{K b-d} A
$$

which implies that $d\left(H_{q}\right)_{\uparrow T W}$ possesses, as $A$ does, a non-degenerate differential of hyperbolic type.

2. Now, we consider a neighbourhood of the whole critical circle $\gamma_{i}$ and use Weinstein's theorem with $S^{1}$ action to reduce to the cotangent space of $\widetilde{W}^{+}\left(\gamma_{i}\right)$ with $\left(H_{p}-c_{o}=\xi\right.$, $\left.H_{q}=H_{q}(\xi, y, \eta)\right)$. In these coordinates (we consider first the direct case), $W$ is naturally identified with $\{0\} \times\{0\} \times \mathbb{R}^{2}$, so the previous point shows that $(y, \eta) \rightarrow H_{q}(\xi, y, \eta)$ has, for each small $\xi$, a non-degenerate saddle point. Then the proof goes exactly as that of Theorem 1.5. Eqs. (2)-(5) are valid if $H_{1}$ and $H_{2}$ are replaced by $H_{p}$ and $H_{q}$, and $\omega_{0}$ is the canonical 2-form of $T^{*}\left(\widetilde{W}^{+}\left(\gamma_{i}\right)\right)$.

In the reverse case, the proof is the same but we need the Isochore Morse lemma for functions that are invariant by the involution $\sigma(x)=-x$ : it it then possible to choose the diffeomorphism $F$ commuting with $\sigma$. This fact follows easily from the proof given in [11]: in the lemme principal p. 283, we choose $\eta$ such that $\sigma^{\star}(\eta)=-\eta$. It implies that $X_{t}$ commutes with the involution.

Remark 1.4. - We decide to give to the graph $G$ the orientation of the flow of $H_{q}$ defined by (7). Near a vertex $\gamma_{j}$, it is also given by the flow of the normal form $y \eta$.

\subsection{Appendix: Morse-Bott lemma}

DEFINITION 1.4. - Let $f: X \rightarrow \mathbb{R}$ be a smooth function. A submanifold $W$ of $X$ is called a Morse-Bott critical manifold if every point $w \in W$ is a critical point of $f$ and if the restriction of $f^{\prime \prime}(w)$ to the normal bundle $T_{w} X / T_{w} W$ is non-degenerate.

Morse-Bott critical manifold arises in many situations especially when $f$ is invariant by a Lie group action. An extension of the Morse lemma is available in that case. In some situations, 
there is global topological problem with the subbundles $N_{ \pm}$of the normal bundle generated by eigenspaces of $f^{\prime \prime}$ associated with $>0$ (resp. $<0$ ) eigenvalues.

LemMa 1.14 (Morse-Bott lemma).-Assume we have a Morse-Bott connected critical manifold $W$ for a function $f: X \rightarrow \mathbb{R}$. Let $N$ be the normal bundle of $W$ and $F$ the Hessian of $f$ which is a non-degenerate quadratic form on $N$. Then there exists a diffeomorphism of a neighbourhood of $W$ on a neighbourhood of the 0 -section in $N$ which conjugates $f$ to $F+c$.

If $W$ is connected, a complete set of invariant of $f$, up to smooth conjugacy near $W$, is given by the pair $\left(N_{+}, N_{-}\right)$of bundles on $W$ up to isomorphism.

\section{Semiclassical analysis}

The aim of this section is to express the singular Bohr-Sommerfeld quantisation rules for quantum integrable systems whose classical counterpart fulfils the hypothesis of the previous section.

Let $X$ be a 2-dimensional differential manifold, and let $\widehat{H}_{1}(h), \widehat{H}_{2}(h)$ be commuting $h$ pseudodifferential operators, with real principal symbols $H_{1}, H_{2}$. Assume that the momentum map $F=\left(H_{1}, H_{2}\right)$ satisfies the hypothesis of Section 1. In all of this section, the 1-form $\alpha$ of Lemma 1.9 is taken to be the canonical Liouville 1-form of the cotangent bundle $T^{*} X$. Then $H_{p}$ (Theorem 1.6) is uniquely defined as the action integral with respect to $\alpha$. For any $E$ in the image of $F$, the sub-principal form $\kappa_{E}$ is the closed differentiable 1-form on $\Lambda_{E}:=F^{-1}(E)$ defined at its regular points by $\kappa_{E}\left(\mathcal{X}_{j}\right)=-r_{j}$, where $r_{j}$ is the sub-principal symbol of $\widehat{H}_{j}$.

\subsection{The microlocal normal form}

We will prove here a semiclassical analogue of Theorem 1.13, which was particularly fit for this purpose since it reduced the situation to that of a cotangent bundle, for which the usual pseudodifferential quantisation can be used. A semiclassical analogue of Theorem 1.11 should also be interesting, but would involve symplectically reduced cotangent bundles, for which Toeplitz quantisation is needed, a theory that we do not want to enter here.

In this section a critical circle $\gamma_{j} \subset \Lambda_{o}$ is fixed. Theorem 1.13 identifies, via a symplectomorphism $\psi$, a neighbourhood of $\gamma_{j}$ in $T^{*} X$ with a neighbourhood of the zero section of a cotangent bundle of the form $T^{*}\left(\mathbb{R}^{2} / \sigma\right)$, where $\sigma(x, y)=(x+2 \pi, y)$ in the direct case, and $\sigma(x, y)=(x+\pi,-y)$ in the reverse case.

It is easy to check that Weyl quantisation satisfies, for a symbol $a \in C_{0}^{\infty}\left(\mathbb{R}^{2}\right)$ :

$$
\sigma^{*} O p_{h}^{W}(a)=O p_{h}^{W}\left(a \circ T^{*} \sigma\right) \sigma^{*},
$$

where $\sigma^{*}$ is the adjoint operator $u \mapsto u \circ \sigma$, and $T^{*} \sigma$ is the cotangent lift of $\sigma$. Therefore, if $a=a \circ T^{*} \sigma$, then $O p_{h}^{W}(a)$ acts on the space of functions $u$ that are invariant under $\sigma: u \circ \sigma=u$, which is the space of functions defined on a cylinder in the direct case, and on the Moebius strip in the reverse case. In particular, $Q_{1}(h)=O p_{h}^{W}(\xi)$ and $Q_{2}(h)=O p_{h}^{W}(y \eta)$ are well-defined differential operators on $\mathbb{R}^{2} / \sigma$ :

$$
Q_{1}(h)=\frac{h}{i} \frac{\partial}{\partial x}, \quad Q_{2}(h)=\frac{h}{i}\left(y \frac{\partial}{\partial y}+\frac{1}{2}\right) .
$$

Let $\Psi^{0}$ be the algebra of operators of the form $O p_{h}^{W}(a(h))$ for classical symbols $a(h)$ on $T^{*}\left(\mathbb{R}^{2} / \sigma\right)$, modulo those whose symbol is $\mathrm{O}\left(h^{\infty}\right)$. Before stating the result of this section, we introduce the following spaces: 
Definition 2.1. - The classical and semiclassical commutants $\mathfrak{C}_{c l}\left(\gamma_{j}\right)$ and $\mathfrak{C}_{h}\left(\gamma_{j}\right)$ are defined as follows:

$$
\begin{aligned}
& \mathfrak{C}_{c l}\left(\gamma_{j}\right)=\left\{f \in C^{\infty}\left(T^{*}\left(\mathbb{R}^{2} / \sigma\right)\right),\{f, \xi\}=\{f, y \eta\}=0 \text { near } \gamma_{j}\right\} ; \\
& \mathfrak{C}_{h}\left(\gamma_{j}\right)=\left\{P(h) \in \Psi^{0},\left[P, Q_{1}\right] \text { and }\left[P, Q_{2}\right] \text { are } \mathrm{O}\left(h^{\infty}\right) \text { near } \gamma_{j}\right\} .
\end{aligned}
$$

(Recall that in our coordinates, $\gamma_{j}=((\mathbb{R} \times\{0\}) / \sigma) \times\left\{c_{o}\right\} \times\{0\}$, where $c_{o}=H_{p}\left(\Lambda_{o}\right)$.) Because the symbols of $Q_{1}$ and $Q_{2}$ are polynomials of degree $\leqslant 2$, the operators in $\mathfrak{C}_{h}\left(\gamma_{j}\right)$ are exactly the Weyl quantisations of symbols of the form $\sum h^{k} a_{k}$ with $a_{k} \in \mathfrak{C}_{c l}\left(\gamma_{j}\right)$.

THEOREM 2.1 (Microlocal normal form). - There exists an elliptic Fourier integral operator $U(h)$ associated to the canonical transformation $\psi$ of Theorem 1.13, an invertible $2 \times 2$ matrix $\mathcal{M}(h)$ of pseudodifferential operators in $\mathfrak{C}_{h}\left(\gamma_{j}\right)$, and complex-valued functions of $h: \varepsilon_{1}(h)$ and $\varepsilon_{2}(h)$ admitting an asymptotic expansion in $\mathbb{C} \llbracket h \rrbracket$ :

$$
\varepsilon_{1}(h) \sim \sum_{\ell=0}^{\infty} \varepsilon_{1}^{(\ell)} h^{\ell}, \quad \varepsilon_{2}(h) \sim \sum_{\ell=0}^{\infty} \varepsilon_{2}^{(\ell)} h^{\ell}
$$

such that, microlocally near $\gamma_{j}$ :

$$
U^{-1}\left(\widehat{H}_{1}-a_{o}, \widehat{H}_{2}-b_{o}\right) U=\mathcal{M} \cdot\left(Q_{1}-\varepsilon_{1}, Q_{2}-h \varepsilon_{2}\right)+\mathrm{O}\left(h^{\infty}\right) .
$$

If $\widehat{H}_{1}$ and $\widehat{H}_{2}$ are formally self-adjoint, then $U(h)$ can be chosen to be microlocally unitary, and the functions $\varepsilon_{j}$ are real-valued.

- The first terms of $\varepsilon_{1}(h)$ (of order respectively $h^{0}$ and $h^{1}$ ) are given by the formulae:

$$
\begin{aligned}
\varepsilon_{1}^{(0)} & =c_{o}=\frac{1}{2 \pi} \int_{\delta} \alpha ; \\
\varepsilon_{1}^{(1)} & =\frac{1}{2 \pi} \int_{\delta} \kappa_{o}+\mu(\delta) / 4,
\end{aligned}
$$

where $\mu$ is the Maslov index of any regular part of $\Lambda_{o}$, and $\delta$ is any cycle associated to an $S^{1}$-orbit on $\Lambda_{o} \backslash \gamma$ (and recall that $\kappa_{o}$ is the sub-principal form of the system).

- The first term of $\varepsilon_{2}(h)$ is given by the formula:

$$
\varepsilon_{2}^{(0)}=\left(\frac{\lambda r_{1}-r_{2}}{\left|\mathcal{H}_{\Sigma}\left(H_{2}\right)\right|^{1 / 2}}\right)_{i \gamma_{j}},
$$

where $\lambda$ is defined in Lemma 1.7 (recall that $r_{i}$ is the sub-principal symbol of $\widehat{H}_{i}$ and $\mathcal{H}_{\Sigma}\left(H_{2}\right)$ is the transversal Hessian of $\left.H_{2}\right)$. Note that $\mathcal{H}_{\Sigma}\left(H_{2}\right)$ is also equal to the $(y, \eta)$-Hessian of $H_{2, j}$ (the latter was defined along with $\lambda$ in Lemma 1.7).

Remark 2.1. - Recall that there is a choice of sign in the canonical chart $\psi$ of Theorem 1.13. If the other sign is chosen, then $\varepsilon_{2}$ becomes $-\varepsilon_{2}$.

Proof. - Consider the direct case first. First take $U$ as any Fourier integral operator associated to $\psi$ (note that by construction this symplectomorphism is exact in the sense that it preserves the action integral). Since $H_{1}$ and $H_{2}$ commute with $H_{p}$ and $H_{q}$, Theorem 1.13 implies that the principal symbols of $U^{-1} \widehat{H}_{1} U$ and $U^{-1} \widehat{H}_{2} U$ are in the classical commutant $\mathfrak{C}_{c l}\left(\gamma_{j}\right)$. The following division lemma is easily proved as in [25]: 
Lemma 2.2. - Any function $K \in \mathfrak{C}_{c l}\left(\gamma_{j}\right)$ that vanishes on $\gamma_{j}$ can be written (in a neighbourhood of $\gamma_{j}$ ):

$$
K(x, \xi, y, \eta)=K(\xi, y, \eta)=a\left(\xi-c_{o}\right)+b y \eta
$$

for some smooth functions $a$ and $b$ in $\mathfrak{C}_{c l}\left(\gamma_{j}\right)$.

Applying this lemma to $H_{1} \circ \psi$ and $H_{2} \circ \psi$ solves the principal part of Eq. (9).

The next steps are obtained by conjugating $U$ by elliptic pseudodifferential operators, yielding transport equations of the form:

LEMMA 2.3. - Given any functions $\left(r_{1}, r_{2}\right)$ such that

$$
\left\{r_{1}, y \eta\right\}=\left\{r_{2}, \xi\right\}
$$

there exist $K_{1}, K_{2} \in \mathfrak{C}_{c l}\left(\gamma_{j}\right)$ and a function $f$ such that

$$
\{\xi, f\}=K_{1}-r_{1} \quad \text { and } \quad\{y \eta, f\}=K_{2}-r_{2} .
$$

Proof. - Let

$$
K_{1}(\xi, y, \eta)=\frac{1}{2 \pi} \int_{0}^{2 \pi} r_{1}(x, \xi, y, \eta) d x .
$$

Of course, $\left\{\xi, K_{1}\right\}=0$, and using the hypothesis of the lemma, one has

$$
\left\{K_{1}, y \eta\right\}=\frac{1}{2 \pi} \int_{0}^{2 \pi}\left\{r_{2}, \xi\right\} d x=\frac{1}{2 \pi} \int_{0}^{2 \pi}-\frac{\partial r_{2}}{\partial x} d x=0 .
$$

Now, let

$$
f_{1}(x, \xi, y, \eta)=\int_{0}^{x}\left(K_{1}(\xi, y, \eta)-r_{1}\left(x^{\prime}, \xi, y, \eta\right)\right) d x^{\prime} .
$$

Then $f_{1}$ is a smooth function on $T^{*}\left(\mathbb{R}^{2} / \sigma\right)$ that satisfies - using the same kind of calculation as above:

$$
\left\{f_{1}, y \eta\right\}(x, \xi, y, \eta)=r_{2}(x, \xi, y, \eta)-r_{2}(0, \xi, y, \eta) .
$$

Then the wanted function $f$ is sought under the form $f=f_{1}+f_{2}$, which leads to the system

$$
\left\{\xi, f_{2}\right\}=0 \quad \text { and } \quad\left\{y \eta, f_{2}\right\}=K_{2}-r_{2}(0, \xi, y, \eta) .
$$

It suffices to see $\xi$ as a parameter and apply a known lemma in the $(y, \eta)$-variables (see e.g. [18, Theorem 2]).

In the reverse case, the proof of the theorem is the same provided we deal with functions that are invariant under $T^{*} \sigma$. But if $K(\xi,-y,-\eta)=K(\xi, y, \eta)$, Lemma 2.2 still applies, yielding functions $a$ and $b$ with the same properties. The same is true for the transport equation. Then each step of the proof can be quantised via Weyl's formula to yield well-defined pseudodifferential operators on $\mathbb{R}^{2} / \sigma$. Thus the result still holds for the reverse case. 
The proofs for formulae (10), (11) and (12) are given in Section 2.4, but the formula for $\varepsilon_{1}$ is apparent in the proof of Theorem 2.4 below, and the formula for $\varepsilon_{2}$ can be directly checked using the fact that the subprincipal symbol is preserved under conjugation by an elliptic Fourier integral operator at a critical point of the principal symbol.

\subsection{Microlocal solutions}

We investigate here the solutions of the system

$$
\left(\widehat{H}_{1}(h)-a_{o}\right) u=\mathrm{O}\left(h^{\infty}\right), \quad\left(\widehat{H}_{2}(h)-b_{o}\right) u=\mathrm{O}\left(h^{\infty}\right),
$$

microlocally on a neighbourhood of the critical Lagrangian $\Lambda_{o}$. If the operators $\widehat{H}_{j}$ depend smoothly on some additional parameter $E \in \mathbb{R}^{d}$ that leaves the principal symbols intact, then all the results presented here depend smoothly and locally uniformly on $E$. This applies in particular to the investigation of the joint spectrum in a window of size $\mathrm{O}(h)$ around $\left(a_{o}, b_{o}\right)$, where $\widehat{H}_{j}$ is to be replaced by $\widehat{H}_{j}-h E_{j}$.

Theorem 2.1, applied to all critical circles $\gamma_{j}$, yields a finite set of semiclassical invariants $\left(\varepsilon_{1, j}(h), \varepsilon_{2, j}(h)\right)$. We show here how these quantities are related to the solutions of (13).

THEOREM 2.4 (The global quantum number).-

- The asymptotic series $\varepsilon_{1}(h)=\varepsilon_{1, j}(h)$, modulo $h \mathbb{Z}$, depend neither on $j$, nor on the particular way to achieve the normal form of Theorem 2.1.

- The system (13) admits a microlocal solution near any (and then all) $S^{1}$-orbit (including critical circles) if and only if the following condition holds:

$$
\varepsilon_{1}(h) \in h \mathbb{Z}+\mathrm{O}\left(h^{\infty}\right) .
$$

Remark 2.2. - Since $\varepsilon_{1}(h)$ is determined by $\widehat{H}_{1}(h)$ and $\widehat{H}_{2}(h)$, the fulfillment of Eq. (14) seems to impose a quantisation condition on $h$. While we can stick here to this interpretation, another possibility would be to recall that everything (and in particular $\varepsilon_{1}(h)$ ) smoothly depends on the point $o$ in the curve of critical values of $F$. Then Eq. (14) can be interpreted as a quantisation condition on $o$, which leaves $h$ free to vary in a full neighbourhood of 0 . This viewpoint is made clear in Section 2.4 (cf. Corollary 2.14).

Proof of Theorem 2.4. - We introduce the sheaf $\left(\mathfrak{L}, \Lambda_{o}\right)$ of germs of microlocal solutions on $\Lambda_{o}$, as a sheaf of $\mathbb{C}_{h}$-modules, where $\mathbb{C}_{h}$ is the ring of all complex functions of $h, c(h)$, such that

$$
|c(h)| \leqslant C \cdot h^{-N},
$$

for some constants $C, N$, modulo those functions that are $\mathrm{O}\left(h^{\infty}\right)$. Note that the vector operator $\widehat{F}=\left(\widehat{H}_{1}, \widehat{H}_{2}\right)$ acts on the huge sheaf over $\Lambda_{o}$ of germs of all admissible distributions modulo microlocal equivalence, and $\left(\mathfrak{L}, \Lambda_{o}\right)$ can be seen as the kernel of $\widehat{F}$. The question is to find out how local germs can be glued together to form a non-trivial global section of $\left(\mathfrak{L}, \Lambda_{o}\right)$, i.e. a solution of (13) near $\Lambda_{o}$.

It was shown in [26] that the restriction $\left(\mathfrak{L}, \Lambda_{o} \backslash \gamma\right)$ to the non-singular points of $F$ is a locally constant sheaf, and the germs $\mathfrak{L}(p)$ at any non-singular point $p$ form a free module of rank 1 , generated by a standard WKB solution. The existence of non-trivial global sections of $\left(\mathfrak{L}, \Lambda_{o} \backslash \gamma\right)$ is then characterised by the nullity $\left(\bmod \mathrm{O}\left(h^{\infty}\right)\right)$ of the associated holonomy (or "Bohr-Sommerfeld cocycle"):

$$
\lambda(h) \in \mathrm{H}^{1}\left(\Lambda_{o} \backslash \gamma, \mathbb{R} / 2 \pi \mathbb{Z}\right) .
$$


Since $\Lambda_{o} \backslash \gamma$ is a disjoint union of cylinders $\Lambda_{\{i, j\}}^{k}$, whose homology $H_{1}\left(\Lambda_{\{i, j\}}^{k}\right)$ is generated by the cycle represented by any oriented $S^{1}$-orbit, we get a finite set of holonomies $\lambda_{\{i, j\}}^{k}(h)$ characterising $\left(\mathfrak{L}, \Lambda_{o} \backslash \gamma\right)$.

Apply now Theorem 2.1. The system (13) is then, on a neighbourhood $\Omega$ of $\gamma_{j}$, equivalent to the following standard system:

$$
Q_{1} u=\varepsilon_{1, j} u, \quad Q_{2} u=h \varepsilon_{2, j} u .
$$

At any non-singular point $p \in \Lambda_{o} \cap \Omega \backslash \gamma_{j}$, the standard WKB $u$ solution generating $\mathfrak{L}(p)$ is therefore of the form

$$
u(p)=\mathrm{e}^{i \frac{\varepsilon_{1, j}}{h} x} v(y), \quad(x, y) \sim p \in \mathbb{R}^{2} / \sigma .
$$

This implies that

$$
\frac{1}{2 \pi} \int_{\delta} \lambda(h) \equiv \frac{\varepsilon_{1, j}(h)}{h}+\mathrm{O}\left(h^{\infty}\right) \quad(\bmod \mathbb{Z}),
$$

where $\delta$ is the cycle on $\Lambda_{o} \backslash \gamma$ associated with the orbit $S^{1}(p)$. This proves

1. that $\varepsilon_{1, j}(h)$ does not depend on the particular way to achieve the normal form;

2. that $\int_{\delta} \lambda(h)$ remains invariant if $p$ is chosen on another Lagrangian cylinder connecting $\gamma_{j}$ - which in turn proves

3. that $\varepsilon_{1}=\varepsilon_{1, j}$ does not depend on the choice of the critical circle $\gamma_{j}$ (since $\Lambda_{o}$ is connected). The first part of the theorem is now proved. Moreover, condition (14) is necessary and sufficient for the existence of a (non-trivial) solution near a regular orbit. This condition remains therefore necessary for the existence of a solution near a critical circle. We are thus left with the proof of the sufficiency of this condition for critical circles, which is achieved by the next proposition.

Proposition 2.5. - Let $\gamma_{j} \subset \gamma$ be a critical circle. Let $d=2$ or 4 be its degree in $G\left(\Lambda_{o}\right)$. If the condition (14) is fulfilled, then the set $\mathfrak{L}\left(\gamma_{j}\right)$ of germs of microlocal solutions on $\gamma_{j}$ is a free $\mathbb{C}_{h}$-module of rank $d / 2$.

Proof. - Let $n=n(h) \in \mathbb{Z}$ be such that $\varepsilon_{1}=h n+\mathrm{O}\left(h^{\infty}\right)$, and let $p \in \gamma_{j}$. We know from [8, Proposition 17] that the module of microlocal solutions of (15) at $p$ is free of rank 2 , generated by

$$
u_{ \pm} \stackrel{\text { def }}{=} \mathrm{e}^{i n x}\left(1_{ \pm y>0} \frac{1}{\sqrt{|y|}} \mathrm{e}^{i \varepsilon_{2} \ln |y|}\right) .
$$

If $\gamma_{j}$ is direct, this immediately implies that the module $\mathfrak{L}\left(\gamma_{j}\right)$ of microlocal solutions of (13) on the whole circle $\gamma_{j}$ is also free and of rank 2 .

In the reverse case, the distribution $C_{+} u_{+}+C_{-} u_{-}$on $\mathbb{T} \times \mathbb{R}$ is invariant under the involution $\sigma$ if and only if it has the parity of $n$ in the variable $y$, which reads here

$$
C_{-}=\mathrm{e}^{i n \pi} C_{+} .
$$

$\mathfrak{L}\left(\gamma_{j}\right)$ is in this case a free module of rank 1 , and its generator depends on the parity of $n$.

\subsection{The abstract Bohr-Sommerfeld rules}

We assume here that the first condition (14) is fulfilled, and show that the existence of global solutions of (15) can be read on the graph $G=G\left(\Lambda_{o}\right)$. As before, let $n=n(h) \in \mathbb{Z}$ be such that $\varepsilon_{1}=n+\mathrm{O}\left(h^{\infty}\right)$. 

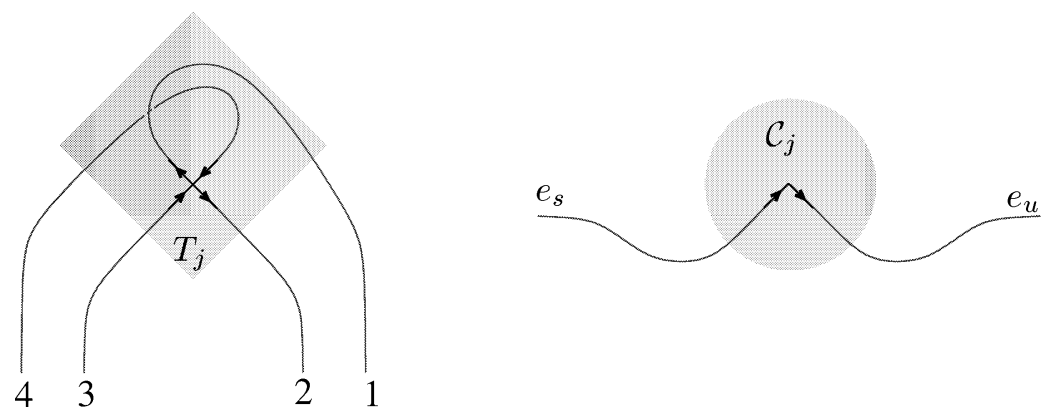

Fig. 4. Propagation of solutions at vertices of degree 4 and 2.

Because of Theorem 2.4, for any point $p \in \Lambda_{o}$, there exists a microlocal solution on a neighbourhood of the orbit $S^{1}(p)$. We shall use this fact to construct from the sheaf $\left(\mathfrak{L}, \Lambda_{o}\right)$ a new sheaf $(\overline{\mathfrak{L}}, G)$ on $G \subset W$ (recall that $W$ is the symplectic orbifold of Section 1.5) that will encode whether $\left(\mathfrak{L}, \Lambda_{o}\right)$ has a global section (see Theorem 2.7). Generalising the construction of [10], to each point $p \in G$ we associate the free module $\overline{\mathfrak{L}}(p)$ generated by the germs of standard basis at $p$, which will be of rank 1 , as follows.

Denote by $\bar{\gamma}_{j}$ the vertex of $G$ corresponding to the orbit $\gamma_{j}$, and let $\bar{\gamma}=\bigcup \bar{\gamma}_{j}$.

- At a regular orbit in $\Lambda_{o} \backslash \gamma$, a standard basis is just any basis of the space of solutions near $\gamma_{j}$, so we let $\overline{\mathfrak{L}}(p)=\mathfrak{L}\left(S^{1}(p)\right)$.

- At a vertex $\bar{\gamma}_{j}$ of degree 4 , a standard basis is defined in the following way.

The edges connecting $\bar{\gamma}_{j}$ are oriented according to the flow of $y \eta$. Moreover, near $\bar{\gamma}_{j}, W$ is a smooth oriented surface (the orientation is given by minus the symplectic form). It is shown in [8] that Proposition 2.5 in the direct case can be restated as follows: let $I_{1} I_{2}$ (resp. $I_{3} I_{4}$ ) be the disjoint union of the two local edges leaving $\bar{\gamma}_{j}$ (resp. arriving at $\bar{\gamma}_{j}$ ) with cyclic order $(1,3,2,4)$ - with respect to the orientation of $W$ near $\bar{\gamma}_{j} . \overline{\mathfrak{L}}\left(I_{1} I_{2}\right)$ and $\overline{\mathfrak{L}}\left(I_{3} I_{4}\right)$ are free modules of rank 2. Then there exists a linear map $T_{j}: \overline{\mathfrak{L}}\left(I_{3} I_{4}\right) \rightarrow \overline{\mathfrak{L}}\left(I_{1} I_{2}\right)$ such that $u$ is a solution in a neighbourhood of $\gamma_{j}$ if and only if its restrictions satisfy $u_{\overline{\mathfrak{L}}\left(I_{1} I_{2}\right)}=T_{j} u_{\overline{\mathfrak{L}}\left(I_{3} I_{4}\right)}$. In other words, if we "feed" the system with two functions on the entering edges $I_{3}$ and $I_{4}$, then these functions are propagated on the leaving edges $I_{1}$ and $I_{2}$ in a unique way (see Fig. 4). One can choose a basis element for each $\overline{\mathfrak{L}}\left(I_{i}\right), i=1, \ldots, 4$, and express $T_{j}$ as a $2 \times 2$ matrix $\left(\begin{array}{ll}a & b \\ c & d\end{array}\right)$ (defined modulo $\mathrm{O}\left(h^{\infty}\right)$ ). Moreover, one can show that the entries are all non-vanishing. It is then easy to check that a new choice for the basis elements does not change the cross-ratio $\rho_{j}=\frac{a d}{b c}$. In our situation, $\rho_{j}$ can be explicitly calculated: using a simple model yielding Eq. (18) below, one finds ([8]):

$$
\rho_{j}=\rho_{j}(h)=-\mathrm{e}^{2 \pi \varepsilon_{2, j}(h)} .
$$

The choice of a matrix $T_{j}$ fixes the basis elements up to their multiplication by a same factor. We shall call the choice of the basis elements of $\overline{\mathfrak{L}}\left(I_{i}\right), i=1, \ldots, 4$, a standard basis whenever $T_{j}$ has the following expression:

$$
T_{j}=\frac{1}{\sqrt{2 \pi h}} \Gamma(\beta) \mathrm{e}^{\beta \ln h}\left(\begin{array}{cc}
\mathrm{e}^{-i \beta \frac{\pi}{2}} & \mathrm{e}^{i \beta \frac{\pi}{2}} \\
\mathrm{e}^{i \beta \frac{\pi}{2}} & \mathrm{e}^{-i \beta \frac{\pi}{2}}
\end{array}\right),
$$

with $\beta=\frac{1}{2}+i \varepsilon_{2, j}$; or with the notations of [10]:

$$
T_{j}=T\left(\varepsilon_{2, j}(h)\right)=\mathrm{e}^{-i \frac{\pi}{4}} \mathcal{E}_{j}\left(\begin{array}{cc}
1 & i \mathrm{e}^{-\varepsilon_{2, j} \pi} \\
i \mathrm{e}^{-\varepsilon_{2, j} \pi} & 1
\end{array}\right),
$$


with

$$
\mathcal{E}_{j}=\mathcal{E}\left(\varepsilon_{2, j}(h)\right)=\frac{1}{\sqrt{2 \pi}} \Gamma\left(\frac{1}{2}+i \varepsilon_{2, j}\right) \mathrm{e}^{\varepsilon_{2, j}\left(\frac{\pi}{2}+i \ln h\right)} .
$$

Remark 2.3. - In [10] the factor $\mathrm{e}^{-i \frac{\pi}{4}}$ was absent in the definition of $T_{j}$ (19). Its introduction here will greatly simplify the treatment of Maslov indices (see also [5]).

Remark 2.4. - Eq. (17) proves that $\varepsilon_{2, j}$ is a semiclassical invariant (modulo $i \mathbb{Z}$ ) of the critical circle $\gamma_{j}$ : it does not depend on the particular way the normal form is achieved.

Remark 2.5. - As it is presented here, the notion of a standard basis seems to be attached to the graph $G$ endowed with a specific labelling at vertices of degree 4 . The form of the matrix $T_{j}$ shows that the different possible labellings of the four hyperbolic branches $I_{i}, i=1,2,3,4$, yield the same set of standard basis, provided $I_{1}$ and $I_{2}$ are the local unstable manifolds (for the flow of $y \eta$ - which means for the flow of $H_{q}$ ), $I_{3}$ and $I_{4}$ are the local stable manifolds, and on the oriented manifold $W$ (which is smooth at vertices of direct type) the branches appear in cyclic order $(1,3,2,4)$. Furthermore, it can be easily checked using the standard basis (25) given below and the fact that $\mathcal{F}_{h}^{2} f=\check{f}$ (where $\check{f}(y)=f(-y)$ ) that $T(\varepsilon) T(-\varepsilon)=\left(\begin{array}{ll}0 & 1 \\ 1 & 0\end{array}\right)$. Therefore, exchanging the local un/stable manifolds just amounts to changing the sign of $\varepsilon_{2, j}$.

- At a vertex $\bar{\gamma}_{j}$ of degree 2 , the space of solutions has dimension 1 , so we could just, as in the regular case, call any solution a standard basis. However, in order to isolate the "singular" components of the holonomy, we prefer the following convention which is more in accordance with the previous case (degree 4 ).

Let $I_{u}$ and $I_{s}$ be the local unstable and stable manifolds of $\bar{\gamma}_{j}$. A choice of basis elements $\left(e_{u}, e_{s}\right)$ for $\overline{\mathfrak{L}}\left(I_{u}\right)$ and $\overline{\mathfrak{L}}\left(I_{s}\right)$ will be called a standard basis if

$$
e_{u}=\mathcal{C}_{j} e_{s}
$$

with

$$
\begin{aligned}
\mathcal{C}_{j} & =\mathcal{C}\left(n, \varepsilon_{2, j}(h)\right)=\mathrm{e}^{-i \frac{\pi}{4}} \mathrm{e}^{-i n \frac{\pi}{2}} \mathcal{E}_{j}\left(1+i(-1)^{n} \mathrm{e}^{-\varepsilon_{2, j} \pi}\right) \\
& =\sqrt{\frac{2}{\pi h}} \Gamma(\beta) \mathrm{e}^{\beta \ln h} \cos \left(\frac{\pi}{2}(\beta+n)\right) .
\end{aligned}
$$

Notice that $\mathcal{C}_{j}$ depends on $n \bmod 4$.

Remark 2.6. - If $\varepsilon_{2, j} \in \mathbb{R}$, one has

$$
\mathcal{E}_{j}=\frac{1}{\sqrt{1+\mathrm{e}^{-2 \pi \varepsilon_{2, j}}}} \mathrm{e}^{i \arg \Gamma\left(1 / 2+i \varepsilon_{2, j}\right)+i \varepsilon_{2, j} \ln (h)} .
$$

Therefore $T_{j}$ is unitary and $\left|\mathcal{C}_{j}\right|=1$.

$(\overline{\mathfrak{L}}, G)$ is a locally flat sheaf of rank-one modules, and hence is characterised by its holonomy:

$$
\text { hol : } \mathrm{H}_{1}(G) \rightarrow \mathbb{C}_{h}
$$

In terms of Čech cohomology, if $\gamma$ is a loop in $G$, and $\Omega_{1}, \Omega_{2}, \ldots, \Omega_{\ell}=\Omega_{1}$ is an ordered sequence of open sets covering the image of $\gamma$, each $\Omega_{i}$ being equipped with a standard basis $u_{i}$, then

$$
\operatorname{hol}(\gamma) \stackrel{\text { def }}{=} x_{1,2} x_{2,3} \ldots x_{\ell-1, \ell}
$$






Fig. 5. Regularization of $\kappa_{o}$.

where $x_{i, j}$ is defined in $\mathbb{C}_{h}$ by $u_{i}=x_{i, j} u_{j}$ on $\Omega_{i} \cap \Omega_{j}$.

DEFINITION 2.2 (The singular invariants). -

1. The "principal value" $\tilde{\kappa}_{o}$ of the sub-principal form $\kappa_{o}$ is the cocycle on $\Lambda_{o}$ defined as follows:

- If $[A, B] \subset \Lambda_{o} \backslash \gamma$ is a non-singular path, then

$$
\int_{[A, B]} \tilde{\kappa}_{O}:=\int_{[A, B]} \kappa_{o}
$$

- If $[A, B] \subset \Lambda_{o}$ is a path intersecting once and transversally a unique critical circle $\gamma_{j}$, and oriented according to the flow of $H_{q}$ (i.e. $A$ is on the local stable manifold and $B$ is on the local unstable manifold) then

$$
\int_{[A, B]} \tilde{\kappa}_{o}:=\lim _{a, b \rightarrow m}\left(\int_{[A, a]} \kappa_{o}+\int_{[b, B]} \kappa_{o}+\varepsilon_{2, j}^{(0)} \ln \left|\int_{R_{a, b}} \omega\right|\right),
$$

where $R_{a, b}$ is the parallelogram (defined in any coordinate system) built on the vectors $\overrightarrow{m a}$ and $\overrightarrow{m b}\left(m=[A, B] \cap \gamma_{j}-\right.$ see Fig. 5).

2. The "regularized" Maslov index $\tilde{\mu}$ on $\Lambda_{o}$ is defined as follows:

- The contribution from a regular path in $\Lambda_{o} \backslash \gamma$ is the usual Maslov index of the path.

- Let $\delta=[A, B] \subset \Lambda_{o}$ be a small path intersecting once and transversally a unique critical circle $\gamma_{j}$, such that $A$ belongs to one of the hyperbolic branches $(1,3,2,4)$ and $B$ to an adjacent branch (i.e. $\delta$ makes a turn of angle $\pm \frac{\pi}{2}$ ). $\delta$ can be continuously deformed into a path $\delta_{t}$ drawn on a regular leaf of $F$. Then the usual Maslov index for this path is constant for $t$ small enough $\left(\delta_{0}=\delta\right)$, and we define

$$
\tilde{\mu}(\delta):=\mu\left(\delta_{t}\right) \pm\left(\frac{1}{2}+\chi_{\left\{d_{j}=2\right\}} n\right),
$$

where $\pm=$ " + " if $\delta$ turns in the direct sense (with respect to the cyclic order of the branches) and “_" otherwise, and $\chi_{\left\{d_{j}=2\right\}}=1$ if $\gamma_{j}$ is of degree 2 and $\chi_{\left\{d_{j}=2\right\}}=0$ otherwise. 

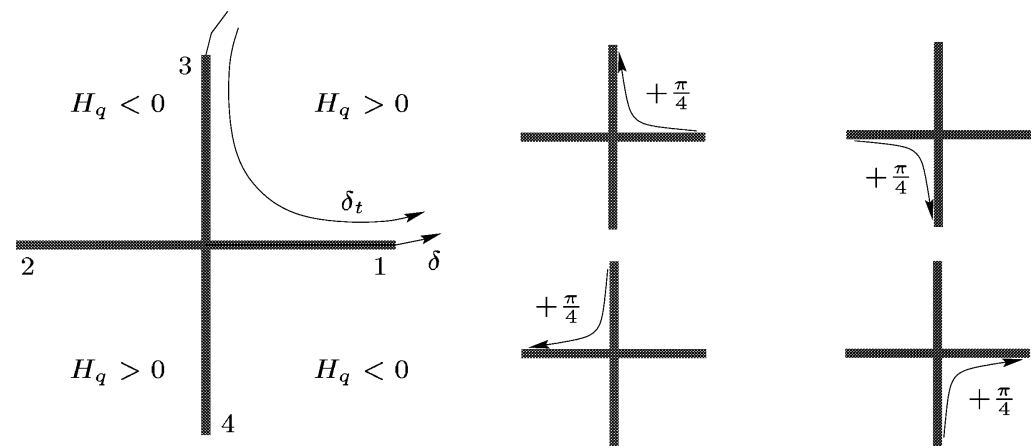

Fig. 6. The local Maslov correction (for a vertex of degree 2, replace $\frac{\pi}{4}$ by $\frac{\pi}{4}+n \frac{\pi}{2}$ ).

Remark 2.7. - The sign in (24) is negative if $\delta$ is oriented according to the flow of $H_{q}$ and $\delta_{t}$ belongs to a region in phase space where $H_{q}>0$, and changes whenever one of these two conditions changes. Of course the rule of Fig. 6 is simpler to use, but this correspondence will be used for the proofs of Section 2.4.

PROPOSITION 2.6. -

1. The holonomy of the sheaf $(\overline{\mathfrak{L}}, G)$ has the form hol $=\mathrm{e}^{i[\theta(h)] / h}$, where $\left[\pi^{*} \theta(h)\right] \in$ $\mathrm{H}^{1}\left(\Lambda_{o},(\mathbb{C},+)\right)$ admits an asymptotic expansion in non-negative powers of $h .\left(\pi: \Lambda_{o} \rightarrow G\right.$ is the projection associated to the $S^{1}$-reduction.)

2. Let $\sum_{\ell \geqslant 0}\left[\tilde{\theta}_{\ell}\right] h^{\ell}$ be the asymptotic expansion of $[\tilde{\theta}(h)]:=\pi^{*}[\theta(h)]$. Then the first two terms are given by the following formulae:

- $\left[\tilde{\theta}_{0}\right]=[\alpha]$ (the Liouville 1-form on $\left.\Lambda_{o}\right)$;

- $\left[\tilde{\theta}_{1}\right]=\left[\tilde{\kappa}_{o}\right]+\tilde{\mu} \frac{\pi}{2}$.

Proof. - We just prove here the existence of the claimed asymptotic expansion. The formulae for $\tilde{\theta}$ will follow from our refined analysis in Section 2.4 (Corollary 2.21).

For this purpose, it suffices to show that one can choose local sections $u_{\alpha}$ of $(\overline{\mathfrak{L}}, G)$ for which the transition constants $c_{\alpha, \beta}$ have the required form. On the edges of $G$, this follows from the regular theory of WKB solutions. At a critical circle, we apply the normal form (Theorem 2.1), and choose the following standard basis ( $u_{ \pm}^{\varepsilon_{2}}$ is defined in Eq. (16)):

- in the direct case,

$$
\left\{\begin{array}{l}
e_{1}=u_{+}^{\varepsilon_{2}}, \\
e_{2}=u_{-}^{\varepsilon_{2}} \\
e_{3}=\mathcal{F}_{h}^{-1}\left(u_{+}^{-\varepsilon_{2}}\right), \\
e_{4}=\mathcal{F}_{h}^{-1}\left(u_{-}^{-\varepsilon_{2}}\right)
\end{array}\right.
$$

- in the reverse case,

$$
\left\{\begin{array}{l}
e_{u}=e_{1}+(-1)^{n} e_{2}, \\
e_{s}=i^{n} e_{3}+(-i)^{n} e_{4} .
\end{array}\right.
$$

We see then that the restrictions of these solutions to any edge are standard WKB solutions, whose phases admit an asymptotic expansion in powers of $h$.

The dimension $b_{1}=\operatorname{dim} \mathrm{H}_{1}(G)$ is given by Euler-Poincaré formula:

$$
b_{1}=\#\{\text { edges of } G\}-N+1
$$


(recall that $N$ is the number of vertices of $G$ ). Moreover, if we write $N=p+q$ with $p$ the number of tetravalent vertices and $q$ the number of divalent vertices, then it is easy to see that

$$
\#\{\text { edges of } G\}=2 p+q,
$$

so that $b_{1}=p+1$.

We can now cut $b_{1}$ edges of $G$, each one corresponding to a cycle $\delta_{i}$ in a basis $\left(\delta_{1}, \ldots, \delta_{b_{1}}\right)$ of $\mathrm{H}_{1}(G)$, in such a way that the remaining graph is a tree $T\left(\mathrm{H}_{1}(T)=0\right)$. Then the sheaf $(\overline{\mathfrak{L}}, T)$ has a non-trivial global section, i.e. there exists a standard basis $u_{\alpha}$ on each edge $e_{\alpha}$ such that they extend to a standard basis at each vertex.

THEOREM 2.7.- $\left(\mathfrak{L}, \Lambda_{o}\right)$ has a non-trivial global section if and only if the following linear system of $3 p+q+1$ equations with the $3 p+q+1$ unknowns $\left(x_{\alpha} \in \mathbb{C}_{h}\right)_{\alpha \in\{\text { edges of } T\} \text { has a }}$ non-trivial solution:

1. if the edges $\left(\alpha_{1}, \alpha_{3}, \alpha_{2}, \alpha_{4}\right)$ connect at a tetravalent vertex $\gamma_{j}$ (with the prescribed orientation), then

$$
\left(x_{\alpha_{3}}, x_{\alpha_{4}}\right)=T_{j}\left(x_{\alpha_{1}}, x_{\alpha_{2}}\right) ;
$$

2. if the edges $\left(\alpha_{u}, \alpha_{s}\right)$ connect at a divalent vertex $\gamma_{j}$ (with the prescribed orientation), then

$$
x_{\alpha_{s}}=\mathcal{C}_{j} x_{\alpha_{u}}
$$

3. if $\alpha_{0}$ and $\alpha_{1}$ are the extremities of a cut cycle $\delta_{i}$, then

$$
x_{\alpha_{0}}=\operatorname{hol}\left(\delta_{i}\right) x_{\alpha_{1}} .
$$

Here we assume the following orientation: $\delta_{i}$ can be represented by a closed path starting on the edge $\alpha_{0}$ and ending on $\alpha_{1}$.

Remark 2.8. - When solving the system, it is immediate (if $p \neq 0$ ) to replace the equations of type (2) and (3) into those of type (1), in order to finally obtain a linear system of size $(2 p) \times(2 p)$. If $p=0$ then equations of type (2) and (3) combine together to yield a unique equation in one variable.

Proof. - Any global section $u$ of $\left(\mathfrak{L}, \Lambda_{o}\right)$ can be characterised by the set of constants $x_{\alpha} \in \mathbb{C}_{h}$ such that $u_{\uparrow \overline{\mathcal{L}}\left(e_{\alpha}\right)}=x_{\alpha} u_{\alpha}$. By definition of the standard basis $\left(u_{\alpha}\right)$, conditions (1) and (2) are necessary and sufficient for $\left(x_{\alpha} u_{\alpha}\right)$ to extend to a solution near every critical circle $\gamma_{j}$. It remains to check that the solutions at the extremities $\alpha_{0}, \alpha_{1}$ of a cut cycle $\delta_{i}$ can be consistently glued back together. Since $\left(u_{\alpha}\right)$ is a global section of $(\overline{\mathfrak{L}}, T), u_{\alpha_{1}}$ is the parallel transport of $u_{\alpha_{0}}$ along $\delta_{i}$, which means that, as local sections of $(\overline{\mathfrak{L}}, G)\left(\right.$ or $\left(\mathfrak{L}, \Lambda_{o}\right)$ ), they satisfy $u_{\alpha_{1}}=\operatorname{hol}\left(\delta_{i}\right) u_{\alpha_{0}}$. Therefore the solutions $x_{\alpha_{0}} u_{\alpha_{0}}$ and $x_{\alpha_{1}} u_{\alpha_{1}}$ can be glued back if and only if condition (3) holds.

\subsection{The spectral problem}

The goal of this section is to investigate uniform estimates for our system when it depends on spectral parameters. Specifically, we look now at the system

$$
\left(\widehat{H}_{1}(h)-E_{1}\right) u=\mathrm{O}\left(h^{\infty}\right), \quad\left(\widehat{H}_{2}(h)-E_{2}\right) u=\mathrm{O}\left(h^{\infty}\right),
$$

where $E_{1}$ and $E_{2}$ are real numbers. Here we shall assume that $\widehat{H}_{1}$ and $\widehat{H}_{2}$ are formally selfadjoint. If we are only interested in studying the spectrum in a window of size $\mathrm{O}(h)$ around 
the origin, we can let $E_{i}=h \varepsilon_{i}$ and there is nothing more to be done: Theorem 2.1 holds uniformly for $\left(\varepsilon_{1}, \varepsilon_{2}\right)$ varying in a compact set of $\mathbb{R}^{2}$, so that all the results of the previous sections apply. However, Theorem 2.1 does not apply to the system (26) with uniform estimates for $E=\left(E_{1}, E_{2}\right)$ in a compact. Indeed, it would imply that $H_{p}$ has a unique value on the local level set $\left(H_{1}, H_{2}\right)=\left(E_{1}, E_{2}\right)$ near $\gamma_{j}$; if $E$ is a regular value of $F$, this level set may fail to be connected and it is easy to construct a situation where $H_{p}$ has different values on each component. Actually, $H_{p}$ by definition is a function on the set of leaves of the Lagrangian foliation defined by $F$; and the following diagram is in general non-commutative:



Instead, we need to work with the space of leaves $\bar{\Omega}$, equipped with the momentum map $\bar{F}$.

For any $E \in U \subset\left(\mathbb{R}^{2}, 0\right)$, let $\Lambda_{E}=F^{-1}(E)$. If $U$ is a sufficiently small ball around some critical value, the curve $\mathcal{C}_{c} \subset U$ of critical values of the momentum map $F$ separates the set of regular values in $U$ into two simply connected open sets $U^{+}$and $U^{-}$. Using $H_{q}$ defined in Section 1.6, we take the following convention: $U^{ \pm}:=\left\{ \pm H_{q}>0\right\}$. Let $D^{ \pm}:=U^{ \pm} \cup \mathcal{C}_{c}$. Let $N^{+}$and $N^{-}$be the sets of connected components of the open sets $F^{-1}\left(U^{+}\right)$and $F^{-1}\left(U^{-}\right)$ respectively. In each of $U^{+}$and $U^{-}$, the level sets of $F$ have a unique topological type, namely they are unions of a finite number $\widetilde{N}^{ \pm}$of Liouville tori. The smooth family of tori in the component $k^{ \pm}$is denoted by $T_{k^{ \pm}}(E)$ : for any $E \in U^{ \pm}, F^{-1}(E)=: \bigsqcup_{k^{ \pm} \in N^{ \pm}} T_{k^{ \pm}}(E)$. Of course, $\widetilde{N}^{ \pm}=\left|N^{ \pm}\right|$.

PROPOSITION 2.8. - A smooth function $K$ commuting with $H_{1}$ and $H_{2}$ in $\Omega$ is characterised by the data of $\left|N^{+}\right|$functions $f_{k^{+}} \in C^{\infty}\left(D^{+}\right)$and $\left|N^{-}\right|$functions $f_{k^{-}} \in C^{\infty}\left(D^{-}\right)$such that

1. For all $k^{ \pm} \in N^{ \pm}, K_{\left\lceil k^{ \pm}\right.}=f_{k^{ \pm}} \circ F_{\left\lceil k^{ \pm}\right.}$;

2. For all $k^{+} \in N^{+}$and $k^{-} \in N^{-}$, the function equal to $f_{k^{+}}$in $D^{+}$and to $f_{k^{-}}$in $D^{-}$is smooth on $U$.

DEFINITION 2.3. - The space of smooth functions in $\Omega$ commuting with $H_{1}$ and $H_{2}$ will be denoted by $C^{\infty F}(\Omega)$. The space of leaves together with the smooth structure described in the above proposition will be called the Reeb graph of $F$.

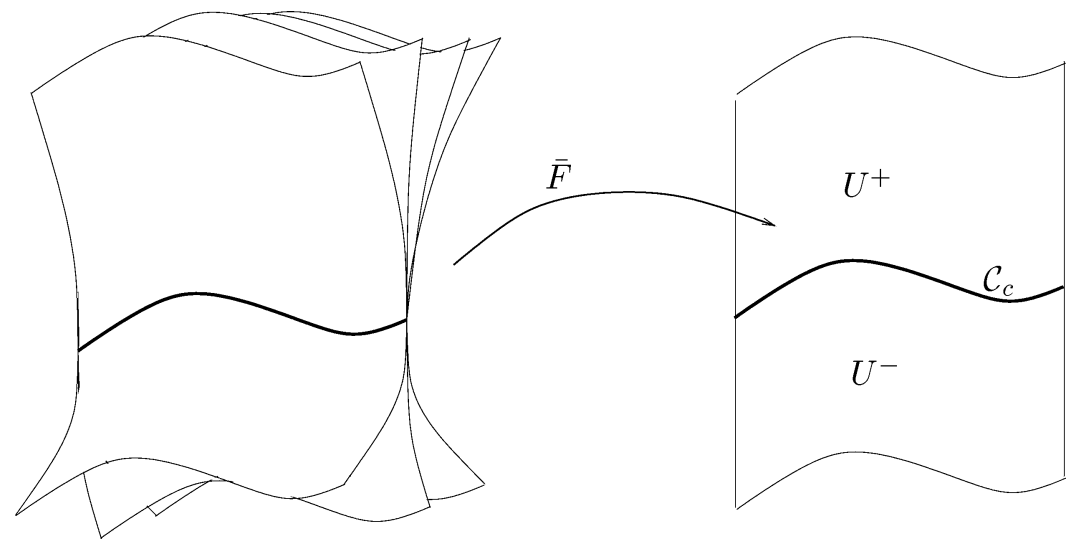

Fig. 7. The Reeb graph of $F$. 
Proof. - (a) Given $K$, the condition (1) uniquely defines the functions $f_{k^{ \pm}}$. Their smoothness is given by the Darboux-Carathéodory theorem that states that near every non-singular point of $\Lambda_{E}$, there is a canonical chart $(x, y, \xi, \eta)$ in which $H_{1}=\xi$ and $H_{2}=\eta$. The fact that such nonsingular points exist even on a singular leaf $\Lambda_{E_{c}}, E_{c} \in \mathcal{C}_{c}$, shows that the smoothness extends to $D^{ \pm}$. The same argument shows that condition (2) holds whenever $\bar{k}^{+} \cap \bar{k}^{-} \neq \emptyset$. Then condition (2) without this restriction holds because $\Lambda_{E_{c}}$ is connected.

(b) Conversely, the data of all the functions $f_{k^{ \pm}}$defines a unique function $K$. The smoothness of $K$ outside of the critical points comes from the same argument as above; its smoothness at critical points comes from Theorem 1.5 and the following Lemma 2.9.

LEMMA 2.9. - Each function $K$ commuting with $\xi$ and y $\eta$ near $(x, 0,0,0)$ is characterised by two functions $f_{+}, f_{-} \in C^{\infty}\left(\mathbb{R}^{2}, 0\right)$ satisfying

$$
f_{-}(\xi, t)-f_{+}(\xi, t)=\mathrm{O}\left(t^{\infty}\right)
$$

locally uniformly in $\xi$, such that

$$
K(x, y, \xi, \eta)= \begin{cases}f_{+}(\xi, y \eta) & \text { if } y \geqslant 0 \\ f_{-}(\xi, y \eta) & \text { if } y<0\end{cases}
$$

Proof. - The existence of the functions $f_{+}$and $f_{-}$is equivalent to the fact that $K$ is independent of $x$ and locally constant on the fibers $y \eta=$ const. Eq. (28) is then equivalent to the smoothness of $K$ around the axis $y=0$ and $\eta=0$.

Remark 2.9. - The distinction between the functions $f_{+}$and $f_{-}$is of course irrelevant when all the data is analytic. Neither has it any impact for semi-excited regions, i.e. for $E=\mathrm{O}\left(h^{\gamma}\right)$, $0<\gamma<1$ (see [26, Chapter 5]). In these cases, no further modification of the results of the previous sections is required (diagram (27) is always commutative), and the following Section 2.4.1 becomes rather straightforward. We have laid down all the details to cope with the $C^{\infty}$ case, which makes the statements and proofs more technical. On the other hand, the statements in Section 2.4.2 are non-trivial even in the analytic case, and represent some of the most crucial results of this article.

\subsubsection{The "global" quantum number}

In this section, the issue is to generalise the "global" quantum number $\varepsilon_{1}(h)$ of Theorem 2.4. In the smooth, non-analytic category, this leads to a subtle repartition property for the semiclassical spectrum.

For any $E \in U \backslash \mathcal{C}_{c}$, denote by $\left(\mathfrak{L}, \Lambda_{E}\right)$ the sheaf of germs of microlocal solutions of (26) on $\Lambda_{E}$. We know from the regular theory of Bohr-Sommerfeld rules on Liouville tori that $\left(\mathfrak{L}, \Lambda_{E}\right)$ is just a flat bundle of rank 1 characterised by its holonomy $\lambda_{h} \in \mathrm{H}^{1}\left(\Lambda_{E}, \mathbb{R} / 2 \pi \mathbb{Z}\right)$. When $E$ is restricted to any compact subset $K \subset U^{ \pm}$, and $\lambda_{h}$ is restricted to some connected component $k^{ \pm}$, $h \lambda_{h}$ has a uniform asymptotic expansion in $C^{\infty}(K) \llbracket h \rrbracket$. This is no longer true on $D^{ \pm}$. However, the following statement holds:

THEOREM 2.10. - The function that assigns to a leaf $T_{k^{ \pm}}(E)$ the integral

$$
\frac{h}{2 \pi} \int_{\delta} \lambda_{h}
$$


where $\delta$ is any $S^{1}$-orbit in $T_{k^{ \pm}}(E)$, defines an element $\mathfrak{e}_{1}(h) \in C^{\infty F}(\Omega)$ that admits an asymptotic expansion of the form:

$$
\mathfrak{e}_{1}(h)=\sum_{\ell=0}^{\infty} h^{\ell} \mathfrak{e}_{1}^{(\ell)} \in C^{\infty F}(\Omega) \llbracket h \rrbracket,
$$

with $\mathfrak{e}_{1}^{(0)}=H_{p}$, and $\mathfrak{e}_{1}^{(1)}=-\frac{a}{2 \pi} \int_{\delta} r_{1}-\frac{b}{2 \pi} \int_{\delta} r_{2}+\mu(\delta) / 4$, where $r_{j}$ is the sub-principal symbol of $\widehat{H}_{j}$, and $a, b$ in $C^{\infty} F(\Omega)$ are the functions defined by $\mathcal{X}_{p}=a \mathcal{X}_{1}+b \mathcal{X}_{2}$, and $\mu$ is the Maslov cocycle of $T_{k^{ \pm}}(E)$.

Remark 2.10. - Fix $k^{ \pm} \in N^{ \pm}$and realise $\left(\mathfrak{e}_{1}\right)_{\uparrow_{k^{ \pm}}}$as a smooth function $E \rightarrow \mathfrak{e}_{1}(E ; h)$ in $C^{\infty}\left(D^{ \pm}\right)$. Now let $E=\left(a_{o}, b_{o}\right)+h\left(e_{1}, e_{2}\right)$, where $e_{j}$ varies in a compact (recall that $\left(a_{o}, b_{o}\right)$ is a critical value of $F)$; then $\mathfrak{e}_{1}(E ; h)$ admits an asymptotic expansion in powers of $h$ whose coefficients are smooth functions of $\left(e_{1}, e_{2}\right)$. But these coefficients are made out of the Taylor series of $E \rightarrow \mathfrak{e}_{1}(E ; h)$, and therefore, in view of the definition of $C^{\infty F}$, they do not depend on the component $k^{ \pm}$. We obtain this way an element $\varepsilon_{1}(h) \in C^{\infty} \llbracket h \rrbracket$ that is nothing else but the global quantum number of Theorem 2.4.

Notice that, in order to compute the principal term in $\varepsilon_{1}(h)$, it is not interesting to use the formula $\varepsilon_{1}(h)=\mathfrak{e}_{1}(E ; h)$, since it would involve the derivative of $\mathfrak{e}_{1}^{(0)}$. Instead, apply the formula of the theorem above to a system whose principal symbol is independent of $E$, and whose subprincipal symbol is $\left(r_{1}-e_{1}, r_{2}-e_{2}\right)$. We obtain this way the claim (10)-(11) in Theorem 2.1.

Proof of the theorem. - The fact that $\mathfrak{e}_{1}(h) \in C^{\infty} F(\Omega)$ is obvious form the construction. To prove the existence of the claimed asymptotic expansion, it suffices to microlocalize near a critical circle $\gamma_{j}$.

Using Lemma 2.9 in the coordinates of Theorem 1.13, one sees that the functions $f_{+}$and $f_{-}$ of Lemma 2.9 are the same if the degree $d=2$. In this case, Theorem 2.1 generalises to

$$
U^{-1}\left(\widehat{H}_{1}-E_{1}, \widehat{H}_{2}-E_{2}\right) U=\mathcal{M} \cdot\left(Q_{1}-\mathfrak{e}_{1}, Q_{2}-\mathfrak{e}_{2}\right)+\mathrm{O}\left(h^{\infty}\right),
$$

where $\mathcal{M}, \mathfrak{e}_{1}$, and $\mathfrak{e}_{2}$ depend smoothly on $E$, which gives the result.

The case $d=4$ is more intricate, and follows from Proposition 2.12 below.

We shall need the following slightly weaker version of Theorem 2.1.

PROPOSITION 2.11. - There exists an elliptic Fourier integral operator $U(h)$ associated to the canonical transformation $\psi$ of Theorem 1.13 such that, microlocally near $\gamma_{j}$ :

$$
U^{-1} \widehat{H}_{j} U=\widehat{K}_{j}
$$

where $\widehat{K}_{j} \in \mathfrak{C}_{h}\left(\gamma_{j}\right)$.

Proof. - The same proof scheme as that of Theorem 2.1 applies, using Lemma 2.9 instead of Lemma 2.2.

Proposition 2.12. - Let $\gamma_{j}$ be a critical circle of degree 4. For each E close to zero, the set of microlocal solutions of (26) on a small neighbourhood of any point of $\gamma_{j}$ is a free $\mathbb{C}_{h}$-module of rank 2. In the coordinates of Theorem 1.13, it has a basis of the form

$$
u_{E}^{ \pm}=\mathrm{e}^{i \mathfrak{e}_{1}^{ \pm} x / h}\left(1_{ \pm y>0} \frac{1}{\sqrt{|y|}} \mathrm{e}^{i \mathfrak{e}_{2}^{ \pm} \ln |y| / h}\right)
$$


where $\mathfrak{e}_{j}^{ \pm}=\mathfrak{e}_{j}^{ \pm}(E ; h)$ admits an asymptotic expansion in non-negative powers of $h$ whose coefficients are smooth functions of $E$. For this basis, the system (26) is solved locally uniformly with respect to $\left(E_{1}, E_{2}\right)$ near $(0,0)$. Moreover, the functions $\left(E_{1}, E_{2}\right) \rightarrow \mathfrak{e}_{j}^{+}-\mathfrak{e}_{j}^{-}$are flat on the set $\mathcal{C}_{c}$ of critical points of $F$.

The proof of this proposition relies on the following lemma:

LEMMA 2.13. - Let $p \in C_{0}^{\infty}\left(T^{*} S^{1} \times T^{*} \mathbb{R}\right)$ be a Hamiltonian satisfying

$$
p(x, \xi, y, \eta)=0 \quad \text { for } y \geqslant 0
$$

(i.e. $p=1_{y \leqslant 0}$ ). Then

$$
O p_{h}^{W}(p)=O p_{h}^{W}(p) \circ 1_{y \leqslant 0}=1_{y \leqslant 0} . O p_{h}^{W}(p) \quad\left(\bmod \mathrm{O}\left(h^{\infty}\right)\right) .
$$

Of course the symmetric result (with respect to $y=0$ ) holds.

Proof. - Recall that Weyl quantisation of $p$ is defined by:

$$
\begin{aligned}
v(x, y) & =O p_{h}^{W}(p) u(x, y) \\
& =\frac{1}{(2 \pi h)^{2}} \int \mathrm{e}^{\frac{i}{h}\left(\left(x-x^{\prime}\right) \xi+\left(y-y^{\prime}\right) \eta\right)} p\left(\frac{x+x^{\prime}}{2}, \xi, \frac{y+y^{\prime}}{2}, \eta\right) u\left(x^{\prime}, y^{\prime}\right) d x^{\prime} d y^{\prime} d \xi d \eta .
\end{aligned}
$$

We prove the first estimate by showing that $\|v\|=\mathrm{O}\left(h^{\infty}\right)$ whenever $u=1_{y \geqslant 0} u$. For this, we consider the two regions $|y| \geqslant h^{\delta_{1}}$ and $|y| \leqslant h^{\delta_{2}}$, with $0<\delta_{2} \leqslant \delta_{1}<1$. If $|y| \leqslant h^{\delta_{2}}$, then only the domain $\left|\frac{y+y^{\prime}}{2}\right| \leqslant h^{\delta_{2}}$ contributes to the integral; and the result follows from the flatness of $p$ with respect to its third variable: for all $N \in \mathbb{N},|v|=\mathrm{O}\left(h^{N \delta_{2}}\right)$. Let us now look at the region $|y| \geqslant h^{\delta_{1}}$. Since $v(\cdot, \cdot, y \geqslant 0, \cdot)=0$, one can assume that $y \leqslant-h^{\delta_{1}}$, which implies $\left|y-y^{\prime}\right| \geqslant h^{\delta_{1}}$. Now the usual trick applies: a repeated integration by parts with respect to the operator $\frac{h}{i\left(y-y^{\prime}\right)} \frac{\partial}{\partial \eta}$ (or standard estimates for the Fourier transform) gives $|v|=\mathrm{O}\left(h^{N\left(1-\delta_{1}\right)}\right)$ for any integer $N$.

The same method can be applied to show that $\left\|1_{y \geqslant 0} v\right\|=\mathrm{O}\left(h^{\infty}\right)$ whenever $u=1_{y \leqslant 0} u$, thus proving the second estimate.

Proof of Proposition 2.12. - The fact that the set of solutions is a free module of rank 2 is due, for $E \notin \mathcal{C}_{c}$, to the regular theory (the local Lagrangian manifold has two connected components, on each of which the set of solutions is a free module of rank 1), and, for $E \in \mathcal{C}_{c}$, to Proposition 2.5.

We prove the rest of the proposition for $u_{E}^{+}$; the same argument applies to $u_{E}^{-}$. First apply Proposition 2.11 to assume in what follows that $\widehat{H}_{j} \in \mathfrak{C}_{h}\left(\gamma_{j}\right)$. Since $y \eta$ is a quadratic function, every element of $\widehat{K} \in \mathfrak{C}_{h}\left(\gamma_{j}\right)$ can be written $\widehat{K}=O p_{h}^{W}\left(K_{h}\right)$, with $K_{h} \sim \sum_{\ell \geqslant 0} h^{\ell} K^{(\ell)}$ and $K^{(\ell)} \in \mathfrak{C}_{c l}\left(\gamma_{j}\right)$. Because of Lemma 2.9, each $K^{(\ell)}$ is defined by two functions $f_{ \pm}^{(\ell)}$. Let

$$
F_{h}(x, \xi, y, \eta) \sim \sum_{\ell \geqslant 0} h^{\ell} f_{+}^{(\ell)}(\xi, y \eta)
$$

and $R_{h}=K_{h}-F_{h}$. Let us prove now that there is a unique symbol $g_{h}(\xi, t) \sim \sum_{\ell \geqslant 0} h^{\ell} g^{(\ell)}(\xi, t)$ such that

$$
O p_{h}^{W}\left(F_{h}\right)=g_{h}\left(Q_{1}, Q_{2}\right)+\mathrm{O}\left(h^{\infty}\right)
$$


$\left(Q_{j}\right.$ is defined in Eq. (8)). Indeed, $g^{(0)}$ is necessarily equal to $f_{+}^{(0)}$; therefore,

$$
O p_{h}^{W}\left(F_{h}\right)=g^{(0)}\left(Q_{1}, Q_{2}\right)+h \widehat{S},
$$

where $\widehat{S} \in \mathfrak{C}_{h}\left(\gamma_{j}\right)$ and is of order 0 . Then $\widehat{S}$ is similarly decomposed - and the claim is proved by induction - provided we show that its Weyl symbol is, as for $F_{h}$, a function of $(\xi, y \eta)$. This is achieved by applying Lemma 2.9 and remarking that $O p_{h}^{W}\left(F_{h}\right)$, as well as $g^{(0)}\left(Q_{1}, Q_{2}\right)$, commute with the involution $y \rightarrow-y$ (and thus their Weyl symbols are invariant under $(y, \eta) \rightarrow(-y,-\eta))$. Summing up, we have proved so far that any operator $\widehat{K} \in \mathfrak{C}_{h}\left(\gamma_{j}\right)$ can be written:

$$
\widehat{K}=g_{h}\left(Q_{1}, Q_{2}\right)+O p_{h}^{W}\left(R_{h}\right),
$$

where all the coefficients in the expansion of $R_{h}$ are smooth functions verifying the hypothesis of Lemma 2.13 .

Applying this to $\widehat{H}_{j}$, we obtain the existence of two symbols $g_{1, h}$ and $g_{2, h}$ such that

$$
\widehat{H}_{j} u_{E}^{+}=g_{j, h}\left(\mathfrak{e}_{1}^{+}, \mathfrak{e}_{2}^{+}\right) u_{E}^{+}+\mathrm{O}\left(h^{\infty}\right) .
$$

The independence of $H_{1}$ and $H_{2}$ implies that the principal term:

$$
(\xi, t) \rightarrow\left(g_{1}^{(0)}(\xi, t), g_{2}^{(0)}(\xi, t)\right)
$$

is local diffeomorphism; therefore the symbol $\left(g_{1, h}, g_{2, h}\right)$ is invertible, and the proposition is proved with

$$
\left(\mathfrak{e}_{1}^{+}, \mathfrak{e}_{2}^{+}\right) \sim\left(g_{1, h}, g_{2, h}\right)^{-1}\left(E_{1}, E_{2}\right)
$$

Corollary 2.14. - Fix $k^{ \pm} \in N^{ \pm}$and realise $\left(\mathfrak{e}_{1}\right)_{\left\lceil k^{ \pm}\right.}$as a smooth function $E \rightarrow \mathfrak{e}_{1}(E ; h)$ in $C^{\infty}\left(D^{ \pm}\right)$. Then the condition

$$
\mathfrak{e}_{1}(E ; h) \in h \mathbb{Z}+\mathrm{O}\left(h^{\infty}\right)
$$

is necessary and sufficient for the existence of a uniform solution of (26) microlocalised in a neighbourhood (in $\bar{k}^{ \pm}$) of any $S^{1}$-orbit in $\bar{k}^{ \pm}$.

DEFINITION 2.4. - For any natural integers $\ell^{+}$and $\ell^{-}$, we call an $\left(\ell^{+}, \ell^{-}\right)$-curve the union of $\ell^{+}$smooth curves in $D^{+}$and $\ell^{-}$smooth curves in $D^{-}$that are transversal to $\mathcal{C}_{c}$ and infinitely tangent to each other on $\mathcal{C}_{c}$.

These curves are just the image by $F$ of a level set of a smooth function $K$ on the Reeb graph of $F$, if $\mathcal{X}_{K} \neq 0$ on the critical leaves $\Lambda_{E_{c}}$ (this is a consequence of Proposition 2.8). This holds for instance for $K=H_{p}$ (Fig. 8).

COROLlARY 2.15. - There exists a fixed neighbourhood $U$ in $\mathbb{R}^{2}=\left\{\left(E_{1}, E_{2}\right)\right\}$ of any critical value of $F$ in which the joint spectrum of $\mathfrak{H}_{1}$ and $\widehat{H}_{2}$ is distributed (modulo $\mathrm{O}\left(h^{\infty}\right)$ ) on the union of $\left(\left|N^{+}\right|,\left|N^{-}\right|\right)$-curves $L_{n}(h)(n \in \mathbb{Z})$ defined as the image by $F$ of the level sets $\mathfrak{e}_{1}(h)=h n$. The principal part of these curves is thus given by the level sets of $H_{p}$.

Proof. - Fix $k^{ \pm} \in N^{ \pm}$. Then Proposition 2.8 says that the restriction $\left(\mathfrak{e}_{1}(h)\right)_{\lceil\bar{k} \pm}$ is equal to $f_{h} \circ F_{\left\lceil\bar{k}^{ \pm}\right.}$for some smooth function $f_{h} \sim \sum_{\ell=0}^{\infty} f_{\ell} h^{\ell}$ admitting an asymptotic expansion in 




Fig. 8. Level sets of $H_{p}$.

$C^{\infty}\left(\mathbb{R}^{2}, 0\right) \llbracket h \rrbracket$. Since $f_{0} \circ F_{\left\lceil\bar{k}^{ \pm}\right.}=\left(H_{p}\right)_{\left\lceil\bar{k}^{ \pm}\right.}$, the hypothesis (6) of Theorem 1.6 implies that the foliation $f_{h}=$ const is transversal to $\mathcal{C}_{c}$, and we can define the projection $\varpi_{h}: \mathbb{R}^{2} \rightarrow \mathcal{C}_{c}$ such that $\varpi_{h}(f=$ const $)$ is a point. The pre-image of $\left\{f_{h}=\right.$ const $\} \cap D^{ \pm}$by $F_{\left\lceil\bar{k}^{ \pm}\right.}$is a leaf of the foliation $\left\{\mathfrak{e}_{1}(h)=\right.$ const $\}$ in $\bar{k}^{ \pm}$. The value of $H_{p}$ on $\mathcal{C}_{c}$ can be taken as a coordinate on $\mathcal{C}_{c}$, and via this identification, it is natural to view $\mathfrak{e}_{1}(h)$ as a function with values in $\mathcal{C}_{c}$. By Corollary 2.14, any microlocal eigenfunction of $\left(\widehat{H}_{1}, \widehat{H}_{2}\right)$ microlocalised in $\bar{k}^{ \pm}$defines a joint eigenvalue that belongs to $\varpi_{h}^{-1}\left(h \mathbb{Z}+\mathrm{O}\left(h^{\infty}\right)\right)$

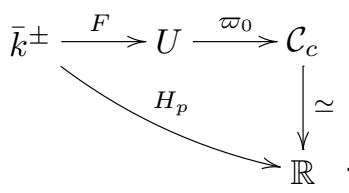

\subsubsection{Regularization of $\lambda_{h}(E)$}

This section contains some of the most central results of this article (Theorems 2.19 and 2.20). They give a new interpretation of the holonomy of Section 2.3 and provide for the proofs of the various formulae claimed before.

To each $k^{ \pm} \in N^{ \pm}$and $E_{c} \in \mathcal{C}_{c}$ we associate the subset $T_{k^{ \pm}}\left(E_{c}\right)=\bar{k}^{ \pm} \cap \Lambda_{E_{c}}$ which is the "limit" of the torus $T_{k^{ \pm}}(E)$ as $E \rightarrow E_{c}$. Suppose we are given a continuous family of piecewise differentiable loops $\left(\delta_{E}\right)_{E \in D^{ \pm}}$on $T_{k^{ \pm}}(E)$ that are everywhere transversal to the $S^{1}$-orbits (such a family can be constructed using for instance the normal form of Theorem 1.13), and assume that they are oriented by the flow of $H_{q}$. For non-singular values of $E, \delta_{E}$ together with an $S^{1}$ orbit form a basis of $\mathrm{H}_{1}\left(T_{k^{ \pm}}(E)\right)$, and $E \rightarrow \int_{\delta_{E}} \lambda_{h}$ is a smooth function. To complete the result of Theorem 2.10, it is natural to investigate here the behaviour of that function as $E$ approaches a critical value.

To each $E_{c} \in \mathcal{C}_{c}$ corresponds a real number $x$ via the diffeomorphism (30); $T_{k^{ \pm}}\left(E_{c}\right)$ is an $S^{1}$-invariant subset of $H_{p}^{-1}(x)$, and hence can be reduced to a cycle $G_{k^{ \pm}}\left(E_{c}\right)$ of the graph $G\left(E_{c}\right):=G\left(\Lambda_{E_{c}}\right)$ in the reduced manifold $W\left(E_{c}\right)$. The second goal of this section is to show how the asymptotic behaviour of the function $E \rightarrow \int_{\delta_{E}} \lambda_{h}$ is related to the holonomy hol of Section 2.3. Actually we shall prove that $\int_{\delta_{E}} \lambda_{h}$ diverges as $E$ approaches a critical value; but there is a universal way of regularizing this divergence. The regularized value is precisely 


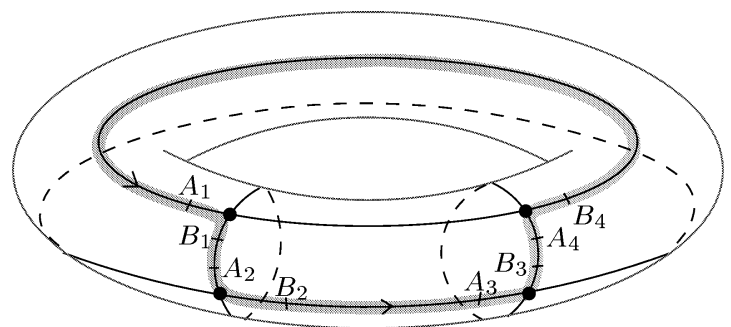

Fig. 9. A graph on a torus. The cycle in gray cannot be obtained as a combination of boundary faces of the graph.

$\operatorname{hol}\left(\bar{\delta}_{E}\right)$ - modulo some Maslov corrections in presence of vertices of degree 2 - where $\bar{\delta}_{E}$ is the projection of $\delta_{E}$ onto the reduced manifold and is actually equal to $G_{k^{ \pm}}\left(E_{c}\right)$.

Unfortunately, the set of cycles $G_{k^{ \pm}}\left(E_{c}\right)$ does not necessarily generate the group $\mathrm{H}_{1}\left(G\left(E_{c}\right), \mathbb{Z}\right)$ - see e.g. Fig. 9 - (but it does indeed if $G\left(E_{c}\right)$ is planar). So there is a little bit more to it than just taking the limit of regular cycles.

To compute hol of all possible cycles of $G\left(E_{c}\right)$, we have to replace the "natural" object $\delta_{E}$ with a local path near a critical circle. In order to give some sense to the expression $\int_{\delta_{E}} \lambda_{h}$, where $\delta_{E}$ is not closed, we could abstractly choose a smooth family of closed 1-forms on $\Lambda_{E}$, $E \in U^{ \pm}$, whose cohomology class is $\left[\lambda_{h}(E)\right]$ - which is always possible as $\mathrm{H}^{1}\left(\Lambda_{E}\right) \rightarrow U^{ \pm}$is a trivial bundle if $U$ is small enough. However, this does not allow us to have a local control of the divergence of the holonomy. Instead, we interpret $\lambda_{h}$ as the phase of the multiplicative Čech holonomy of the sheaf $\left(\mathfrak{L}, \Lambda_{E}\right)$, as follows:

Definition 2.5. - Near each $\Gamma_{j}$, we let $A_{j}(E)$ and $B_{j}(E)$ in $C^{\infty}\left(D^{ \pm}, \bar{k}^{ \pm} \backslash \Gamma_{j}\right)$ be families of points such that for a critical value $E_{c}, A\left(E_{c}\right)$ and $B\left(E_{c}\right)$ lie respectively in the local stable or unstable manifold. We endow a small neighbourhood of $A_{j}$ (or $B_{j}$ ) with a standard WKB microlocal solution $u_{A_{j}}$ (resp. $u_{B_{j}}$ ) whose phase admits an asymptotic expansion in $h^{-1} C^{\infty}\left(D^{ \pm}\right) \llbracket h \rrbracket$.

Then the integrals $\int_{A_{j}}^{B_{j^{\prime}}} \lambda_{h}$ are defined as the phase of the Čech holonomy of $\left(\mathfrak{L}, \Lambda_{E}\right)$ for paths joining $A_{j}$ and $B_{j^{\prime}}$ with the sections $u_{A_{j}}$ and $u_{B_{j^{\prime}}}$ fixed.

In other words, if the path $\delta$ between $A_{j}$ and $B_{j^{\prime}}$ is covered by open sets $\Omega_{0}, \ldots, \Omega_{\ell}$, each of which being endowed with a microlocal solution $u_{\alpha}$ with $u_{0}=u_{A_{j}}$ and $u_{\ell}=u_{B_{j^{\prime}}}$, then

$$
\int_{\delta} \lambda_{h}=-i \ln \left(c_{0,1} c_{1,2} \ldots c_{\ell-1, \ell}\right)
$$

where $c_{i, j}$ is the transition constant $u_{i}=c_{i, j} u_{j}$ on $\Omega_{i} \cap \Omega_{j}$.

Note that if another admissible choice for the local sections $u_{A / B}$ is made, then the holonomy is modified by an additive term admitting an asymptotic expansion in $h^{-1} C^{\infty}\left(D^{ \pm}\right) \llbracket h \rrbracket$. Therefore the singular behaviour of the holonomy at a critical value is fully preserved. Note also that this additive term is necessarily a Čech coboundary, and hence has no influence on the value of the holonomy along a closed loop.

DEFINITION 2.6. - In what follows, $\left(\delta_{E}\right)_{E \in D^{ \pm}}$designates a continuous family of paths in $T_{k^{ \pm}}(E)$ such that:

- for each $E \in U^{ \pm}, \delta_{E}$ is smooth; 


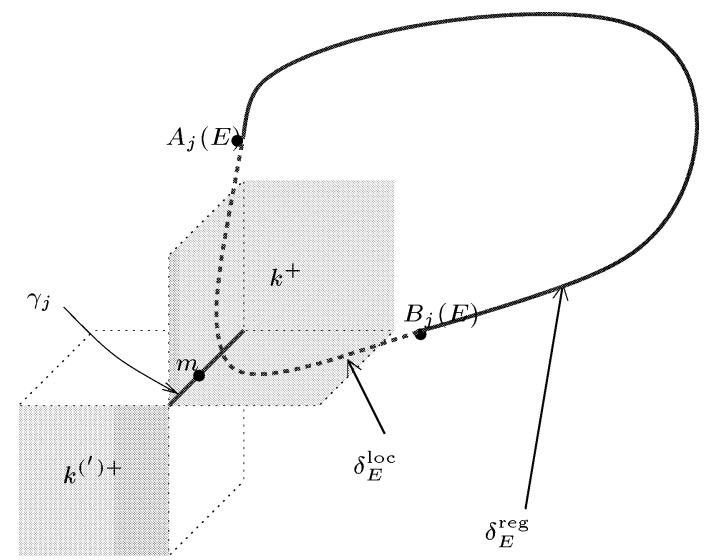

Fig. 10. "Regular" and "local" paths.

- either for all $E_{c} \in \mathcal{C}_{c}, \delta_{E_{c}}$ does not meet the critical set $\Gamma$ (then $\left(\delta_{E}\right)$ is called regular) or for each $E_{c}, \delta_{E_{c}}$ meets uniquely a unique critical circle $\gamma_{j}$ (in which case $\left(\delta_{E}\right)$ is called local); see Fig. 10;

- the end points $A_{j}(E)$ and $B_{j^{\prime}}(E)$ are one of those defined in Definition 2.5, and we will write $\delta_{E}=\left[A_{j}(E), B_{j^{\prime}}(E)\right]$;

- $\delta_{E}$ is always transversal to the $S^{1}$-orbits.

Here again, the normal form of Theorem 1.13 proves the existence of such a family of paths near any critical circle $\gamma_{j}$.

The goal of this section is finally to investigate the behaviour of the function $E \rightarrow \int_{\delta_{E}} \lambda_{h}$ as $E$ tends to a critical value, and to relate it to the holonomy of the sheaf $\left(\overline{\mathfrak{L}}, G\left(E_{c}\right)\right)$. The previous case where $\delta_{E}$ was a loop can always be recovered by composing paths of the type of Definition 2.6. Moreover, the regular theory implies that the so-called "local" paths can indeed be restricted to paths that are local in small neighbourhoods of the critical circles, since the following proposition holds:

Proposition 2.16. - If $\delta_{E}=\left[B_{j}(E), A_{j^{\prime}}(E)\right]$ is a regular family of paths (in the sense of Definition 2.6), then $E \rightarrow \int_{\delta_{E}} \lambda_{h}$ is smooth in $D^{ \pm}$and admits an asymptotic expansion in $h^{-1} C^{\infty}\left(D^{ \pm}\right) \llbracket h \rrbracket$. The first terms of this expansion are the following:

$$
\int_{\delta_{E}} \lambda_{h}=\Phi_{B_{j}}\left(B_{j}\right)-\Phi_{A_{j^{\prime}}}\left(A_{j^{\prime}}\right)+\frac{1}{h} \int_{\delta_{E}} \alpha+\int_{\delta_{E}} \kappa+\mu\left(\delta_{E}\right) \frac{\pi}{2}+\mathrm{O}(h),
$$

where $\Phi_{B_{j}}$ (resp. $\Phi_{A_{j^{\prime}}}$ ) is the phase of the principal symbol (viewed as a section of the KellerMaslov bundle over the Lagrangian manifold $\Lambda_{E} \backslash \Gamma_{j}-$ see e.g. [13] or [4]) of the fixed solution $u_{B_{j}}\left(\right.$ resp. $\left.u_{A_{j^{\prime}}}\right)$.

To study the neighbourhood of a critical circle, we shall use Theorem 1.5 to generalise the semiclassical invariant $\varepsilon_{2, j}(h)$ of Eq. (17) in a better way than Proposition 2.12 would do. That theorem still holds if $H_{1}$ and $H_{2}$ are replaced by $H_{1}-E_{1}$ and $H_{2}-E_{2}$, for a parameter $E=\left(E_{1}, E_{2}\right)$ varying near $\left(a_{o}, b_{o}\right)$. If we fix a critical circle $\gamma_{j}$ and $m \in \gamma_{j}$, the theorem yields a canonical change of coordinates $(x, y, \xi, \eta)$ near $m$, depending smoothly on $E$, and a function 
$\Phi_{E} \in C^{\infty}\left(\mathbb{R}^{2}, 0\right)$ depending smoothly on $E$, such that

$$
H_{1}-E_{1}=\xi, \quad H_{2}-E_{2}=\Phi_{E}(\xi, y \eta)
$$

(We have still $\partial_{2} \Phi_{E}(0,0)>0$.) This leads to yet another semiclassical normal form:

PROPOSITION 2.17. - Let $\gamma_{j}$ be a critical circle and $m \in \gamma_{j}$. There exists a microlocally unitary Fourier integral operator $U(h)$ associated to the canonical coordinates $(x, y, \xi, \eta)$, elliptic pseudodifferential operators $M_{1}(h), M_{2}(h)$ commuting (modulo $\mathrm{O}\left(h^{\infty}\right)$ ) with $Q_{1}$ and $Q_{2}$, and a real-valued function of $h$ (independent on $m, U, M_{i}$ ):

$$
\mathfrak{e}_{2}=\mathfrak{e}_{2, j}(E ; h) \sim \sum_{\ell=0}^{\infty} \mathfrak{e}_{2, j}^{(\ell)}(E) h^{\ell},
$$

such that, microlocally near $m$ :

$$
\begin{aligned}
& U^{-1}\left(\widehat{H}_{1}-E_{1}\right) U=Q_{1}+\mathrm{O}\left(h^{\infty}\right), \\
& U^{-1}\left(\widehat{H}_{2}-E_{2}\right) U=M_{1} Q_{1}+M_{2} \cdot\left(Q_{2}-\mathfrak{e}_{2}\right)+\mathrm{O}\left(h^{\infty}\right) .
\end{aligned}
$$

$M_{i}, U$ and $\mathfrak{e}_{2}$ depend smoothly on $E$.

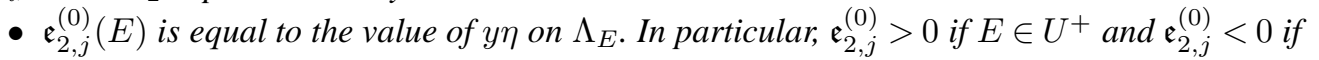
$E \in U^{-}$.

- If $E=E_{c} \in \mathcal{C}_{c}$,

$$
\mathfrak{e}_{2, j}^{(1)}(E)=\left(\frac{\lambda r_{1}-r_{2}}{\left|\mathcal{H}_{\Sigma}\left(H_{2}\right)\right|^{1 / 2}}\right)_{\mid \Gamma_{j} \cap \Lambda_{E}},
$$

where $r_{i}$ is the subprincipal symbol of $\widehat{H}_{i}, \lambda=\lambda\left(E_{c}\right)$ is the unique real number such that $H_{2}-\lambda H_{1}$ is critical on $\Gamma_{j} \cap \Lambda_{E}$ (see Lemma 1.7), and $\left|\mathcal{H}_{\Sigma}\left(H_{2}\right)\right|$ is the absolute value of the determinant of the transversal Hessian of $\mathrm{H}_{2}$. Note also that this denominator is equal to $\partial_{2} \Phi_{E}(0,0)$, and $\lambda=\partial_{1} \Phi_{E}(0,0)$.

Remark 2.11 (see Remark 2.10). - If $E$ is restricted to a domain of the form

$$
E=\left(a_{o}, b_{o}\right)+h\left(e_{1}, e_{2}\right)
$$

where $\left(a_{o}, b_{o}\right) \in \mathcal{C}_{c}$, then Theorem 2.1 applies with $e_{1}$ and $e_{2}$ as parameters, and yields an invariant $\varepsilon_{2, j}$, which can be recovered from $\mathfrak{e}_{2, j}$ by the following formula:

$$
\varepsilon_{2, j}\left(e_{1}, e_{2}\right)=\frac{1}{h} \mathfrak{e}_{2, j}\left(\left(a_{o}, b_{o}\right)+h\left(e_{1}, e_{2}\right)\right)+\mathrm{O}\left(h^{\infty}\right),
$$

or merely by viewing $-\left(e_{1}, e_{2}\right)$ as a correction of the subprincipal symbols and applying the formulae of the proposition. This proves the claim (12) of Theorem 2.1.

Using this proposition, we let $\beta=\frac{1}{2}+i \mathfrak{e}_{2} / h$ and $\zeta_{j}^{ \pm}$be the $h$-dependent functions in $C^{\infty}(U)$ defined by

$$
\begin{aligned}
\zeta_{j}^{+} & :=\frac{1}{\sqrt{2 \pi h}} \Gamma(\beta) \mathrm{e}^{\beta \ln h} \mathrm{e}^{-i \beta \frac{\pi}{2}}=\mathrm{e}^{-i \frac{\pi}{4}} \mathcal{E}_{j}\left(\mathfrak{e}_{2, j} / h\right) ; \\
\zeta_{j}^{-} & :=\frac{1}{\sqrt{2 \pi h}} \Gamma(\beta) \mathrm{e}^{\beta \ln h} \mathrm{e}^{i \beta \frac{\pi}{2}}=\mathrm{e}^{i \frac{\pi}{4}} \mathrm{e}^{-\mathfrak{e}_{2, j} \pi / h} \mathcal{E}_{j}\left(\mathfrak{e}_{2, j} / h\right) .
\end{aligned}
$$


$\left(\mathcal{E}_{j}\right.$ was defined in (20).) Next lemma, which directly follows from Stirling's formula, will be crucial for our analysis.

LEMMA 2.18. - For any $E \in U^{ \pm}$,

$$
\frac{1}{i} \ln \zeta_{j}^{ \pm}=\frac{1}{h}\left(\mathfrak{e}_{2, j}^{(0)} \ln \left|\mathfrak{e}_{2, j}^{(0)}\right|-\mathfrak{e}_{2, j}^{(0)}\right)+\mathfrak{e}_{2, j}^{(1)} \ln \left|\mathfrak{e}_{2, j}^{(0)}\right| \mp \frac{\pi}{4}+\mathrm{O}_{E}(h) .
$$

THEOREM 2.19. - Fix a component $k^{ \pm}$, and let $\delta_{E}=\left[A_{j}(E), B_{j}(E)\right]$ be a local path near the critical component $\Gamma_{j}$ (see Definition 2.6). Assume moreover that $\delta_{E}$ is oriented according to the flow of $H_{q}$ (otherwise just take the opposite of the holonomy!). Then there exists an $h$-dependent $\mathbb{R} / 2 \pi \mathbb{Z}$-valued function $g_{\delta}(h): E \rightarrow g_{\delta_{E}}(h) \in C^{\infty}\left(D^{ \pm}\right)$admitting a uniform asymptotic expansion of the form

$$
g_{\delta}(E ; h) \sim \sum_{\ell=-1}^{\infty} g_{\delta}^{(\ell)}(E) h^{\ell}, \quad g_{\delta}^{(\ell)} \in C^{\infty}\left(D^{ \pm}\right),
$$

such that

$$
\forall E \in U^{ \pm}, \quad g_{\delta}(E ; h)=\int_{\delta_{E}} \lambda_{h}-i \ln \left(\zeta_{j}^{ \pm}(E)\right) \quad(\bmod 2 \pi \mathbb{Z}) .
$$

The principal terms of $g_{\delta}(h)$ are given by the following formulae, for $E \in U^{ \pm}$:

$$
\begin{aligned}
& g_{\delta}^{(-1)}(E)=\int_{\delta_{E}} \alpha+\left(\mathfrak{e}_{2, j}^{(0)} \ln \left|\mathfrak{e}_{2, j}^{(0)}\right|-\mathfrak{e}_{2, j}^{(0)}\right)+\Phi_{A_{j}}^{(-1)}\left(A_{j}\right)-\Phi_{B_{j}}^{(-1)}\left(B_{j}\right) \\
& g_{\delta}^{(0)}(E)=\int_{\delta_{E}} \kappa_{E}+\mu\left(\delta_{E}\right) \frac{\pi}{2}+\left(\mp \frac{\pi}{4}+\mathfrak{e}_{2, j}^{(1)} \ln \left|\mathfrak{e}_{2, j}^{(0)}\right|\right)+\Phi_{A_{j}}^{(0)}\left(A_{j}\right)-\Phi_{B_{j}}^{(0)}\left(B_{j}\right),
\end{aligned}
$$

where $\Phi_{A_{j}}=\frac{1}{h} \Phi_{A_{j}}^{(-1)}+\Phi_{A_{j}}^{(0)}$ is the phase of the principal symbol of the fixed solution $u_{A_{j}}$ (and similarly for $u_{B_{j}}$ ).

THEOREM 2.20. - Let $E_{c}=\left(a_{o}, b_{o}\right) \in \mathcal{C}_{c}$. Let $\tilde{\delta}_{E_{c}}$ be a loop in $\Lambda_{E_{c}}$ oriented according to the flow of $H_{q}$ and of the form $\tilde{\delta}_{E_{c}}=\delta_{1}^{\text {loc }} \cdot \delta_{1}^{\text {reg }} \cdot \delta_{2}^{\text {loc }} \cdot \delta_{2}^{\text {reg }} \cdots \delta_{q}^{\text {loc }} \cdot \delta_{q}^{\text {reg }}$, where $\delta_{\ell}^{\text {loc }}$ and $\delta_{\ell}^{\text {reg }}$ are respectively "local" and "regular" paths in the sense of Definition 2.6. (The components $k^{ \pm}$ used for these paths may vary - see e.g. Fig 9.) Let

$$
g\left(E_{c} ; h\right) \sim \sum_{\ell=-1}^{\infty} g^{(\ell)}\left(E_{c}\right) h^{\ell}, \quad g^{(\ell)} \in C^{\infty}\left(\mathcal{C}_{c}\right)
$$

be defined as the sum

$$
g\left(E_{c} ; h\right):=\left(g_{\delta_{1}^{\text {loc }}}+g_{\delta_{1}^{\text {reg }}}+\cdots+g_{\delta_{q}^{\text {loc }}}+g_{\delta_{q}^{\text {reg }}}^{\text {reg }}\right)_{\uparrow E=E_{c}},
$$

where $g_{\delta_{k}^{\text {loc }}}$ is given by Theorem 2.19 and $g_{\delta_{k}^{\text {reg }}} \stackrel{\text { def }}{=} \int_{\delta_{k}^{\text {reg }}} \lambda_{h}$ (see Proposition 2.16). Then, under the hypothesis of Section 2.3,

$$
\operatorname{hol}\left(\bar{\delta}_{E_{c}}\right):=\mathrm{e}^{i g\left(E_{c} ; h\right)} \mathrm{e}^{i \frac{\pi}{2} n\left(N_{2}^{-}-N_{2}^{+}\right)}+\mathrm{O}\left(h^{\infty}\right),
$$




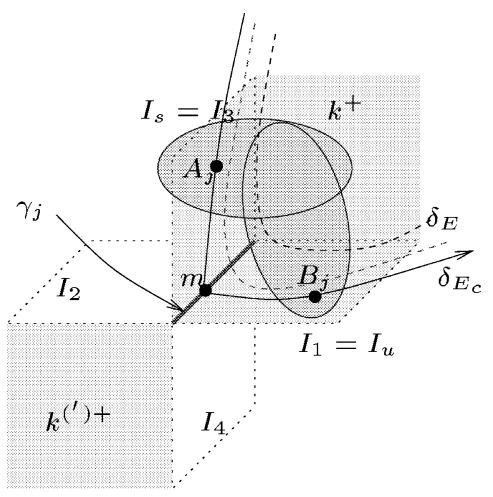

Fig. 11. The local holonomy.

where $\bar{\delta}_{E_{c}}$ is the projection of $\tilde{\delta}_{E_{c}}$ onto the graph $G\left(E_{c}\right)$ in the reduced orbifold $W\left(E_{c}\right), n$ is the "global quantum number" of Theorem 2.4, and $N_{2}^{ \pm}$is the number of local paths through a vertex of degree 2 and defined by a component $k \in N^{ \pm}$.

Proof of Theorem 2.19. - Fix a critical value $E_{c} \in \mathcal{C}_{c}$. Let $m$ be the intersection of the cycle $\delta_{E_{c}}$ with $\gamma_{j}$, and $\Omega_{m}$ an open set in which Proposition 2.17 applies. We can assume that the paths $\delta_{E}, E \in D^{ \pm}$all entirely lie in $\Omega_{m}$ (using Proposition 2.16, this will only modify $\int_{\delta_{E}} \lambda_{h}$ by an additive term entering in $g_{\delta}(E ; h)$ ). As before, label the local un/stable manifolds with cyclic order $I_{1}, I_{3}, I_{2}$ and $I_{4} . \delta_{E_{c}}$ enters $\Omega_{m}$ on a local stable manifold $I_{s}, s=3,4$, and leaves it on a local unstable manifold $I_{u}, u=1,2$ ( $u$ is the index preceding $s$ in the cycle $(1,3,2,4)$ if $k^{ \pm}=k^{+} \in N^{+}$and the index following $s$ if $\left.k^{ \pm}=k^{-} \in N^{-}\right)$. As before, we endow a neighbourhood of each $I_{\alpha}$ with the distribution $e_{\alpha}$ :

$$
\begin{aligned}
& e_{1}:=u_{+}^{\mathfrak{e}_{2, j} / h}:=1_{ \pm y>0} \frac{1}{\sqrt{|y|}} \mathrm{e}^{i \mathfrak{e}_{2, j} \ln |y| / h}, \\
& e_{2}:=u_{-}^{\mathfrak{e}_{2, j} / h}:=1_{ \pm y<0} \frac{1}{\sqrt{|y|}} \mathrm{e}^{i \mathfrak{e}_{2, j} \ln |y| / h}, \\
& e_{3}:=\mathcal{F}_{h}^{-1}\left(u_{+}^{-\mathfrak{e}_{2, j} / h}\right), \\
& e_{4}:=\mathcal{F}_{h}^{-1}\left(u_{-}^{-\mathfrak{e}_{2, j} / h}\right) .
\end{aligned}
$$

These distributions are classical Lagrangian distributions whose phases admit an asymptotic expansion in $\frac{1}{h} C^{\infty}\left(D^{ \pm}\right) \llbracket h \rrbracket$. Moreover, they are microlocal solutions of (26) in $\Omega_{m}$ uniformly for $E \in D^{ \pm}$, and hence constitute an admissible choice in view of Definition 2.5. Note that this choice possibly implies another additive term entering in $g_{\delta_{E}}$. In a small ball around $A_{j}\left(E_{c}\right)$, the space of solutions has dimension 1 and we must have a constant $C^{ \pm}(E, h)$ such that $e_{u} \sim C^{ \pm}(E, h) e_{s}$. Now Definition 2.5 says that, with respect to the fixed solutions $e_{u}$ and $e_{s}$,

$$
C^{ \pm}(E, h)=\mathrm{e}^{-i \int_{\delta_{E}} \lambda_{h}} \quad\left(\bmod \mathrm{O}\left(h^{\infty}\right)\right)
$$

The expression of the Fourier transform involved in $e_{s}$ shows - as in [8] - that

$$
C^{ \pm}(E, h)=\zeta_{j}^{ \pm} \quad\left(\bmod \mathrm{O}\left(h^{\infty}\right)\right)
$$


This proves the existence of the claimed $g_{\delta}$ satisfying Eq. (35). The formulae (36) and (37) now follow from Stirling's formula (Lemma 2.18) and from the fact that the asymptotic expansion for $g_{\delta}$ is uniform in $D^{ \pm}$, and hence is given for any fixed $E \in U^{ \pm}$by the difference of the (non-uniform) asymptotic expansions of $\int_{\delta_{E}} \lambda_{h}$ and $i \ln \left(\zeta_{j}^{ \pm}(E)\right.$ ). We have also used the formula (31) which holds only for non-critical $E$, and which comes from the definition of the bundle of principal symbols.

Note that we do not see here any difference between critical circles of direct/reverse type, for the statement is purely local near a point of $\gamma_{j}$.

Proof of Theorem 2.20. - To each $\delta_{\ell}^{\text {loc }}$ are associated a unique component $k^{s_{\ell}}, s_{\ell}= \pm$, a unique critical circle $\gamma_{j_{\ell}}$, and a (non-unique) local family of paths $\delta_{\ell}^{\text {loc }}(E)$ (in the sense of Definition 2.6) such that $\delta_{\ell}^{\text {loc }}=\delta_{\ell}^{\text {loc }}\left(E_{c}\right)$. Let $\mathcal{I}_{h}^{s_{\ell}}$ be the set of $E \in D^{s_{\ell}}$ such that $\left(\mathfrak{e}_{1}\right)_{\uparrow k^{s_{\ell}} \in} \in$ $h \mathbb{Z}+\mathrm{O}\left(h^{\infty}\right)$ (i.e. $\mathcal{I}_{h}^{s_{\ell}}=\varpi_{h}^{-1}\left[h \mathbb{Z}+\mathrm{O}\left(h^{\infty}\right)\right]$ in the notation of the proof of Corollary 2.14). Then the (assumed) hypothesis of Section 2.3 " $\varepsilon_{1}=n$ " says that $E_{c} \in \mathcal{I}_{h}^{s \ell}$. Using Proposition 2.12 one can construct smooth families $\left(u_{E}\right)_{E \in \mathcal{I}_{h}^{s_{\ell}}}^{1,2,4}$ of solutions on a neighbourhood of $\gamma_{j \ell}$ in $\overline{k^{s_{\ell}}}$ such that

$$
\left(u_{E}^{1}, u_{E}^{2}, u_{E}^{3}, u_{E}^{4}\right)
$$

- in the direct case - or

$$
\left\{\begin{array}{l}
u_{E}^{1}+(-1)^{n} u_{E}^{2} \\
\mathrm{e}^{i \frac{\pi}{2} n} u_{E}^{3}+\mathrm{e}^{-i \frac{\pi}{2} n} u_{E}^{4}
\end{array}\right.
$$

- in the reverse case - form at $E=E_{c}$ a standard basis for the graph $G\left(E_{c}\right)$ at the vertex $g_{j_{\ell}}$.

Since these solutions are smooth WKB solutions and hence admissible in the sense of Definition 2.5, we shall use them to define the local holonomies $\int_{\delta_{\ell}^{\text {loc } / \mathrm{reg}}(E)} \lambda_{h}$; since they are standard basis at $E=E_{c}$, we shall in the same way use them to define the local "reduced" holonomies $\operatorname{hol}\left(\bar{\delta}_{\ell}^{\text {loc/reg }}\right)$. But then by definition of the sheaf $\left(\overline{\mathfrak{L}}, G\left(E_{c}\right)\right)$ we have

$$
\left\{\begin{array}{l}
\operatorname{hol}\left(\bar{\delta}_{\ell}^{\text {loc }}\right)=1 \text { and } \\
\operatorname{hol}\left(\bar{\delta}_{\ell}^{\text {reg }}\right)=\exp \left(i \int_{\delta_{\ell}^{\text {reg }}\left(E_{c}\right)} \lambda_{h}\right)=\exp \left(i g_{\delta_{\ell}^{\text {reg }}}\left(E_{c}\right)\right) .
\end{array}\right.
$$

On the other hand, we know from the proof of Theorem 2.19 that for such a choice of microlocal solutions, we have (modulo $2 \pi$ )

$$
\begin{cases}g_{\delta_{\ell}^{\text {loc }}}(E)=0 & \text { if } \gamma_{j_{\ell}} \text { is of degree } 4 \\ g_{\delta_{\ell}^{\text {loc }}}(E)=s_{\ell} \frac{\pi}{2} n & \text { if } \gamma_{j_{\ell}} \text { is of degree } 2\end{cases}
$$

Therefore, if we decompose

$$
\operatorname{hol}\left(\bar{\delta}_{E_{c}}\right)=\prod_{\ell / \operatorname{deg} \gamma_{j_{\ell}}=2} \operatorname{hol}\left(\bar{\delta}_{\ell}^{\mathrm{loc}}\right) \times \prod_{\ell / \operatorname{deg} \gamma_{j_{\ell}}=4} \operatorname{hol}\left(\bar{\delta}_{\ell}^{\mathrm{loc}}\right) \times \prod_{\ell} \operatorname{hol}\left(\bar{\delta}_{\ell}^{\mathrm{reg}}\right),
$$

we obtain by (42) and (43):

$$
\operatorname{hol}\left(\bar{\delta}_{E_{c}}\right)=\exp \left(i \sum_{\ell / \operatorname{deg} \gamma_{j_{\ell}}=2}\left(g_{\delta_{\ell}^{\operatorname{loc}}}\left(E_{c}\right)-s_{\ell} \frac{\pi}{2} n\right)+\sum_{\ell / \operatorname{deg} \gamma_{j_{\ell}}=4} g_{\delta_{\ell}^{\operatorname{loc}}}\left(E_{c}\right)+\sum_{\ell} g_{\delta_{\ell}^{\text {reg }}}\left(E_{c}\right)\right),
$$

which proves the theorem. 
COROLLARY 2.21. - Theorem 2.20 together with formulae (36) and (37) finally prove the second point of Proposition 2.6.

Proof. -

1. The principal action. Since $\mathfrak{e}_{2, j}^{(0)}\left(E_{c}\right)=0$, it is clear from (36) that for any $\ell=1, \ldots, q$,

$$
g_{\delta_{\ell}^{\text {loc }}}^{(-1)}\left(E_{c}\right)=\int_{\delta_{\ell}^{\text {loc }}} \alpha+\Phi_{A_{j_{\ell}}}^{(-1)}\left(A_{j_{\ell}}\left(E_{c}\right)\right)-\Phi_{B_{j_{\ell}}}^{(-1)}\left(B_{j_{\ell}}\left(E_{c}\right)\right)
$$

and from (31) that for any $\ell=1, \ldots, q$ (identifying $\ell=q+1$ with $\ell=1$ ),

$$
g_{\delta_{\ell}^{\text {reg }}}^{(-1)}\left(E_{c}\right)=\int_{\delta_{\ell}^{\text {reg }}} \alpha+\Phi_{B_{j_{\ell}}}^{(-1)}\left(B_{j_{\ell}}\left(E_{c}\right)\right)-\Phi_{A_{j_{\ell+1}}}^{(-1)}\left(B_{j_{\ell+1}}\left(E_{c}\right)\right) .
$$

Therefore,

$$
g^{(-1)}\left(E_{c}\right)=\int_{\tilde{\delta}_{E_{c}}} \alpha .
$$

2. The sub-principal action and the Maslov index. Fix $\ell=1, \ldots, q$ and let $\gamma_{j}=\gamma_{j_{\ell}}$. Let $m=m\left(E_{c}\right)$ be the point where $\delta_{\ell}^{\text {loc }}$ meets $\gamma_{j}$. As in Proposition 2.17, we shall use the local canonical coordinates at $m$ given by Theorem 1.5.

Recalling the notation of Proposition 2.17, (32) implies that

$$
\left(\mathcal{X}_{1}, \mathcal{X}_{2}\right)=\left(\begin{array}{cc}
1 & 0 \\
\partial_{1} \Phi_{E} & \partial_{2} \Phi_{E}
\end{array}\right) \cdot\left(\mathcal{X}_{\xi}, \mathcal{X}_{y \eta}\right)
$$

Since $\partial_{2} \Phi_{E} \neq 0$, there exists a smooth function $\rho_{2}=\frac{-\partial_{1} \Phi_{E} r_{1}+r_{2}}{\partial_{2} \Phi_{E}}$ (depending also smoothly on $E$ ) such that the subprincipal form $\kappa_{E}$ is given by

$$
\kappa_{E} \cdot\left(\mathcal{X}_{\xi}, \mathcal{X}_{y \eta}\right)=-\left(r_{1}, \rho_{2}\right)
$$

Note that for a critical value $E=E_{c},\left(\rho_{2}\right)_{\mid y=\eta=0}=-\mathfrak{e}_{2, j}^{(1)}$. The closedness of $\kappa_{E}$ on each $\Lambda_{E}$ implies that

$$
\left\{r_{1}, y \eta\right\}=\left\{\rho_{2}, \xi\right\} .
$$

Using a local analogue of Lemma 2.3, we can decompose $\left(r_{1}, \rho_{2}\right)$ in the following way:

$$
\left(r_{1}, \rho_{2}\right)=(0, K)-\left(\left\{\mathcal{X}_{\xi}, \tilde{f}\right\},\left\{\mathcal{X}_{y \eta}, \tilde{f}\right\}\right)
$$

for some smooth functions $\tilde{f}, K$ where $K$ commutes with $y \eta$ and $\xi$. Therefore the function

$$
f:=\tilde{f}-K \ln |y| \quad(\text { or } \tilde{f}+K \ln |\eta| \text { where } y=0),
$$

restricted to $\Lambda_{E}$, satisfies $d_{\Lambda_{E}} \tilde{f}=\kappa_{E}$. We can now compute

$$
\int_{\delta_{\ell}^{\text {loc }}} \kappa_{E}=f\left(B_{j}\right)-f\left(A_{j}\right)=\tilde{f}\left(B_{j}\right)-\tilde{f}\left(A_{j}\right)-K \ln \left|y_{B_{j}} \eta_{A_{j}}\right|+K \ln \left|y_{A_{j}} \eta_{A_{j}}\right| .
$$


Since $A_{j}(E)$ and $B_{j}(E)$ are in $\Lambda_{E}, y_{A_{j}} \eta_{A_{j}}=\mathfrak{e}_{2, j}^{(0)}$ and we have

$$
\int_{\delta_{\ell}^{\text {loc }}} \kappa_{E}+\mathfrak{e}_{2, j}^{(1)} \ln \left|\mathfrak{e}_{2, j}^{(0)}\right|=\tilde{f}\left(B_{j}\right)-\tilde{f}\left(A_{j}\right)-K \ln \left|y_{B_{j}} \eta_{A_{j}}\right|+\left(\mathfrak{e}_{2, j}^{(1)}+K\right) \ln \left|\mathfrak{e}_{2, j}^{(0)}\right| .
$$

Because of (44), $\rho_{2}-K$ vanish at $y=\eta=0$, hence $K=-\mathfrak{e}_{2, j}^{(1)}+\mathrm{O}(y \eta)$ and $\left(\mathfrak{e}_{2, j}^{(1)}+K\right) \ln \left|\mathfrak{e}_{2, j}^{(0)}\right|$ tends to zero as $E$ tends to a critical value $E_{c}$. Since $g_{\delta_{\ell}^{\text {loc }}}^{(0)}$ is smooth at $E_{c}$, the formula (37) implies that $\mu\left(\delta_{\ell}^{\operatorname{loc}}(E)\right)$ is continuous at $E_{c}$ and hence constant; let us denote it by $\mu\left(\delta_{\ell}^{\operatorname{loc}}\left(E_{c}\right)\right)$.

Suppose now that $E=E_{c}$ and let $a, b$ be points on $\delta_{\ell}^{\text {loc }}\left(E_{c}\right)$ located respectively in $\left[A_{j}, m\right]$ and $\left[m, B_{j}\right]$. Then

$$
\tilde{f}\left(B_{j}\right)-\tilde{f}\left(A_{j}\right)-K \ln \left|y_{B_{j}} \eta_{A_{j}}\right|=\lim _{a, b \rightarrow m}\left(\tilde{f}(a)-\tilde{f}\left(A_{j}\right)+\tilde{f}\left(B_{j}\right)-\tilde{f}(b)-K \ln \left|y_{B_{j}} \eta_{A_{j}}\right|\right) .
$$

The term in the limit is equal to

$$
\int_{\left[A_{j}, a\right]} \kappa_{E_{c}}+\int_{\left[b, B_{j}\right]} \kappa_{E_{c}}+\mathfrak{e}_{2, j}^{(1)} \ln \left|y_{b} \eta_{a}\right| .
$$

Therefore, by Definition 2.2 , the limit is equal to $\int_{\delta_{\ell}^{\text {loc }}} \tilde{\kappa}_{E_{c}}$, and (37) yields:

$$
g_{\delta_{\ell}^{\text {loc }}}^{(0)}\left(E_{c}\right)=\int_{\delta_{\ell}^{\text {loc }}} \tilde{\kappa}_{E_{c}}+\mu\left(\delta_{\ell}^{\text {loc }}\left(E_{c}\right)\right) \frac{\pi}{2}-s_{\ell} \frac{\pi}{4}+d \Phi_{A_{j}}^{(0)}\left(A_{j}\left(E_{c}\right)\right)-\Phi_{B_{j}}^{(0)}\left(B_{j}\left(E_{c}\right)\right) .
$$

Then as before, if we sum up all the contributions from regular and local paths, we obtain

$$
g^{(0)}\left(E_{c}\right)=\int_{\tilde{\delta}_{E_{c}}} \tilde{\kappa}_{E_{c}}+\mu\left(\tilde{\delta}_{E_{c}}\right) \frac{\pi}{2}+\sum_{\ell}-s_{\ell} \frac{\pi}{4} .
$$

Using Definition 2.2 of the regularized Maslov cocycle, we finally obtain

$$
g^{(0)}\left(E_{c}\right)+\frac{\pi}{2}\left(N_{2}^{-}-N_{2}^{+}\right)=\int_{\tilde{\delta}_{E_{c}}} \tilde{\kappa}_{E_{c}}+\tilde{\mu}\left(\tilde{\delta}_{E_{c}}\right) \frac{\pi}{2},
$$

and Eq. (38) concludes the proof.

\section{Examples}

We propose in this section several examples for which our theory applies. Many other could probably be found; the ones presented here are interesting by their simplicity and yet by their rich structure and behaviour.

\subsection{The recipe}

Let us recall here briefly the recipe for obtaining the semiclassical quantisation rules. The first thing to do is to locate the critical value of transversal hyperbolic type in the image of the 
momentum map $F=\left(H_{1}, H_{2}\right)$. Then choose one of these points $o$ and describe the singular level set $\Lambda_{o}=F^{-1}(o)$, in order to have: (a) the graph $G$, (b) a formula for the vector fields $\mathcal{X}_{1}$ and $\mathcal{X}_{2}$ on $\Lambda_{o}$ - and if it is not one of these, for the periodic vector field $\mathcal{X}_{p}$.

Compute the semiclassical invariants (action integral, Maslov index) for a periodic cycle - this implies only regular tools - in order to derive the first quantisation condition of Theorem 2.4 up to $\mathrm{O}(h)$, and fix the quantum number $n$.

From the graph, apply Theorem 2.7 to obtain the second quantisation rule in the form of a determinantal equation. It remains to compute the holonomy hol up to $\mathrm{O}(h)$, which involves the singular semiclassical invariants of Definition 2.2. The fulfillment of these quantisations rules determine the spectrum up to an error of order $\mathrm{O}\left(h^{2}\right)$ in a window of size $\mathrm{O}(h)$ (in fact, it is easy to determine the smooth dependence of the semiclassical invariants in $o$, and the spectral window can be extended to a rectangular domain of size $\mathrm{O}(1)$ along the curve of critical values, and of size $\mathrm{O}(h)$ in the transversal direction).

Notation. - The reader must be warned that the symbol $e$ (italic) is used as a subprincipal spectral parameter (as in " $E=h e$ "), while the exponential is denoted by $\mathrm{e}^{a}=\exp (a)$.

\subsection{Laplacians on ellipsoids}

The geodesic flow on the ellipsoid gives a natural example where our geometrical analysis applies and for which all objects are explicit. However we give no detail about the semiclassical treatment since a separation of variables shows that our formula reduces to two problems of one degree of freedom, which can be solved as in [10].

Let us consider the ellipsoid in the Euclidian space $\mathbb{R}^{3}$ defined by:

$$
E=\left\{\frac{x_{1}^{2}}{a_{1}^{2}}+\frac{x_{2}^{2}}{a_{2}^{2}}+\frac{x_{3}^{2}}{a_{3}^{2}}=1\right\}
$$

with $0<a_{1}<a_{2}<a_{3}$. The geodesic flow on $E$ has been discovered to be integrable by Jacobi in 1838 using Abelian integrals. For a recent presentation, one can read [21,2,24] or [22].

\subsubsection{Classics}

Let us denote by $P, Q, P^{\prime}=-P, Q^{\prime}=-Q$ the four umbilics of $E$ which are located on the ellipse $\left\{x_{2}=0\right\}$. If

$$
X_{1}=\sqrt{a_{1}\left(a_{2}-a_{1}\right)\left(a_{3}-a_{1}\right)} \quad \text { and } \quad X_{3}=\sqrt{a_{3}\left(a_{3}-a_{2}\right)\left(a_{3}-a_{1}\right)},
$$

we have

$$
P=\left(X_{1}, 0, X_{3}\right), \quad Q=\left(-X_{1}, 0, X_{3}\right) .
$$

We will consider the (unique up to global dilatation) conformal representation $\Phi$ of $E_{+}=E \cap\left\{x_{2}>0\right\}$ on a rectangle $\left.R=\right] 0, T_{1}[\times] 0, T_{2}[$ such that the four umbilics are going on the four vertices of $R$, according to Fig. 15. Using such coordinates $(x, y) \in R$, we get (see [12] (Vol. 2, p. 308 and Vol. 3, p. 13) or [20]) the following expression for the metric of $E$ :

$$
d s^{2}=\left(a^{2}(x)+b^{2}(y)\right)\left(d x^{2}+d y^{2}\right)
$$

where $a, b$ are given in terms of hyperelliptic integrals and extend to smooth functions on $\mathbb{R}$ which satisfy: $a$ is $>0$ on $] 0, T_{1}$ [, vanishes exactly at the points $k T_{1}, k \in \mathbb{Z}$, and is odd with respect to $T_{1} \mathbb{Z}$ and $b$ satisfies the same properties with respect to $T_{2}$. Moreover $a^{\prime}(0)=b^{\prime}(0)>0$. Let 


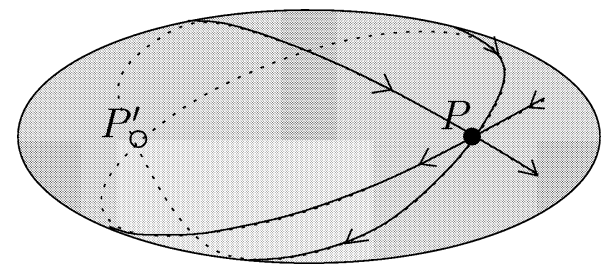

Fig. 12. A geodesic passing through $P$ and $P^{\prime}$.

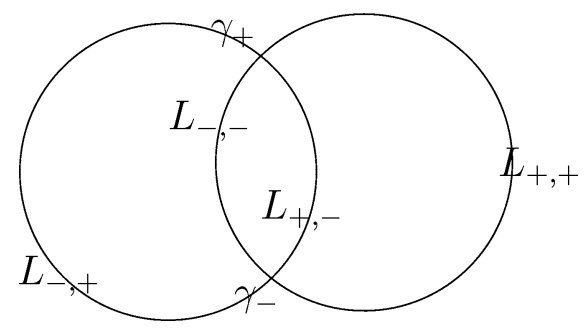

Fig. 13. Associated graph for $E$.

us denote by $\Gamma$ the lattice $T_{1} \mathbb{Z} \oplus T_{2} \mathbb{Z}$. Then $d s^{2}$ extends into a smooth metric on $\mathbb{R}^{2} \backslash \Gamma$, which is $\Gamma$-periodic. Let us consider the torus $T=\mathbb{R}^{2} / 2 \Gamma$. Then the map $\sigma: T \rightarrow T$ defined by $T(z)=-z$ defines an isometric involution of $T$ with four fixed points and we get a natural identification of $E$ with $T / \sigma$ as a 2-sheeted branched covering $\Pi$ of $T$ over $E$ with automorphism $\sigma$. The metric $d s^{2}$ admits conical singular points of total angle $4 \pi$ at the umbilics which makes the metric on $E$ smooth. More precisely, it follows from the formulae of [20] that there exists an analytic function $G$ defined near 0 , with $G(0)=0, G^{\prime}(0)>0$ such that near $(0,0)$ we have $d s^{2}=\left(G\left(x^{2}\right)-G\left(-y^{2}\right)\right)\left(d x^{2}+d y^{2}\right)$. It is rather easy to check that there exists an analytic function $A(u, v)$ with $A(0,0)>0$ such that $G\left(x^{2}\right)-G\left(-y^{2}\right)=\left(x^{2}+y^{2}\right) A\left(x^{2}-y^{2}, 2 x y\right)$ and, if locally $Z=\Pi(z)=z^{2}$, we have: $d s^{2}=\Pi^{\star}\left(4 A(X, Y)\left(d X^{2}+d Y^{2}\right)\right)$.

We will use the fundamental domain $D=\left[0,2 T_{1}\right] \times\left[0, T_{2}\right]$. $E$ can be recovered from this rectangle by gluing edges as indicated on Fig. 15.

If $a, b$ were non-vanishing, $d s^{2}$ would be called a Liouville metric on T. Our case corresponds to a degenerate Liouville metric on the sphere. It is well known that Liouville metrics are integrable. Let us denote by

$$
H_{1}=\frac{\xi^{2}+\eta^{2}}{a^{2}(x)+b^{2}(y)}
$$

the geodesic flow and by

$$
H_{2}=\frac{b^{2}(y) \xi^{2}-a^{2}(x) \eta^{2}}{a^{2}(x)+b^{2}(y)} .
$$

The manifold $L_{E, F}=\left\{H_{1}=E, H_{2}=F\right\}$ is given by:

$$
L_{E, F}=\left\{\xi^{2}=F+a^{2}(x) E, \eta^{2}=b^{2}(y) E-F\right\}
$$

which is obviously Lagrangian.

We are interested in the singular value $o=(E=1, F=0)$ of the moment map $\left(H_{1}, H_{2}\right)$ and the corresponding $\Lambda_{o}$. Geodesics passing through $P$ (resp. $Q$ ) contain also $P^{\prime}$ (resp. $Q^{\prime}$ ) and 


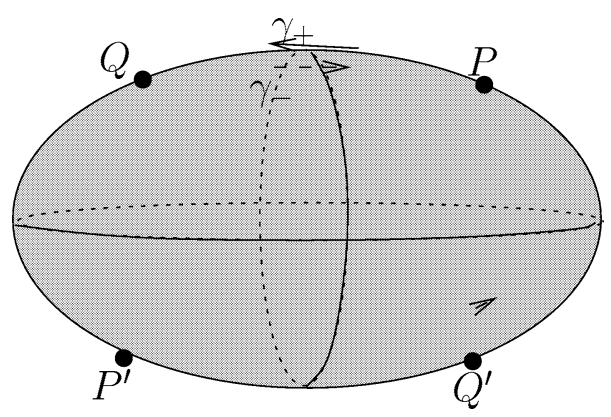

Fig. 14. The ellipsoid.

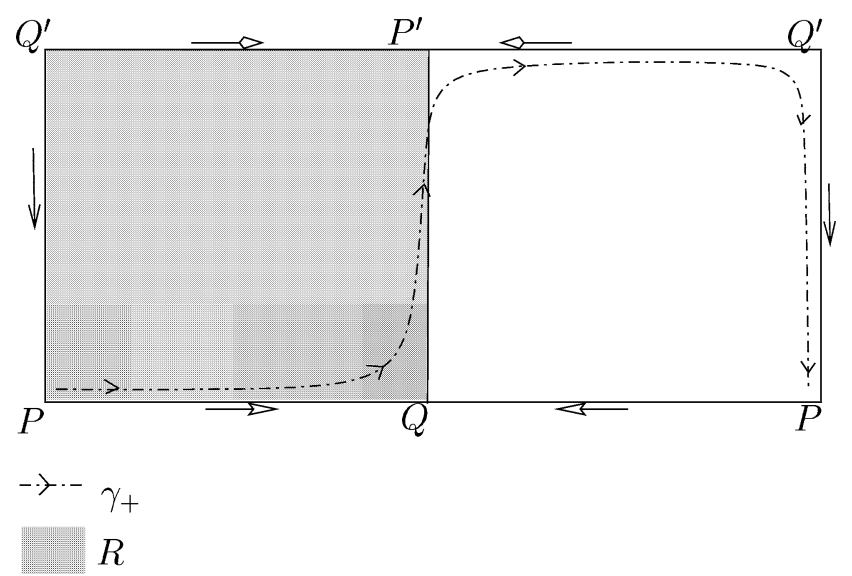

Fig. 15. Fundamental domain.

vice-versa. $\Lambda_{o}$ is the set of unit covectors corresponding to geodesics passing through $P$ or $Q$. We have $\Lambda_{o}=\bigcup L_{ \pm, \pm}$where $L_{ \pm, \pm}=\{\xi= \pm a(x), \eta= \pm b(y)\}$. In particular $L_{P}=L_{+,+} \cup L_{-,-}$ and $L_{Q}=L_{+,-} \cup L_{-,+}$are smooth Lagrangian tori whose intersection is $\gamma_{+} \cup \gamma_{-}$. Here $\gamma_{+}$ (resp. $\left.\gamma_{-}\right)$is the lift of the ellipse $x_{2}=0$ with orientation $\left(P, Q, P^{\prime}, Q^{\prime}\right)$ (resp. opposite). $L_{P}$ (resp. $L_{Q}$ ) is the set of unit covectors of geodesics of $E$ containing $P$ and $P^{\prime}$ (resp. $Q$ and $Q^{\prime}$ ). $L_{P}$ (resp. $L_{Q}$ ) is the stable manifold of $\gamma_{-}$(resp. $\gamma_{+}$) and the unstable manifold of $\gamma_{+}$(resp. $\left.\gamma_{-}\right)$.

The associated graph $G$ is the union of 2 circles corresponding to $L_{P}$ and $L_{Q}$ intersecting at 2 points corresponding to $\gamma_{ \pm}$.

\subsubsection{Quantum}

We now introduce the quantum Hamiltonian $\widehat{H}_{1}=h^{2} \Delta_{E}$ which is given in the coordinates $(x, y)$ by:

$$
\widehat{H}_{1}=-\frac{h^{2}}{a^{2}+b^{2}}\left(\partial_{x}^{2}+\partial_{y}^{2}\right)
$$

and the operator

$$
\widehat{H}_{2}=-\frac{h^{2}}{a^{2}+b^{2}}\left(b^{2} \partial_{x}^{2}-a^{2} \partial_{y}^{2}\right) .
$$


It is possible to check directly that $\left[\widehat{H}_{1}, \widehat{H}_{2}\right]=0$ outside of the lattice $\Gamma$ which is the set of singular points. We need a stronger form of commutation, namely if

$$
\widehat{H}_{1} \varphi=\lambda \varphi,
$$

we need to prove that $\widehat{H}_{2} \varphi$ is in the domain of $\widehat{H}_{1}$ and $\widehat{H}_{1}\left(\widehat{H}_{2} \varphi\right)=\lambda \widehat{H}_{2} \varphi$, so that $\widehat{H}_{1}$ and $\widehat{H}_{2}$ have a commun eigenbasis.

For that, we need a characterisation of the functions $\tilde{\varphi}=\varphi \circ \Pi$ where $\varphi$ is an eigenfunction of the Riemannian Laplacian $\Delta_{E}$ on $E$.

LEMMA 3.1. $-\tilde{\varphi}: T \rightarrow \mathbb{C}$ is of the form $\tilde{\varphi}=\varphi \circ \Pi$ with $\Delta_{E} \varphi=\lambda \varphi$ if and only if $\tilde{\varphi}$ is $\sigma$ invariant and satifies

$$
-\tilde{\varphi}_{x^{2}}^{\prime \prime}-\tilde{\varphi}_{y^{2}}^{\prime \prime}=\lambda\left(a^{2}+b^{2}\right) \tilde{\varphi} .
$$

Proof. - Starting from $\varphi$ an eigenfunction of $\Delta_{E}$ and using smoothness of $\Pi$ gives the trivial direction. For the other, using the fact that $\tilde{\varphi} \circ \sigma=\tilde{\varphi}$, we get a bounded $\varphi$ with $\tilde{\varphi}=\varphi \circ \Pi$ and $\left(\Delta_{E}-\lambda\right) \varphi=T$ where $T$ is supported inside the (finite) set of umbilics. We deduce that $T$ is 0 or that $\varphi$ (and hence $\tilde{\varphi}$ ) is unbounded.

It is easy to check that $\widehat{H}_{2} \tilde{\varphi}$ is smooth using Eq. (46). Then $\widehat{H}_{2} \tilde{\varphi}$ satisfies Eq. (47) outside $\Gamma$ and hence everywhere on $T$ and we get a commun eigenbasis for $\widehat{H}_{1}$ and $\widehat{H}_{2}$.

We prefer to rewrite the eigenvectors equations

$$
\widehat{H}_{1} \tilde{\varphi}=\lambda \tilde{\varphi}, \quad \widehat{H}_{2} \tilde{\varphi}=\mu \tilde{\varphi}
$$

in the following simpler way:

$$
\begin{aligned}
& \widehat{P} \tilde{\varphi}:=h^{2} \frac{\partial^{2} \tilde{\varphi}}{\partial x^{2}}+\left(a^{2}(x) \lambda+\mu\right) \tilde{\varphi}=0, \\
& \widehat{Q} \tilde{\varphi}:=h^{2} \frac{\partial^{2} \tilde{\varphi}}{\partial y^{2}}+\left(b^{2}(y) \lambda-\mu\right) \tilde{\varphi}=0 .
\end{aligned}
$$

We are interested in solutions of this system which are $\sigma$ invariant. If we denote by $\sigma_{1}(x, y)=(-x, y)$ and $\sigma_{2}(x, y)=(x,-y)$, we get $\sigma=\sigma_{1} \circ \sigma_{2}$ and because $\sigma_{j}$ commutes with $\widehat{P}$ and $\widehat{Q}$ we are reduced to find solutions of the form $\tilde{\varphi}(x, y)=f(x) g(y)$ with $f$ a $2 T_{1}$-periodic solution of $\widehat{P} f=0$ and $g$ a $2 T_{2}$-periodic solution of $\widehat{Q} f=0$. We ask moreover that $f$ and $g$ are both even or both odd. We assume $\lambda=1$ which corresponds to quantize $h$ and $\mu=\varepsilon h$. The associated fiber of the momentum map is then $\Lambda_{o}$.

This way we are reduced to 2 one-dimensional problems and because $\widehat{P}$ and $\widehat{Q}$ are semiclassical stationary Schrödinger operators with potentials $-a^{2}$ and $-b^{2}$, we are reduced to the computations of [10, pp. 489-490] for periodic double wells.

\section{3. $1: 2$-resonance}

\subsubsection{Birkhoff normal forms}

Consider a Hamiltonian $H: T^{*} \mathbb{R}^{2} \rightarrow \mathbb{R}$ with a non-degenerate minimum at the origin. We can assume using a symplectic linear change that $H\left(z_{1}, z_{2}\right)=K_{2}(z)+\mathrm{O}\left(|z|^{3}\right)$ with

$$
K_{2}(z)=\omega_{1}\left|z_{1}\right|^{2}+\omega_{2}\left|z_{2}\right|^{2}
$$

and $\omega_{j}>0$. Here $z_{j}=x_{j}+i \xi_{j}$, where $\left(x_{1}, x_{2}, \xi_{1}, \xi_{2}\right)$ are canonical coordinates for $T^{*} \mathbb{R}^{2}$. We will say that the quadratic part is resonant if $\omega_{1} / \omega_{2}$ is a rational number. It is possible to derive a 
Birkhoff normal form $H$ of the following form

$$
H=K_{2}+R+\mathrm{O}(|z|)^{\infty}
$$

with $R=\mathrm{O}\left(|z|^{3}\right)$ and $\left\{K_{2}, R\right\}=0$. The same result is true on the quantum level (see Chapter 5 of [26]) with commuting operators $\widehat{K}_{2}$ and $\widehat{R}$. If we are able to analyse the joint spectrum of the operators $\widehat{K}_{2}, \widehat{R}$ we can deduce some sharp results for eigenstates in the energy domain $E=\mathrm{O}\left(h^{\alpha}\right)$ with $\alpha>0$. In the case of the $1: 1$ resonance -i.e. $\omega_{1}=\omega_{2}$-the flow of $K_{2}$ induces a free circle action on the energy hypersurface $K_{2}=$ const and the reduced space is smooth. Then, via the use of Toeplitz operators, the problem is fully reduced to a 1-dimensional one. This is no longer the case for the $1: 2$ resonance, where the reduced phase space has a conical singularity. For this simple example, we will show that our analysis applies. Another application would be the near $1: 2$ resonance with $R=\varepsilon\left|z_{1}\right|^{2}+R^{\prime}$.

\subsection{2. $1: 2$ resonance}

We consider the following Poisson commuting Hamiltonians on $T^{\star} \mathbb{R}^{2}$ :

$$
H_{1}=\frac{1}{2}\left|z_{1}\right|^{2}+\left|z_{2}\right|^{2}, \quad H_{2}=\left(x_{1}^{2}-\xi_{1}^{2}\right) x_{2}+2 x_{1} \xi_{1} \xi_{2}=\Re\left(z_{1}^{2} \bar{z}_{2}\right)
$$

with $z_{j}=x_{j}+i \xi_{j}, j=1,2$. The image of the momentum map $F=\left(H_{1}, H_{2}\right)$ is

$$
F\left(T^{*} \mathbb{R}^{2}\right)=\left\{(X, Y) \mid 16 X^{3} \geqslant 27 Y^{2}\right\} .
$$

The singular values consist of the boundary (which corresponds to transversal elliptic points, except for the origin which is degenerate) and the half line $\mathcal{C}_{c}=\{(X, 0), X>0\}$, whose points are transversally hyperbolic (see Fig. 16). Here we shall be interested in the critical values on $\mathcal{C}_{c}$. Because of the homogeneity of $H_{j}$, it is sufficient to consider the point $o=(1,0)$.

The corresponding commuting quantum Hamiltonians are:

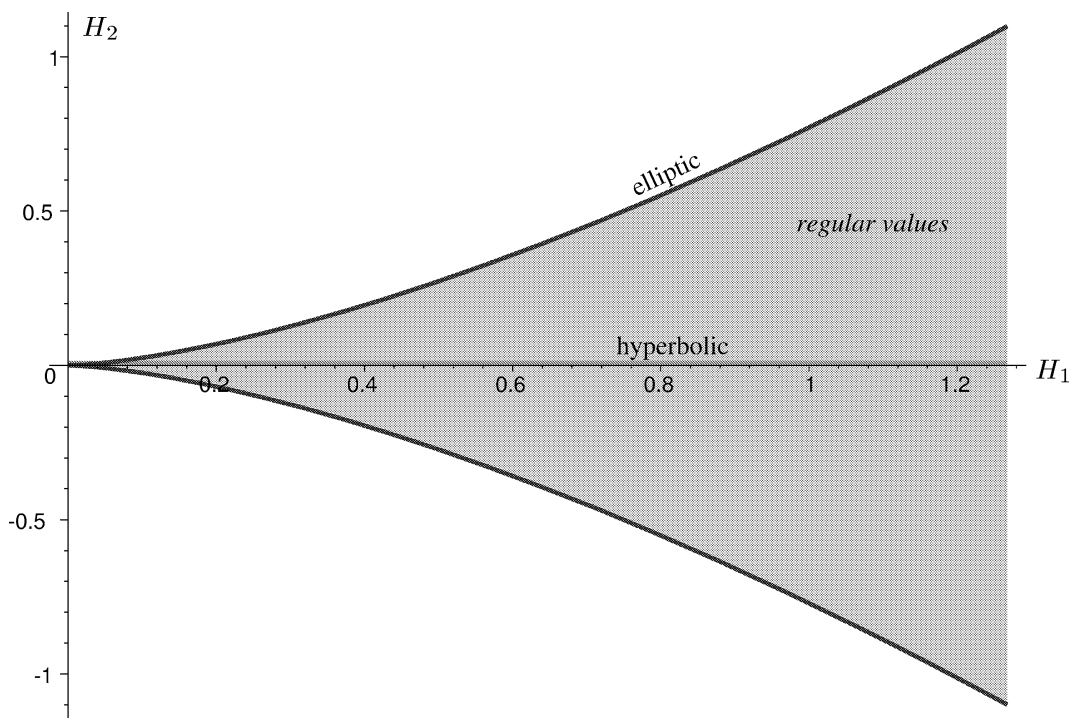

Fig. 16. Image of the momentum map for the $1: 2$ resonance. 


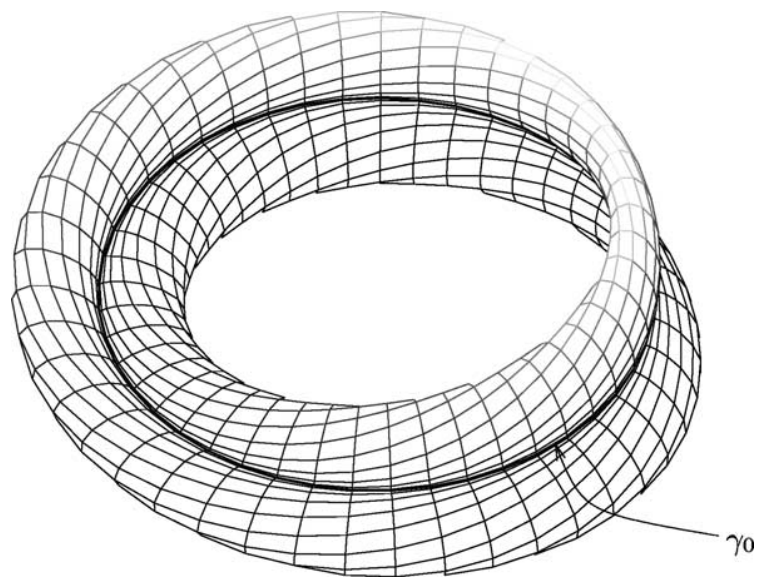

Fig. 17. The manifold $\Lambda_{o}$.

$$
\begin{aligned}
& \widehat{H}_{1}=\frac{1}{2}\left(-h^{2} \frac{\partial^{2}}{\partial x_{1}^{2}}+x_{1}^{2}\right)+\left(-h^{2} \frac{\partial^{2}}{\partial x_{2}^{2}}+x_{2}^{2}\right) \\
& \widehat{H}_{2}=x_{2}\left(h^{2} \frac{\partial^{2}}{\partial x_{1}^{2}}+x_{1}^{2}\right)-h^{2} \frac{\partial}{\partial x_{2}}\left(2 x_{1} \frac{\partial}{\partial x_{1}}+1\right) .
\end{aligned}
$$

\subsubsection{Classical description}

Here we are interested in the singular Lagrangian leaf $\Lambda_{o}$ defined by $H_{1}=1, H_{2}=0$. The singular part of $\Lambda_{o}$ is the closed trajectory $\gamma_{0}=\left\{z_{1}=0\right\} \cap\left\{\left|z_{2}\right|=1\right\}$. From its defining equations, it is easy to find a parameterisation that shows that $\Lambda_{o}$ is a Lagrangian immersion of a Klein bottle $\mathbb{K}$ with $\gamma_{0}$ as a double loop:

$$
\Phi: \mathbb{K} \ni(\theta, \varphi) \mapsto\left(\sqrt{2} \mathrm{e}^{i \theta} \sin \varphi,-i \mathrm{e}^{2 i \theta} \cos \varphi\right) \in \Lambda_{o}
$$

where $\mathbb{K}$ is the quotient of $\mathbb{T}_{(\theta, \varphi)}^{2}=\mathbb{R}^{2} / \mathbb{Z}^{2}$ by the equivalence relation

$$
(\theta+\pi,-\varphi) \sim(\theta, \varphi)
$$

A fundamental domain $D$ is given by $D=\{(\theta, \varphi) \mid 0 \leqslant \theta \leqslant \pi,-\pi \leqslant \varphi \leqslant \pi\}$. The singular line $\gamma_{0}$ corresponds to $\{\varphi=0\} \cup\{\varphi= \pm \pi\}$ and we have there the identifications

$$
\Phi(\theta, 0)=\Phi(\theta+\pi / 2, \pm \pi) .
$$

The graph

$$
G=\square
$$

corresponding to $\Lambda_{o}$ has just one vertex $\gamma_{0}$ and one edge.

Remark 3.1. - Although we do not really need it, it can be helpful to have a representation of the reduced phase space $W=H_{1}^{-1}(1) / S^{1}$ - where the $S^{1}$-action is the flow of the harmonic oscillator $H_{1}$. Using an a priori argument, one can show that $W$ is a 2 -sphere with a conical singular point; however, one can find an explicit equation for $W$. The algebra of $S^{1}$-invariant 


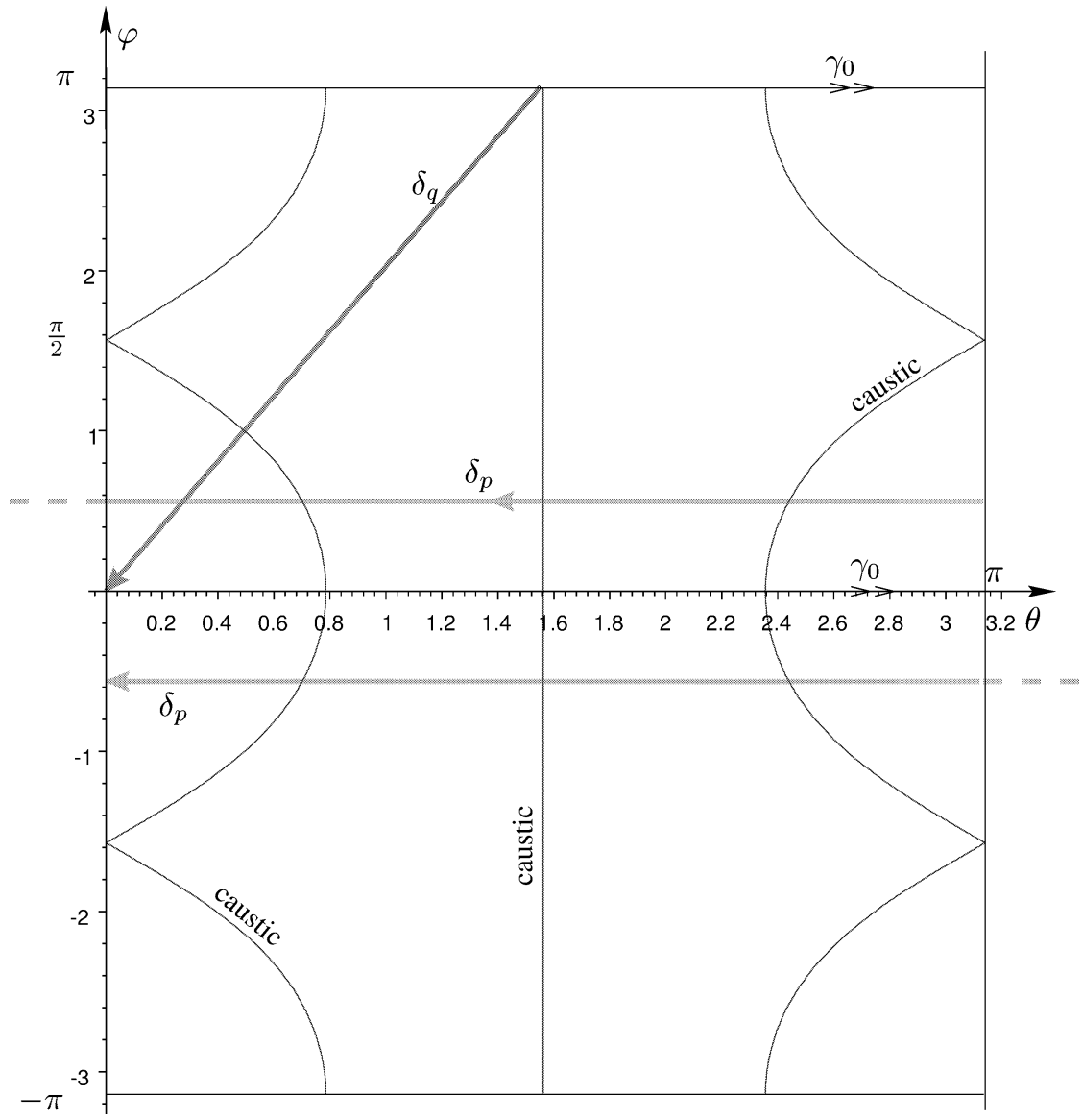

Fig. 18. Parameters set for $\Lambda_{o}$.

polynomials - that is, those that commute with $H_{1}$ - is generated by

$$
\pi_{1}=\left|z_{1}\right|^{2}, \quad \pi_{2}=\left|z_{2}\right|^{2}, \quad \pi_{3}=\mathfrak{R}\left(z_{1}^{2} \bar{z}_{2}\right), \quad \pi_{4}=\mathfrak{I}\left(z_{1}^{2} \bar{z}_{2}\right),
$$

which are subject to the relation $\pi_{3}^{2}+\pi_{4}^{2}=\pi_{1}^{2} \pi_{2}$. One can show (see the book [3]) that this relation together with $\pi_{1} \geqslant 0$ and $\pi_{2} \geqslant 0$ define the orbit space $T^{*} \mathbb{R}^{2} / S^{1}$ in terms of the variables $\pi_{j}$. The energy level set is the section $\left\{\pi_{1} \geqslant 0\right\} \cap\left\{\pi_{2} \geqslant 0\right\}$ of the 3-dimensional hyperplane $\pi_{1}+2 \pi_{2}=1$. Therefore, $W$ is defined in the space $\mathbb{R}^{3}=\left(\pi_{1}, \pi_{4}, \pi_{3}\right)$ by the equation

$$
\pi_{3}^{2}=\pi_{1}^{2}\left(1-\pi_{1}\right) / 2-\pi_{4}^{2}, \quad \text { with } \pi_{1} \in[0,1]
$$

$W$ is a surface of revolution around the $\pi_{1}$-axis, homeomorphic to a 2 -sphere, with a conical singularity at the origin. Note that $H_{2}=\pi_{3}$ so that the restriction $\left(H_{2}\right)_{\uparrow W}$ is just the height function (see Fig. 19) and is a Morse function on $W \backslash\{0\}$. The manifold $\Lambda_{o}$ reduces to the singular equator $\pi_{3}=0$. 




Fig. 19. The singular reduced phase space $W$. Here the function $\left(H_{2}\right)_{\uparrow W}$ is equal to the $\pi_{3}$ coordinate.

\subsubsection{Semiclassical computations}

We consider the solutions of the system

$$
\left(\widehat{H}_{1}-1\right) u=0, \quad\left(\widehat{H}_{2}-e h\right) u=0,
$$

for bounded $e$. The microsupport of the solutions is $\Lambda_{o}$.

If we denote by $\mathcal{X}_{j}$ the Hamiltonian vector fields of $H_{j}$ we get on $\Lambda_{o}$ :

$$
\mathcal{X}_{1}=-\frac{\partial}{\partial \theta}, \quad \mathcal{X}_{2}=-2 \sin \varphi \frac{\partial}{\partial \varphi}
$$

which leads to the following sub-principal form $\kappa$ for $\widehat{F}=\left(\widehat{H}_{1}, \widehat{H}_{2}-e h\right)$ :

$$
\kappa=\frac{-e}{2 \sin \varphi} d \varphi
$$

Note also that, since the flow of $\mathcal{X}_{1}$ is $2 \pi$-periodic outside $\gamma_{0}$, we have $\mathcal{X}_{p}=\mathcal{X}_{1}$. The canonical 1 -form $\alpha=\xi_{1} d x_{1}+\xi_{2} d x_{2}$ is given by:

$$
\alpha=-2\left(\sin ^{2} \theta \sin ^{2} \varphi+\cos ^{2} 2 \theta \cos ^{2} \varphi\right) d \theta+\frac{1}{2}(\sin 2 \theta(1+\cos 2 \theta) \sin 2 \varphi) d \varphi .
$$

Finally, the caustic set $C$ of $\Lambda_{o}$ is given by

$$
C=\{\cos \theta=0\} \cup\left\{\tan ^{2} \theta=\cos ^{2} \varphi\right\} .
$$

In order to compute the quantisation rules, let us introduce the following loops on $\Lambda_{o}$ :

$$
\begin{aligned}
& \delta_{p}(\pi-s)=\Phi\left(-s, \pm \varphi_{0}\right), \quad s \in[0, \pi] \quad \text { for some } \varphi_{0} \neq 0(\pi), \\
& \delta_{q}\left(\frac{\pi}{2}-s\right)=\Phi(-s,-2 s), \quad s \in[0, \pi / 2] .
\end{aligned}
$$

$\delta_{p}$ is an oriented $S^{1}$-orbit, and $\delta_{q}$ is a loop which is everywhere transversal to the $S^{1}$-action and oriented according to the flow of $\mathcal{X}_{2}$. 
The first quantisation condition:

$$
\frac{1}{2 \pi} \int_{\delta_{p}} \alpha+h \mu\left(\delta_{p}\right) / 4 \in h \mathbb{Z}+\mathrm{O}\left(h^{2}\right)
$$

is actually exact since $\widehat{H}_{1}$ is a harmonic oscillator, and therefore reads:

$$
1-\frac{6}{4} h=h n \quad \text { or } \quad h=\frac{1}{n+3 / 2}
$$

Because of the homogeneity property, we choose here to view this condition as a discrete quantisation of $h$ (see Remark 2.2).

Assuming now that (57) holds, we can compute the semiclassical invariants associated to $\delta_{q}$ :

The action integral is easily computed to be

$$
\int_{\delta_{q}} \alpha=\frac{\pi}{2},
$$

and the invariant $\varepsilon$ given by (12) is equal to $e / 2$. The sub-principal action $I_{\delta_{q}}$ of Definition 2.2 is given by

$$
I_{\delta_{q}}:=\int_{\delta_{q}} \tilde{\kappa}=3 \varepsilon \ln 2 .
$$

Finally, we can show by slightly shifting $\delta_{q}$ to the right (in the $\theta$ direction) that its Maslov index is -2 . Moreover $\delta_{q}$ turns around $\gamma_{0}$ in the direct sense, hence the regularised Maslov index is $-2+\left(\frac{1}{2}+n\right)$.

We can now write down the second quantisation condition: $\mathcal{C}_{0}=\operatorname{hol}\left(\delta_{q}\right)$, which reads:

$$
\begin{aligned}
& \mathrm{e}^{-i \frac{\pi}{4}-i n \frac{\pi}{2}}\left(1+i(-1)^{n} \mathrm{e}^{-\varepsilon \pi}\right) \Gamma\left(\frac{1}{2}+i \varepsilon\right) \mathrm{e}^{\varepsilon\left(\frac{\pi}{2}+i \ln h\right)} \\
& =\mathrm{e}^{i\left(\frac{\pi}{2 h}+3 \varepsilon \ln 2+\frac{\pi}{2}\left(-2+\left(\frac{1}{2}+n\right)\right)+\mathrm{O}(h)\right)} .
\end{aligned}
$$

Using (57) and $\varepsilon=e / 2$, we obtain the equation in $e$ and $n$ :

$$
\left(1+i(-1)^{n} \mathrm{e}^{-\frac{e \pi}{2}}\right) \Gamma\left(\frac{1}{2}+\frac{i e}{2}\right) \mathrm{e}^{\frac{e}{2}\left(\frac{\pi}{2}-i \ln (n+3 / 2)\right)}=\mathrm{e}^{i\left(\frac{3}{2} e \ln 2-\frac{\pi}{2} n+\frac{\pi}{4}+\mathrm{O}(h)\right)} .
$$

Remark 3.2. - The semiclassical invariants were computed explicitly; this is related to the fact that $\Lambda_{o}$ admits a parameterisation as a "rational" variety. Somewhat paradoxically, it would be more technical to compute the WKB invariants attached to regular tori, since no rational parameterisation of these tori exists. On the other hand, the regular invariants can be asymptotically recovered from the singular ones using Stirling's formula (35).

Remark 3.3. - The obtained formula (60) yields easily the fact that the level spacings for the eigenvalues in a region of bounded $e$ are of order $\mathrm{O}(1 / n \ln n)=\mathrm{O}(h / \ln h)$ - while they are of order $\mathrm{O}(h)$ in a regular region. Moreover, the precise shape of the spacing function is readily derived and involves the log-derivative of the Gamma function. 


\subsubsection{Matrix form for $\widehat{H}_{2}$}

The goal here is to study the restriction of $\widehat{H}_{2}$ to the eigenspace $\mathfrak{E}_{n}$ of $\widehat{H}_{1}$ corresponding to the quantum number $n$ (i.e. to the eigenvalue $h(n+3 / 2)$ ).

In analogy with formulae (52), the operators (49) and (50) can be written

$$
\begin{aligned}
& \widehat{H}_{1}=h\left(a_{1}(h) b_{1}(h)+2 a_{2}(h) b_{2}(h)-\frac{3}{2}\right), \\
& \widehat{H}_{2}=\sqrt{2} h^{3 / 2}\left(a_{2}(h) b_{1}(h)^{2}+a_{1}(h)^{2} b_{2}(h)\right),
\end{aligned}
$$

with $a_{1}(h)=(2 h)^{-1 / 2}\left(h \frac{\partial}{\partial x}+x\right)$ and $b_{1}(h)=a_{1}(h)^{*}=(2 h)^{-1 / 2}\left(-h \frac{\partial}{\partial x}+x\right)$ (and similarly for $a_{2}(h)$ and $b_{2}(h)$ with the variable $\left.y\right)$. Using the unitary transform in $L^{2}\left(\mathbb{R}^{2}\right)$

$$
f(x) \rightarrow \sqrt{h} f(\sqrt{h} x),
$$

the operators $\widehat{H}_{1}$ and $\widehat{H}_{2}$ are transformed into those given by Eqs. (61) and (62) with $a_{j}(h)$ and $b_{j}(h)$ replaced by $a_{j}:=a_{j}(1)$ and $b_{j}:=b_{j}(1)$. Note that this shows that the homogeneity argument used for the classical analysis has an analogue in the quantum setting: if we know the spectrum for some value of $h>0$, then the spectrum for any other value of $h$ immediately follows.

Now, using the Bargmann representation, we identify $a_{j}$ (respectively $b_{j}$ ) with the operator $\frac{\partial}{\partial z_{j}}$ (respectively $z_{j}$ ), and let them act on the monomials $\frac{z_{1}^{k} z_{2}^{\ell}}{k ! \ell !}$ which form a Hilbert basis of eigenvectors of $\widehat{H}_{1}$ (corresponding to the eigenvalues $E_{1}=h(k+2 \ell+3 / 2)$ ). Then it is easy to find the matrix of $\widehat{H}_{2}$ in this basis of $\mathfrak{E}_{n}(n=k+2 \ell)$ :

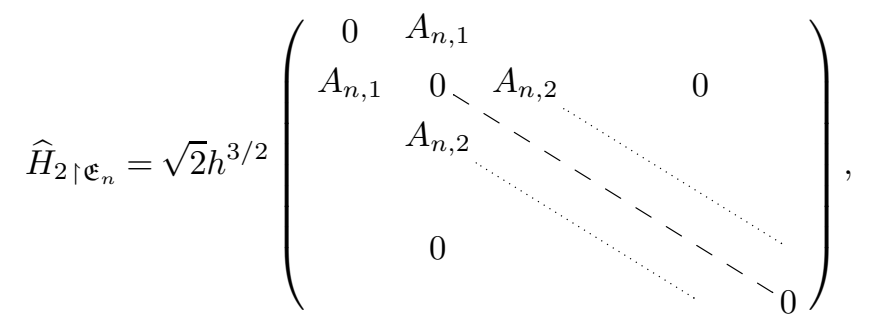

$$
\begin{aligned}
& \text { with } A_{n, \ell}=\sqrt{\ell(n-2 \ell+1)(n-2 \ell+2)}, \quad \ell=1,2, \ldots, E\left[\frac{n}{2}\right] .
\end{aligned}
$$

\subsubsection{Numerical computations}

Since $h$ is of order $1 / n$, one sees that the coefficients of (63) are bounded as $n \rightarrow \infty$. Moreover, since no coefficient $A_{n, \ell}$ vanishes, the spectrum is simple. For these reasons, it is reasonable to expect a good accuracy of numerically computed eigenvalues. The resulting spectrum will be called the "quantum" spectrum.

On the other hand, numerically solving Eq. (60) in the variable $e-$ assuming that he remains in the bounds of the momentum map - yields the so-called "semiclassical" spectrum for $E_{1}=1$ and $h=(n+3 / 2)^{-1}$. If we wish now to fix $h$ and compute the rest of the joint spectrum, the same formulae (58) and (59) can be used if one lets $\varepsilon=\frac{e}{2 \sqrt{E_{1}}}$ and replaces $h$ by $\tilde{h}=h / E_{1}$.

The results are displayed in the following figures. In Fig. 20 we have superposed the quantum and the semiclassical joint spectra. The differences are hardly noticeable (they are theoretically 


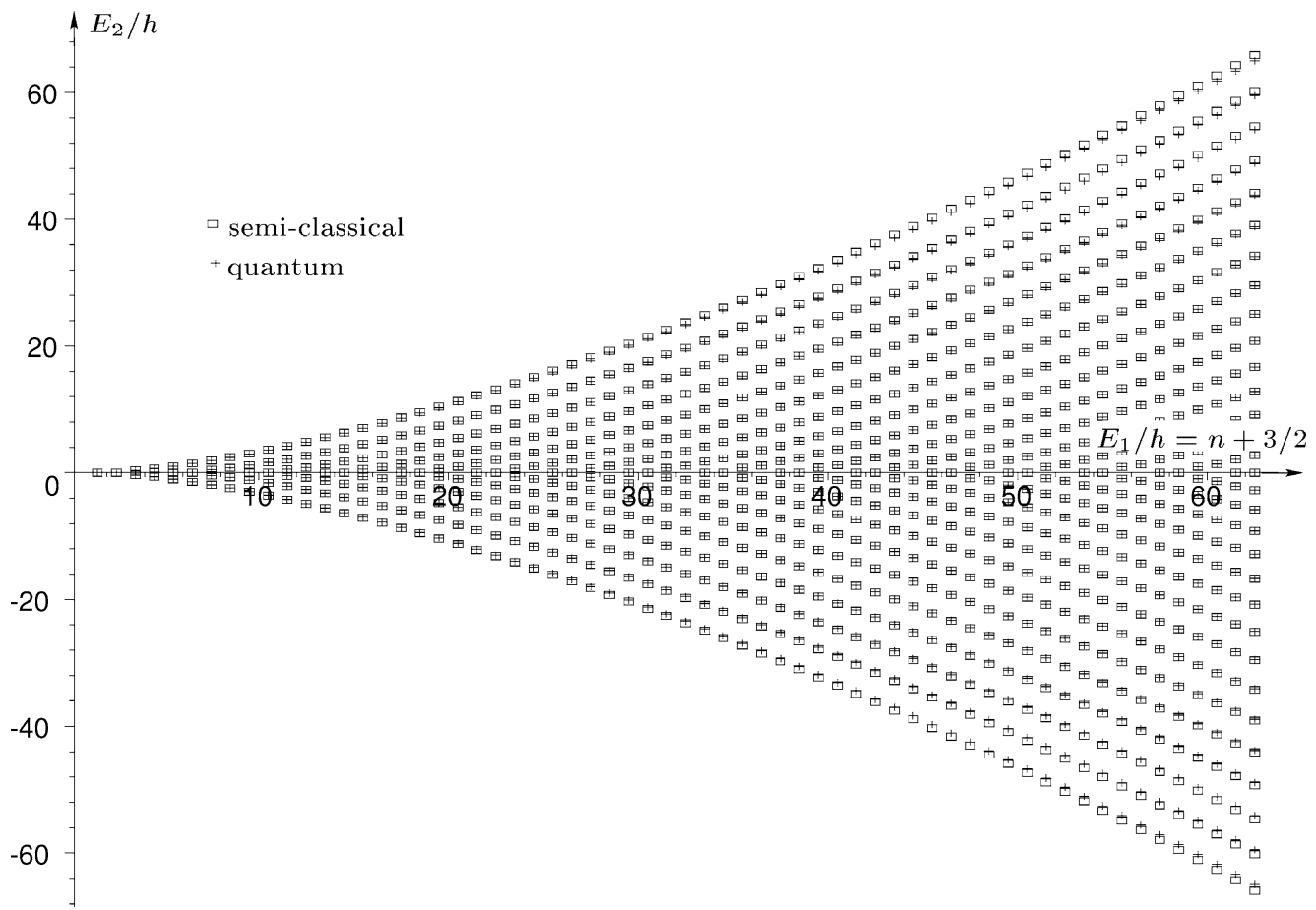

Fig. 20. A comparison between semiclassical and quantum results. Here $h=2 / 63$ (so that $E_{1}=1$ corresponds to $n=30$ ).

of order $h^{1}$ - which means $h^{2}$ for the unscaled spectrum - and experimentally much better at the critical value $H_{2}=0$ ), even for a very large $h$ and very small $E_{1}$ 's - both of these conditions are supposed to reach the limitations of our analysis.

In the other Figs. 21 and 22 we focus on one spectrum (here at $E_{1}=1$ ) around the critical value $E_{2}=0$ - which is the most interesting feature.

\subsection{Schrödinger operators on $S^{2}$}

\subsubsection{Setting of the problem}

We consider now the operator $\widehat{H}=\Delta+V$ where $\Delta$ is the canonical Laplacian on $S^{2}=\left\{(x, y, z) \in \mathbb{R}^{3} \mid x^{2}+y^{2}+z^{2}=1\right\}$ whose spectral theory is given by the spherical harmonics and $V: S^{2} \rightarrow \mathbb{R}$ is a smooth potential. We introduce the pseudodifferential operator $\widehat{H}_{2}$ on $S^{2}$ which is obtained by averaging $V$ using the $2 \pi$-periodic quantum unitary flow $U(t)=\exp (i t \sqrt{\Delta+1 / 4})$ :

DEFINITION 3.1.-

$$
\widehat{H}_{2}=\frac{1}{2 \pi} \int_{0}^{2 \pi} U(-t) V U(t) d t .
$$

The following results have been obtained by Weinstein and Guillemin (see $[29,15,17,16]$ and also [6,7]): 


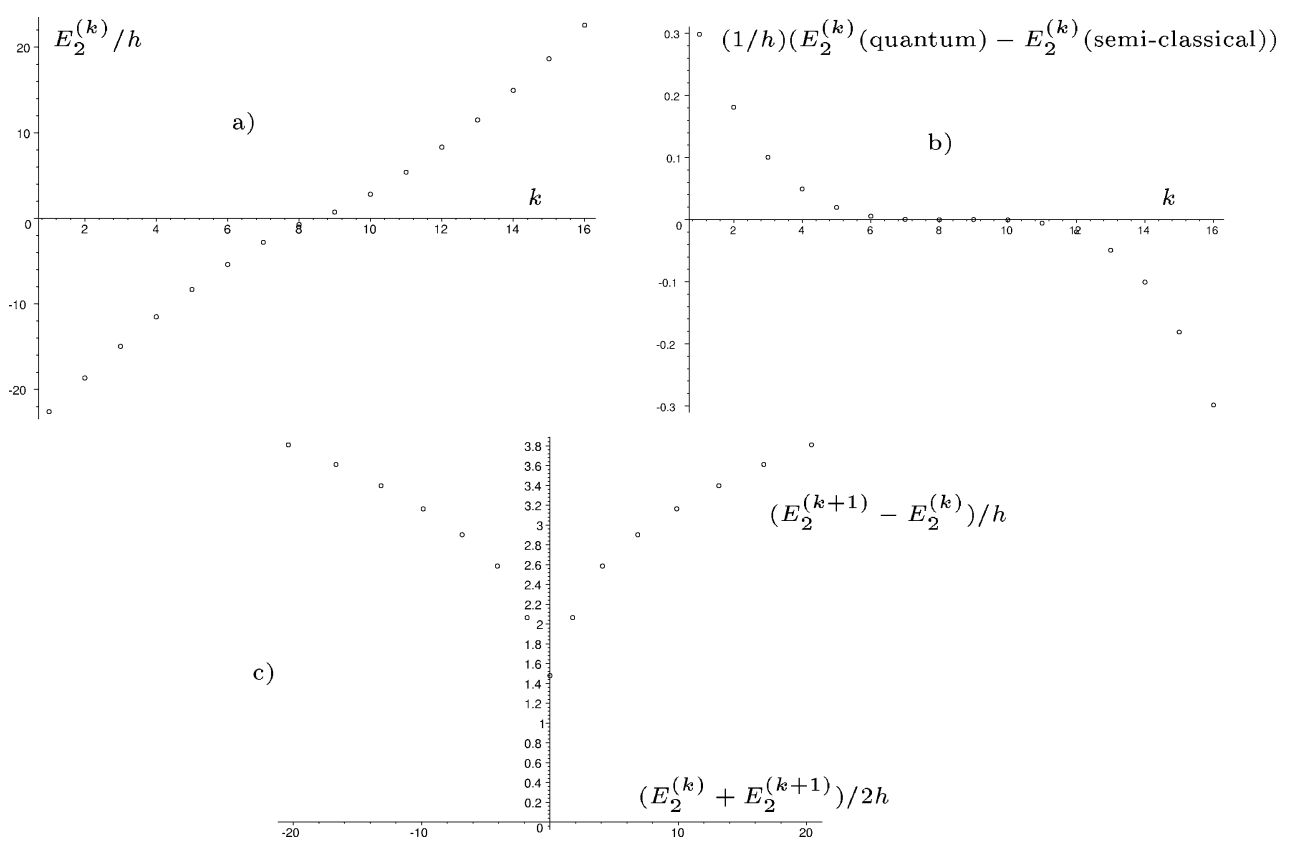

Fig. 21. The spectrum at $E_{1}=1, n=30$ (quantum and semiclassical are indistinguishable). (a) The spectrum sorted in increasing order and displayed versus the eigenvalue number. (b) The difference "quantum-semiclassical". (c) The energy spacings.
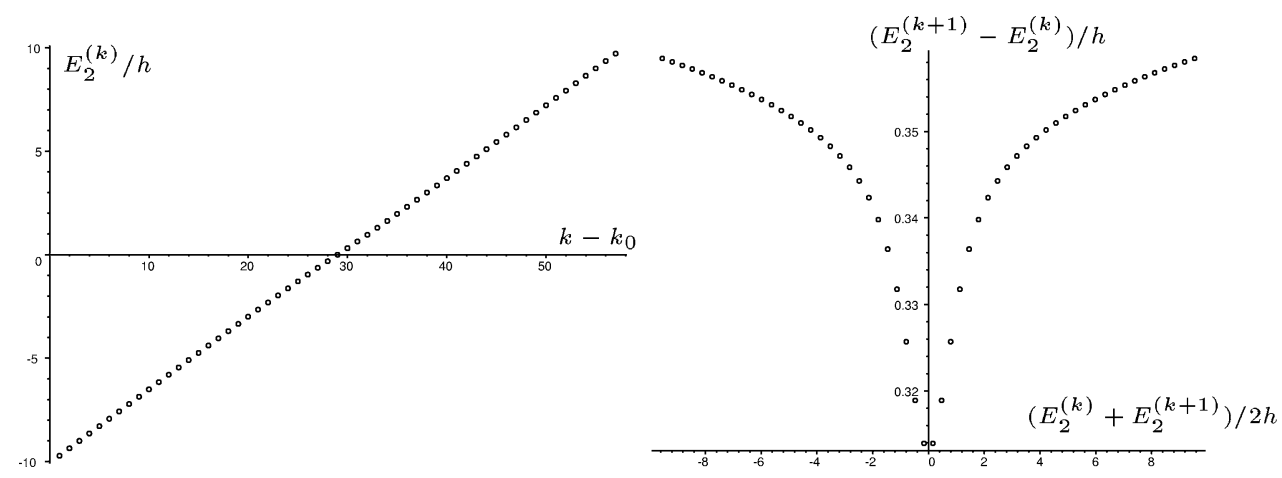

Fig. 22. Contrary to the numerical diagonalisation of the matrix (63), the semiclassical formula allows very small values of $h-$ and the results are supposedly even more accurate. Here is displayed a window of size $[-10 h, 10 h]$ for the spectrum and the corresponding eigenvalue spacings, where we have let $E_{1}=1$ and $n=10^{15}$ ( $h \simeq 10^{-15}$ !! of course we have not tried the matrix diagonalisation !).

\section{THEOREM 3.2.-}

- $\widehat{H}_{2}$ commute with $\Delta$.

- $\widehat{H}_{2}$ is a PDO of order 0 whose principal symbol is the Radon transform of $V$ :

$$
H_{2}(z)=\frac{1}{2 \pi} \int_{0}^{2 \pi} V\left(\varphi_{t}(z)\right) d t,
$$


where $\varphi_{t}$ is the geodesic flow with unit speed. The sub-principal symbol of $\widehat{H}_{2}$ vanishes.

- There exists an unitary FIO $\Omega$ such that

$$
\Omega^{-1} \widehat{H} \Omega=\Delta+\widehat{H}_{2}+R
$$

where $R$ is a PDO of order -2 .

$$
\widehat{H}_{2}=\bigoplus_{l=0}^{\infty} \Pi_{l} V \Pi_{l}
$$

where the $\Pi_{l}$ 's are the orthogonal projections on the spaces $\mathcal{H}_{l}$ of spherical harmonics of degree l.

Proofs can be found in [15] and [17].

In such a way, we get a quantum integrable system $\widehat{H}_{1}=h^{2} \Delta, \widehat{H}_{2}$. The spectrum of $\widehat{H}$ is related to the joint spectrum

$$
\left(h^{2} l(l+1), \mu_{l, m}\right), \quad l=0, \ldots, \infty,-l \leqslant m \leqslant l
$$

of $\left(\widehat{H}_{1}, \widehat{H}_{2}\right)$ by $\lambda_{l, m}=l(l+1)+\mu_{l, m}+\mathrm{O}\left(l^{-2}\right)$ and high energy asymptotics $(l \rightarrow \infty)$ correspond in the usual way to semiclassical asymptotics $h^{2} . l(l+1)=1, h \rightarrow 0$. We will study the system:

$$
\widehat{H}_{1} \varphi=h^{2} l(l+1) \varphi=\varphi, \quad \widehat{H}_{2} \varphi=e h \varphi,
$$

assuming that 0 is a critical value of saddle type of $H_{2}$.

The Radon transform $H_{2}$ of $V$ is a function on the manifold Geod of oriented closed geodesics of $S^{2}$. Geod is a global Poincaré section for $H_{1}$ and can be identified with $S^{2} \subset \mathbb{R}_{X, Y, Z}^{3}$ by associating to the circle $t \rightarrow \gamma(t)=u \cos t+v \sin t$ the unit vector $u \wedge v$. Then reversing the orientation of $\gamma$ corresponds to antipodal symmetry $\sigma$ on $G e o d=S^{2}$ and $H_{2}$ is even with respect to that symmetry. We can then interpret $H_{2}$ as a function on the projective plane. This fact implies that, if $H_{2}$ is a Morse-Bott function, it cannot have only local maxima and minima: it has always saddle points for which our analysis is needed.

We will from now assume that we are in the simplest situation where $H_{2}:$ Geod $\rightarrow \mathbb{R}$ has only 2 maxima, 2 minima and 2 non-degenerate saddle points. The singular manifold $\Lambda_{0}$ is then the union of 2 tori which intersect along 2 circles. The projection of $\Lambda_{0}$ on Geod $=S^{2}$, i.e. the reduction of $\Lambda_{0}$, is the union of 2 circles which are invariant by $\sigma$ and which intersect at 2 antipodal points.

\subsubsection{Semiclassical computations}

We will complete the computations in the simplest case where $V$ itself is a generic harmonic polynomial of degree 2 . Using $S O(3)$ invariance, we have only to consider the 2-parameter family given by

$$
V_{a, b, c}(x, y, z)=2\left(a x^{2}+b y^{2}+c z^{2}\right),
$$

with $a+b+c=0$ and $a<c<b$.

Because the Radon transform commutes with the $S O(3)$ action, by Schur's lemma, $\mathrm{H}_{2}$ itself is an harmonic polynomial of degree 2 on Geod which is given by

$$
H_{2}=a X^{2}+b Y^{2}+c Z^{2} .
$$



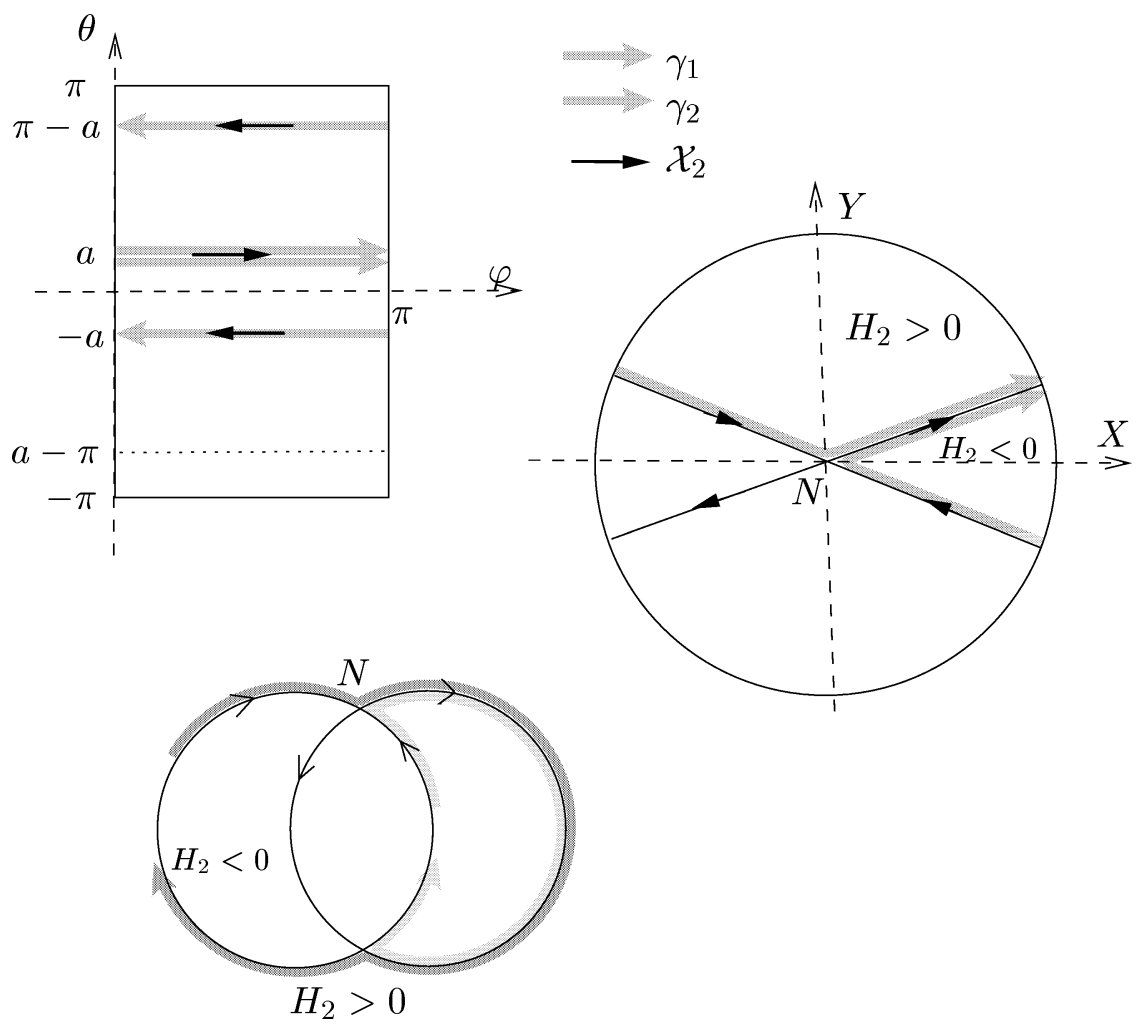

Fig. 23. $G$ in Geod.

The critical values of $H_{2}$ are $a<c<b$ and $H_{2}-c=B^{2} Y^{2}-A^{2} X^{2}$ with $A=\sqrt{c-a}$ and $B=\sqrt{b-c}$. It is clear that the singular value $(1, c)$ of $\left(H_{1}, H_{2}\right)$ is of hyperbolic type, so that we can apply the previous tools.

Let us denote by $G$ the projection of $\Lambda_{0}$ on Geod by the map $\pi$ which associates to a point $m \in U^{\star} S^{2}$ the geodesic to which $m$ belongs; $G$ is the graph introduced in the general situation and is the union of the two circles $C_{\tau}=\{A X=\tau B Y, \tau= \pm 1\}$. We can compute the projections on $G$ of the vector field $\mathcal{X}_{2}$ and of the sub-principal form $\kappa$ on $\Lambda_{0}$ (because $\kappa\left(\mathcal{X}_{1}\right)=0$ ). We will use spherical coordinates $(\theta, \varphi), 0 \leqslant \theta \leqslant 2 \pi, 0 \leqslant \varphi \leqslant \pi$, on Geod:

$$
X=\sin \varphi \cos \theta, \quad Y=\sin \varphi \sin \theta, \quad Z=\cos \varphi .
$$

The symplectic form on Geod is $S O(3)$ invariant and of total area $4 \pi$. We will assume that:

$$
\omega=\sin \varphi d \theta \wedge d \varphi .
$$

By direct computation we get on $C_{\tau}$ :

$$
\mathcal{X}_{2}=2 \tau A B \sin \varphi \frac{\partial}{\partial \varphi}
$$

and

$$
\kappa=\frac{\tau e}{2 A B \sin \varphi} d \varphi .
$$


Let us denote by $\gamma_{j}, j=1, \ldots, 4$, the four cycles of $G$ oriented by $\mathcal{X}_{2}$, consisting of the union of one arc of $C_{1}$ and one arc of $C_{-1}$ and bounding a topological disk in Geod. $\gamma_{1}$ and $\gamma_{2}$ are defined on Fig. 23 and $\gamma_{3}=\sigma\left(\gamma_{1}\right), \gamma_{4}=\sigma\left(\gamma_{2}\right)$. It is then easy to check using the explicit formula for the integral $\int d \varphi / \sin \varphi$ that

$$
\forall j=1, \ldots, 4, \quad I_{\gamma_{j}}=\frac{e}{A B} \log \frac{8 A B}{A^{2}+B^{2}} .
$$

Moreover, we find easily, using formula (12), that

$$
\varepsilon_{2}=\frac{e}{2 A B} .
$$

We put $\alpha=\operatorname{atan} \frac{A}{B}$ and $\beta=\operatorname{atan} \frac{B}{A}=\frac{\pi}{2}-\alpha$. Action integrals are $A_{1}=A_{3}=-4 \beta$ and $A_{2}=A_{4}=4 \alpha$.

Let $\tau_{j}=\operatorname{hol}\left(\gamma_{j}\right)$. We observe first, using the fact that $h\left(l+\frac{1}{2}\right)=1$, the relations $\tau_{1}=\tau_{3}$ and $\tau_{2}=\tau_{4}$ (modulo $\mathrm{O}(h)$ ). We get

$$
\tau_{1}=\mathrm{e}^{i(-(4 l+2) \beta+I+\pi+\mathrm{O}(h))}
$$

and

$$
\tau_{2}=\mathrm{e}^{i((4 l+2) \alpha+I+\mathrm{O}(h))} .
$$

It follows that $H:=\tau_{1}=\tau_{2}=\tau_{3}=\tau_{4}$. It would be nice to prove that this relation holds $\bmod \mathrm{O}\left(h^{\infty}\right)$.

Using the computations of [10] p. 493 and putting $T=T(\varepsilon)$ with $\varepsilon=\varepsilon_{2}+\mathrm{O}(h)$ we get the following quantisation rule:

$$
\operatorname{det}\left(\operatorname{Id}-T\left(\begin{array}{ll}
0 & 1 \\
1 & 0
\end{array}\right) T\left(\begin{array}{cc}
0 & H^{-1} \\
H^{-1} & 0
\end{array}\right)\right)=0
$$

Putting

$$
T=\mathcal{E}\left(\begin{array}{cc}
1 & \omega \\
\omega & 1
\end{array}\right)
$$

we get:

$$
H=(1 \pm \omega)^{2} \mathcal{E}^{2}
$$

and the quantisation rule:

$$
\mathrm{e}^{i((4 l+2) \alpha+I+\mathrm{O}(h))}=\frac{1}{2 \pi} \Gamma\left(\frac{1}{2}+i \varepsilon\right)^{2} \mathrm{e}^{\varepsilon\left(\pi-2 i \log \left(l+\frac{1}{2}\right)\right)}\left(1 \pm i \mathrm{e}^{-\varepsilon \pi}\right)^{2},
$$

which has to be solved in $e$ where $e$ enters in $I$ and in $\varepsilon$.

Remark 3.4. - Because of the \pm sign in (60), the spectrum can be separated in two spectra. For each of these, the spacings of eigenvalues are easily computed as in Remark 3.3. Moreover, formula (60) shows that far from the critical value (i.e. $e \rightarrow \pm \infty$ ), the "+" and "-" eigenvalues associate in doublets, and that there is a universal transition happening when crossing the critical value $(e=0)$, where a doublet " ++ " becomes a doublet "- -+ ". The details for these formulae are similar to [9] and left to the reader. 


\subsubsection{Matrix form for $\widehat{H}_{2}$}

Since $V$ is a harmonic polynomial of degree 2, the Toeplitz operator $\widehat{H}_{2}$ is given by

$$
\widehat{H}_{2}=\Pi_{2} V \Pi_{2},
$$

where $V$ here is just the multiplication by $V$ and $\Pi_{2}$ is the orthogonal projector on the space $\mathcal{H}_{2}$ of spherical harmonics of degree 2 . We shall first determine the explicit formula for $\widehat{H}_{2}$ with a generic $V \in \mathcal{H}_{2}$ and then apply it to the specific form $V=2 a x^{2}+2 b y^{2}+2 c z^{2}$.

The spaces $\mathcal{H}_{l}$ are seen as the spaces of the irreducible representation of $\mathfrak{s o}(3)$ on $L^{2}\left(S^{2}\right)$, acting via the differential operators

$$
\begin{aligned}
L_{x} & =y \frac{\partial}{\partial z}-z \frac{\partial}{\partial y} \\
L_{y} & =z \frac{\partial}{\partial x}-x \frac{\partial}{\partial z} \\
L_{z} & =x \frac{\partial}{\partial y}-y \frac{\partial}{\partial x}
\end{aligned}
$$

which are subject to the relation

$$
\left[L_{x}, L_{y}\right]=L_{z} \quad(\text { and cyclic permutations of }(x, y, z))
$$

These operators commute with $\Delta$ and thus preserve $\mathcal{H}_{l}$. As usual (see e.g. [30]), we use the coordinates $\zeta=x+i y$ and $z$, and let

$$
\Omega_{ \pm}=i L_{x} \pm L_{y}
$$

so that $\Omega_{+}=-\bar{\zeta} \frac{\partial}{\partial z}+2 z \frac{\partial}{\partial \zeta}$ and $\Omega_{-}=\Omega_{+}^{*}=\zeta \frac{\partial}{\partial z}-2 z \frac{\partial}{\partial \bar{\zeta}}$. A natural basis of $\mathcal{H}_{l}$ is then the following:

$$
\mathfrak{B}_{l}=\left(\zeta^{l}, \Omega_{+} \zeta^{l}, \Omega_{+}^{2} \zeta^{l}, \ldots, \Omega_{+}^{m} \zeta^{l}, \ldots, \Omega_{+}^{2 l} \zeta^{l}\right) .
$$

We shall use the convenient equivalent representation given by the action of $\mathfrak{s u}(2)$ on the spaces $\mathcal{P}_{2 l}$ of homogeneous polynomials of degree $2 l$ in $\mathbb{C}^{2}=(\xi, \eta)$. Using the following identification

$$
\begin{aligned}
L_{x} & =\frac{1}{2 i}\left(\eta \frac{\partial}{\partial \xi}+\xi \frac{\partial}{\partial \eta}\right), \\
L_{y} & =\frac{1}{2}\left(-\eta \frac{\partial}{\partial \xi}+\xi \frac{\partial}{\partial \eta}\right), \\
L_{z} & =\frac{1}{2 i}\left(\xi \frac{\partial}{\partial \xi}-\eta \frac{\partial}{\partial \eta}\right),
\end{aligned}
$$

we get $\Omega_{+}=\xi \frac{\partial}{\partial \eta}$, and a natural basis of $\mathcal{P}_{2 l}$ is the following:

$$
\left(\eta^{2 l}, \Omega_{+} \eta^{2 l}, \Omega_{+}^{2} \eta^{2 l}, \ldots, \Omega_{+}^{m} \eta^{2 l}, \ldots, \Omega_{+}^{2 l} \eta^{2 l}\right)
$$

In the rest of the argument, this basis together with the basis (70) will be used to identify $\mathcal{H}_{l}$ and $\mathcal{P}_{2 l}$. With this identification, $V$ assumes the form:

$$
V=\frac{a-b}{2}\left(\eta^{4}+\xi^{4}\right)-3(a+b) \eta^{2} \xi^{2} .
$$


Up to a multiplicative constant (depending on $l$ ), there exists, for each $l$, a unique equivariant morphism $\Pi: \mathcal{H}_{2} \otimes \mathcal{H}_{l} \rightarrow \mathcal{H}_{l}$. Hence $\Pi$ is a multiple of $\mathcal{D}^{2}$, where

$$
\mathcal{D}=\frac{\partial}{\partial \eta} \otimes \frac{\partial}{\partial \xi}-\frac{\partial}{\partial \xi} \otimes \frac{\partial}{\partial \eta}
$$

(A subsequent composition by the multiplication $f \otimes g \rightarrow f g$ is always assumed.)

On the other hand, using the fact that every element of the form $f g, f \in \mathcal{H}_{2}$ and $g \in \mathcal{H}_{l}$ splits into

$$
f g=f_{l+2}+r^{2} f_{l}+r^{4} f_{l-2},
$$

where $r$ is the radial distance and $f_{j} \in \mathcal{H}_{j}$, one easily computes

$$
f_{l}=\Pi(f \otimes g)=\frac{1}{6+4 l}\left(\Delta_{\mathbb{R}^{3}}(f g)-\frac{r^{2}}{2(2 l-1)} \Delta_{\mathbb{R}^{3}}^{2}(f g)\right) .
$$

Testing this formula and $\mathcal{D}^{2}$ with, for instance, $f=x^{2}-z^{2}$ and $g=(x+i y)^{l}$, one gets

$$
\begin{aligned}
\boldsymbol{\Pi} & =-\frac{1}{6(2 l-1)(3+2 l)} \mathcal{D}^{2} \\
& =-\frac{1}{6(2 l-1)(3+2 l)}\left(\frac{\partial^{2}}{\partial \xi^{2}} \otimes \frac{\partial^{2}}{\partial \eta^{2}}-2 \frac{\partial^{2}}{\partial \xi \partial \eta} \otimes \frac{\partial^{2}}{\partial \xi \partial \eta}+\frac{\partial^{2}}{\partial \eta^{2}} \otimes \frac{\partial^{2}}{\partial \xi^{2}}\right) .
\end{aligned}
$$

Now it is easy to let this operator act on $V \mathcal{H}_{l}$, and one finally obtains the matrix representation of $\widehat{H}_{2}$ in the basis $\mathfrak{B}_{l}$

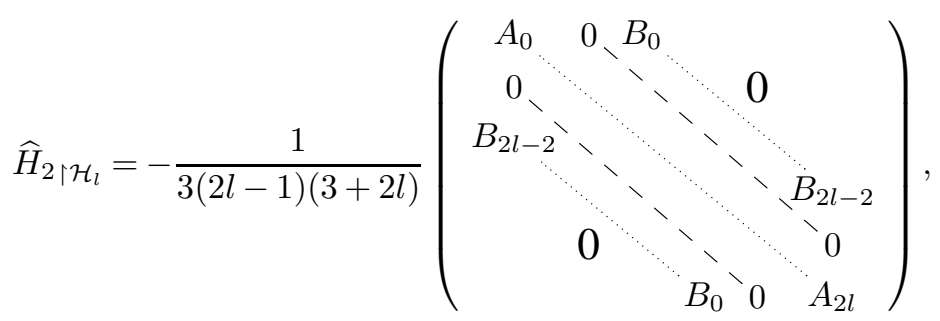

with

$$
\left\{\begin{array}{l}
A_{m}=6(a+b)(3 m(2 l-m)-l(2 l-1)), \\
B_{m}=3(a-b)(m+1)(m+2) .
\end{array}\right.
$$

Notice the symmetry of the matrix: $A_{m}=A_{2 l-m}$.

\subsubsection{Numerical computations}

As it is, the matrix (71) is very badly prepared for being numerically diagonalised. Indeed, the spectrum exhibits near degeneracies - as is expected from tunneling effects - and usual algorithms will rapidly fail as $l$ increases. Fortunately, there is an easy way to cope with this, for two commuting transformations can split the matrix: the projection onto the subspace spanned by the vectors from the basis (70) having even index, and the central symmetry $(m \rightarrow 2 l-m)$ of the matrix. We arrive at the following 4-blocks decomposition for the matrix $-3(2 l-1)(3+2 l) \widehat{H}_{2 \nmid \mathcal{H}_{l}}$, each block being a tridiagonal matrix:

- if $l=2 k+1$ is odd: 


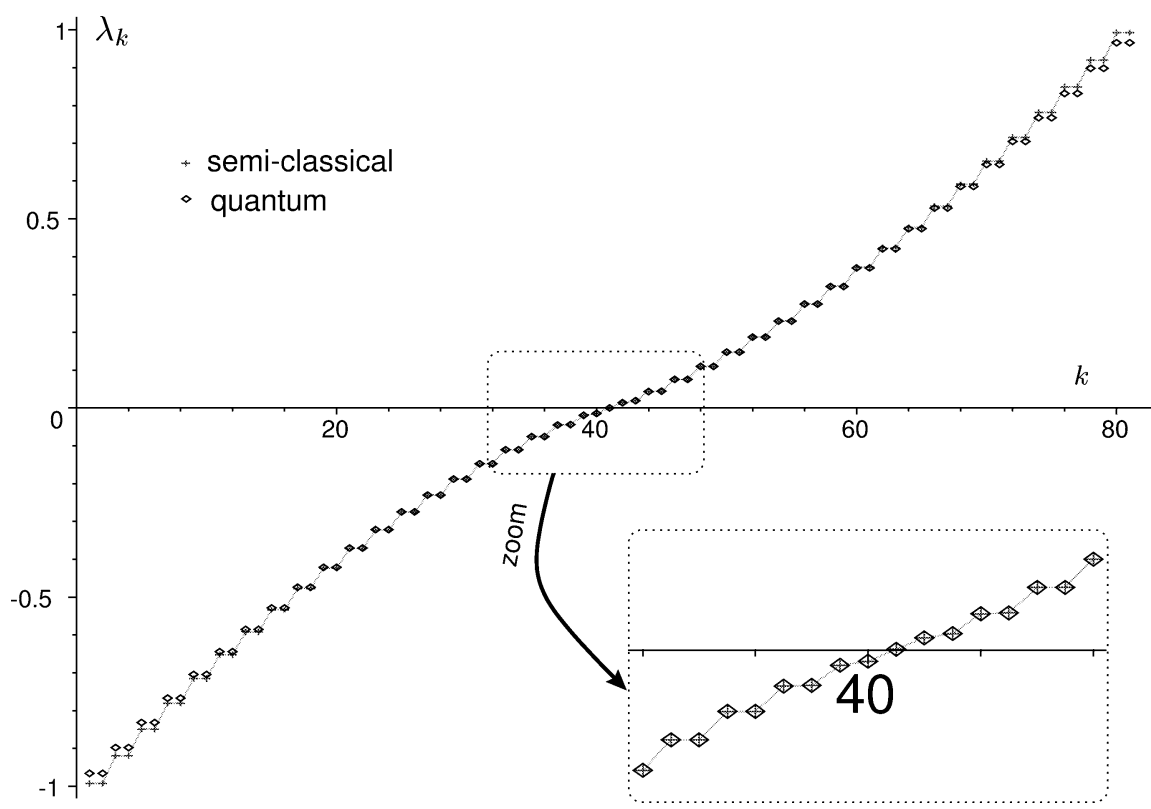

Fig. 24. A comparison between semiclassical and quantum computations. Here is displayed the spectrum of $\mathfrak{H}_{2} \uparrow \mathcal{H}_{l}$ in increasing order versus the eigenvalue number, for $l=40$ and the potential $V$ defined by $a=-1$ and $b=1$. The light crosses linked by line segments are the semiclassical computations while the quantum eigenvalues are the dark diamonds. We observe a very good accuracy (of mean order $\mathrm{O}(h)=\mathrm{O}(1 / l)$ predicted by the theory, but much better near the critical value). Notice also how the eigenvalue doublets reassociate when passing through the critical value.

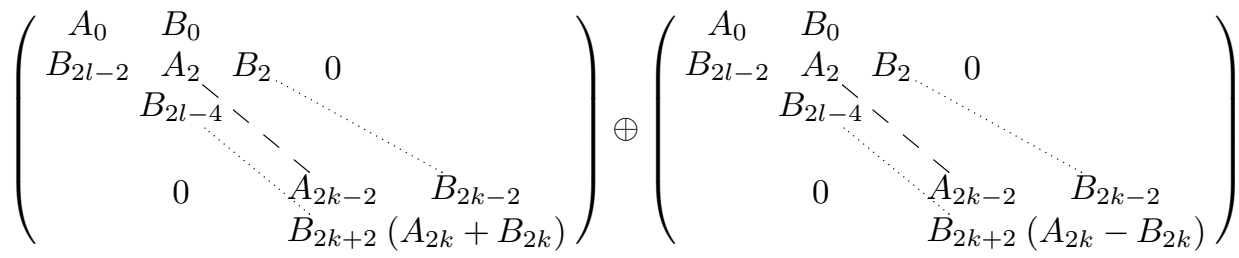



- if $l=2 k$ is even:

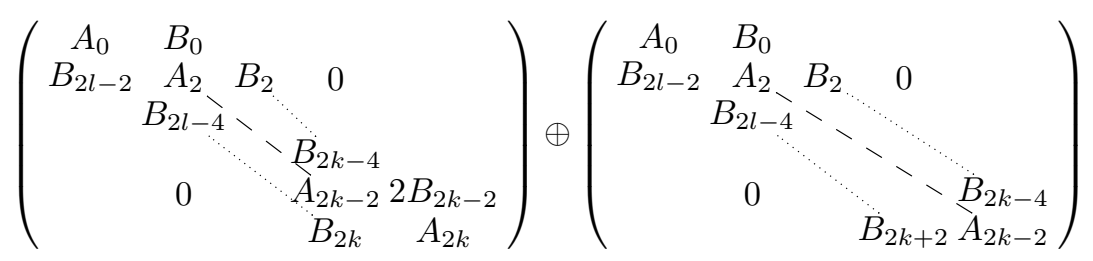




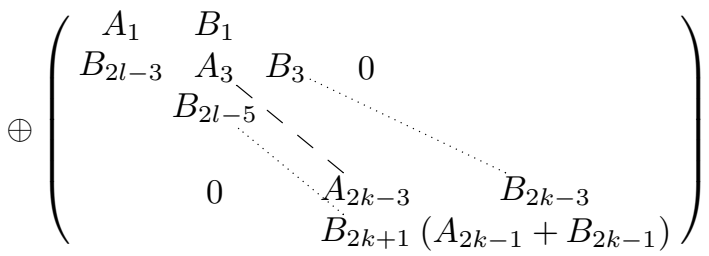

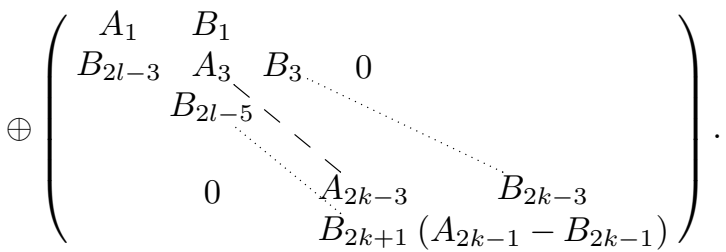

\section{REFERENCES}

[1] Ahlfors L., Sario S., Riemann Surfaces, Princeton University Press, 1960.

[2] Audin M., Courbes algébriques et systèmes intégrables: géodésiques des quadriques, Expositiones Math. 12 (1994) 193-226.

[3] Bates L., Cushman R., Global Aspects of Classical Integrable Systems, Birkhäuser, 1998.

[4] Bates S., Weinstein A., Lectures on the Geometry of Quantization, in: Berkeley Mathematics Lecture Notes, Vol. 8, AMS, 1997.

[5] ChILD M.S., Semiclassical Mechanics with Molecular Applications, Oxford University Press, 1991.

[6] COLIN DE VerdiÈre Y., Sur le spectre des opérateurs à bicaractéristiques toutes périodiques, Comment. Math. Helv. 54 (1979) 508-522.

[7] COLIN DE Verdière Y., Sur le spectre des opérateurs elliptiques à bicaractéristiques toutes périodiques, Math. Z. 171 (1980) 51-73.

[8] Colin de Verdière Y., PARISSE B., Équilibre instable en régime semi-classique I: Concentration microlocale, Comm. Partial Differential Equations 19 (9-10) (1994) 1535-1563.

[9] Colin de Verdière Y., PARISSE B., Équilibre instable en régime semi-classique II: Conditions de Bohr-Sommerfeld, Ann. Inst. H. Poincaré. Phys. Théor. 61 (3) (1994) 347-367.

[10] Colin de Verdière Y., Parisse B., Singular Bohr-Sommerfeld rules, Comm. Math. Phys. 205 (1999) 459-500.

[11] Colin de Verdière Y., Vey J., Le lemme de Morse isochore, Topology 18 (1979) 283-293.

[12] Darboux G., Théorie générale des surfaces, Chelsea, 1972.

[13] DuistermaAT J., Oscillatory integrals, Lagrange immersions and unfoldings of singularities, Comm. Pure Appl. Math. 27 (1974) 207-281.

[14] Fomenko A., Topological Classification of Integrable Systems, in: Advances in Soviet Mathematics, Vol. 6, AMS, 1991.

[15] GuilLemin V., Some spectral results for the Laplace operator with potential on the $n$-sphere, $A d v$. in Math. 27 (1978) 273-286.

[16] Guillemin V., Some spectral results on rank one symmetric spaces, Adv. in Math. 28 (1978) 129137.

[17] Guillemin V., Band asymptotics in two dimensions, Adv. in Math. 42 (1981) 248-282.

[18] Guillemin V., SchaefFer D., On a certain class of Fuchsian partial differential equations, Duke Math. J. 44 (1) (1977) 157-199.

[19] Hirzebruch F., Topological Methods in Algebraic Geometry, in: Grundlehren der math. W., Vol. 131, Springer, New York, 1966.

[20] Klingenberg W., Riemannian Geometry, de Gruyter, 1982.

[21] Moser J., Geometry of quadrics and spectral theory, in: The Chern Symposium, Springer, 1980, pp. 147-188. 
[22] NGUYÊn TIÊN Z., Singularities of integrable geodesic flows on multidimensional torus and sphere, J. Geom. Phys. 18 (1996) 147-162.

[23] NGUYÊN TIÊN Z., Symplectic topology of integrable hamiltonian systems, I: Arnold-Liouville with singularities, Compositio Math. 101 (1996) 179-215.

[24] Nguyên Tiên Z., Polyakova L., Selianova E., Topological classification of integrable geodesic flows on orientable two-dimensional manifolds, Funct. Anal. Appl. 27 (1993) 186-196.

[25] VŨ NGọc S, Formes normales semi-classiques des systèmes complètement intégrables au voisinage d'un point critique de l'application moment, Asympt. Analys. 24 (3,4) (2000) 319-342.

[26] Vũ Ngọc S., Sur le spectre des systèmes complètement intégrables semi-classiques avec singularités, Ph.D. thesis, Université Grenoble 1, 1998.

[27] VŨ NGọc S., Bohr-Sommerfeld conditions for integrable systems with critical manifolds of focusfocus type, Comm. Pure Appl. Math. 53 (2) (2000) 143-217.

[28] Weinstein A., Lectures on Symplectic Manifolds, in: Regional Conference Series in Mathematics, Vol. 29, AMS, 1976.

[29] Weinstein A., Asymptotics of eigenvalue clusters for the laplacian plus a potential, Duke Math. J. 44 (4) (1977) 883-892.

[30] WEYL H., The Theory of Groups and Quantum Mechanics, Dover, 1950, Translated from the (second) German edition.

(Manuscrit reçu le 22 février 2001; accepté, après révision, le 4 février 2002.)

Yves COLIN DE VERDiÈre

Institut Fourier,

Unité mixte de recherche CNRS-UJF 5582,

BP 74,

38402 Saint-Martin d'Hères cedex,

France

E-mail: yves.colin-de-verdiere@ujf-grenoble.fr

San VŨ NGọC

Institut Fourier,

Unité mixte de recherche CNRS-UJF 5582,

BP 74,

38402 Saint-Martin d'Hères cedex,

France

E-mail: san.vu-ngoc@ujf-grenoble.fr 